\title{
Integrando microestruturas de contágio econômico portfólios de crédito
}

Wellington Luiz Bogarim de Faria

TESE APRESENTADA

$\mathrm{AO}$

INSTITUTO DE MATEMÁTICA E ESTATÍSTICA

DA

UNIVERSIDADE DE SÃO PAULO

PARA

OBTENÇÃO DO TÍTULO DE DOUTOR

$\mathrm{EM}$

CIÊNCIAS

Área de Concentração: Matemática

Orientador: Prof. Dr. Pedro Paulo Serpa Schirmer

Durante a realização deste trabalho o autor recebeu auxílio financeiro do CNPQ

23 de outubro de 2007 


\section{Integrando microestruturas}

de

contágio econômico

em

portfólios de crédito

Tese apresentada ao

Instituto de Matemática e Estatística

da Universidade de São Paulo

Para obtenção do título de

Doutor em Ciências

área de concentração: Matemática

23 de outubro de 2007

Banca examinadora:

Pedro Paulo Serpa Schirmer (Orientador)- IME-USP

Henrique Von Dreifus-IME-USP

Gerson Francisco-IFT-UNESP

Celma de Oliveira Ribeiro-POLI-USP

Oswaldo Luiz do Valle Costa-POLI-USP 


\section{Agradecimentos}

A Deus, que dá um sentido a cada momento da minha vida.

A minha mãe, pelo apoio incansável em todos os desafios da minha vida e pelo amor incondicional com que sempre me educou.

À Laura, pelo incentivo contínuo durante muitos momentos difíceis pelos quais passei durante meu doutorado. Que a nossa amizade sempre se fortaleça ao longo de nossos caminhos.

Ao meu orientador, Pedro Paulo, pela paciência com que conduziu sua orientação, principalmente nos últimos meses. Deixo aqui minha gratidão por suas palavras de incentivo e também pelas suas críticas que nunca hesitou em fazer quando necessário e que sem dúvida me conduziram ao verdadeiro aprendizado.

Ao meu pai e meus irmãos pelo apoio sempre presente e pela ajuda em todos os momentos em que precisei.

Ao colega Robson, companheiro de conversas intermináveis, às vezes exaltadas, que só acontecem entre bons amigos.

Ao colega Alessandro, pela boa amizade e pelas seções de piadas que sempre promoveu em nosso apartamento.

Ao Pinho, da secretaria de pós-graduação do IME, por sua constante boa vontade em ajudar, e aos demais funcionários por sempre desenvolverem com muita simpatia e competência suas funções.

Enfim, gostaria de agradecer sinceramente a todos que, de alguma forma, me apoiaram ou contribuíram para que eu conseguisse terminar este trabalho. 


\section{Resumo}

O principal objetivo deste trabalho é construir um modelo de cálculo de risco de crédito que considere efeitos de contágio microeconômico entre os tomadores de um portfólio. Considera-se que tais efeitos ocorrem sob estruturas de relações econômicas que são representadas por realizações de grafos aleatórios com topologias préviamente estabelecidas. Nesse sentido podemos considerar efeitos de contágio microeconômico em um portfólio que modela, por exemplo, uma cadeia ou um centro produtivo (topologia fixas de cadeia ou centro) onde as relações de dependências propriamente ditas são estocásticas.

O principal resultado consistirá em obter a distribuição de perdas do portfólio cuja incerteza reside tanto sobre o estado de default do tomador como nas realizações de contágio microeconômicos que induzem demais defaults. 


\section{Abstract}

The main objective of this work is to construct a model of calculation of credit risk that considers contagion effects microeconomic among the debtor of a portfolio. It is considered that such effects happen under structures of economical relationships that it are represented by accomplishments of random graphs with topologies previously established. In this sense we can to consider effects of infection microeconomics in a portfolio that it models, for instance, a chain or a productive center (topology fixed of chain or center) where the relationships of dependences are stochastics.

The main result will consist of obtaining the distribution of losses of the portfolio whose uncertainty lives on the state of default of the debtor as in the accomplishments of contagion microeconomics that induce other defaults. 


\section{Sumário}

Introdução vi vi v v v

1 Construção do Modelo 1

1.1 Introdução . . . . . . . . . . . . . . . . . . . . . . . . 1

1.2 Pressupostos Básicos e Definições . . . . . . . . . . . . . . 2

1.3 Microestruturas Econômicas . . . . . . . . . . . . 5

1.3.1 Definindo uma Microestrutura Econômica . . . . . . . 5

1.3.2 Atribuindo Incerteza Sobre as Estruturas de

Contágio . . . . . . . . . . . . . . . . . . . 8

1.4 Construção do Modelo de Incerteza . . . . . . . . . . . . . . . 10

1.4.1 O Modelo Básico . . . . . . . . . . . . . . . . . . . 10

1.4.2 Integrando Microestruturas Econômicas . . . . . . . . . . 13

1.4.3 Modelo de Incerteza . . . . . . . . . . . . . . . . . . . . . 19

1.5 Função Geradora da Perda . . . . . . . . . . . . . . . . . . . . 21

2 Topologias de Contágio $\quad 26$

2.1 Introdução . . . . . . . . . . . . . . . . . . . . . . . . 26

2.2 Centros Produtivos Independentes . . . . . . . . . . . . . 28

2.2.1 Modelo de Incerteza . . . . . . . . . . . . . . . . . . 32 
2.2.2 Cálculo da Distribuição de Perdas do Portfólio . . . . . . . 45

2.2.3 Conclusões e Estudos Futuros . . . . . . . . . . . . . 68

Bibliografia 


\section{Introdução}

Gerenciamento de risco de crédito tem sido um tema de discussão crescente na literatura científica. Em termos gerais, o principal objetivo de um modelo de risco de crédito é estimar o risco de inadimplência de tomadores/devedores de um determinado portfólio. Essencialmente busca-se analisar a distribuição de perdas do portfólio extraindo-se informações relevantes para um adequado gerenciamento do mesmo como, por exemplo, o VaR ( Valor em Risco), que é a perda máxima esperada a um determinado nível de confiança, contribuição individual de risco, etc. Existem essencialmente três abordagens utilizadas em modelos de risco de crédito atualmente. A primeira delas são os chamados modelos estruturais ou de firma. Tais modelos são caracterizados por serem essencialmente endógenos, ou seja, nesses casos o risco de inadimplência depende essencialmente da estrutura de capital da empresa devedora ${ }^{1}$, sendo portanto gerado pela dinâmica do valor dos ativos da mesma. A idéia básica é aplicar a teoria de precificação de opções para apreçamento de empréstimos. O trabalho que deu origem a essa linha de pesquisa foi desenvolvido por Merton ( [Merton, 1974]) que apresenta o valor econômico de um empréstimo como uma opção de venda sobre o valor dos ativos da empresa devedora. Neste trabalho, assumindo algumas premissas básicas (dentre as quais, que o mercado é livre de arbitragem, a taxa de juros livre

\footnotetext{
${ }^{1}$ ou seja, capital, dívidas de curto prazo, de longo prazo,etc
} 
de risco é conhecida e constante e que o valor dos ativos da empresa seguem um processo difusivo com dinâmica lognormal ), ele utiliza o modelo de Black-Scholes de precificação de opções ${ }^{2}$ para apreçar um título de dívida(debêndure). Diversas extensões deste trabalho surgiram posteriormente sempre no intuito de reduzir limitações impostas pelo mesmo (Veja por exemplo, [Black,1976],[Vasicek, 1984], [Brys,1997], e [Shimko,1993] que trabalham com taxas de juros estocásticas e [Zhou, 1996] que utiliza um processo de difusão com saltos para a dinâmica do valor da firma). Atualmente o modelo desenvolvido pela KMV Corporation de San Francisco (o Modelo Credit monitor) é baseado no modelo de Merton (veja [Vasicek, 1984]). Uma segunda abordagem de medição de risco de crédito é explorado nos chamados modelos de intensidade (ou de forma reduzida). Esses modelos foram essencialmente introduzidos por [Jarrow,1995] e [Duffie, 1999] e caracterizam-se por focarem a atenção no momento de default ou evento de crédito, ou seja, o momento em que torna-se público ao mercado a incapacidade de uma empresa em honrar sua dívida. Deste modo considera-se o tempo de default como sendo uma variável aleatória positiva e estuda-se sua distribuição de probabilidade. Em geral o tempo de default é modelado por um processo de Poisson cujo parâmetro que representa a intensidade de default é assumido constante, determinístico ou estocástico (este último caso é usualmente chamado de processo de $\operatorname{Cox}^{3}$ ). Um modelo de intensidade usado comercialmente como ferramenta para gerenciamento de risco de crédito é o Credit Risk+ desenvolvido pela Credit Suisse Financial Products ${ }^{4}$.Uma terceira via para modelagem em risco de crédito é representada pelos modelos baseados em classificação por rating. Um modelo

\footnotetext{
${ }^{2}$ ver o clássico artigo em [Black,1973] ou [Hull,1997] para estudos introdutórios deste modelo.

${ }^{3}$ veja [Lando, 1994] para um abordagem deste caso.

${ }^{4}$ veja [Credit Suisse,1997]
} 
de rating classifica as mais diversas instituições(países, empresas, etc.) de acordo com seu risco de inadimplência, por exemplo, AAA, AA, A, AB,..D, onde AAA é o melhor conceito e D é o estado de default. Consequentemente, tais modelos fornecem parâmetros de referência através dos quais os agentes do mercado se baseiam durante suas atividades. Esses parâmetros ou conceitos são conhecidos por ratings e a eles estão associados faixas de probabilidades de um evento de crédito. A especificação dessas faixas é proprietária, ou seja, são especificadas pelas agências classificadoras de risco de crédito. Os modelos de [Jarrow,1997] bem como os modelos CreditMetrics, desenvolvido pela J.P. Morgan ${ }^{5}$ e Credit Portfolio View, desenvolvido pela Mckinsey \& Company ${ }^{6}$ são representativos dessa linha de pesquisa. Para uma pesquisa mais detalhada sobre todos esses modelos veja, por exemplo, [Duffie, 2003], [Schonbucher,2000], [Bielecki,2002] ou [Christian,2000].

Em termos gerais, podemos classificar modelos de risco de crédito conforme a estrutura de correlações assumida entre os eventos de default. A hipótese mais forte a ser considerada é a suposição de independência incondicional entre os eventos de default do portfólio . Em outras palavras, supõe-se que os eventos de default não estão correlacionados por quaisquer fatores específicos da economia, o que obviamente é pouco realístico. Modelos mais sofisticados trabalham com a hipótese de independência condicional. Nesse caso considera-se a existência de variáveis latentes representando fatores sistêmicos de risco que induzem correlações ( por exemplo, estados da economia, região geográfica, setor industrial, etc). A independência condicional se caracteriza pelo fato de que, uma vez revelados os estados dos fatores sistêmicos de risco, os eventos de default tornam-se

\footnotetext{
${ }^{5}$ Veja [Morgan,1996].

${ }^{6}$ Baseado no trabalhos de Wilson em [Wilson,1997a] e [Wilson,1997b].
} 
independentes, ou seja, a probabilidade de ocorrência de dois eventos condicionais é o produto de suas probabilidades. A maioria dos modelos conhecidos, dentre os quais os citados acima, trabalham nesse contexto. Um tratamento mais complexo sobre correlações ocorre quando sugere-se a hipótese de dependência condicional sobre os eventos de default. Nesse contexto, busca-se modelar, além de fatores sistêmicos de risco, também dependências explícitas entre os eventos de default, como por exemplo, contágio micreoconômico entre firmas, garantias, colaterais, etc. Como dissemos anteriormente, em geral os modelos atuais trabalham com a hipótese de independência condicional, de forma que as correlações estimadas refletem apenas fatores de natureza macroeconômica afetando um mesmo grupo de tomadores . De fato, em portfólios pouco diversificados com relação a setores econômicos ou geográficos os eventos de default são correlacionados. No entanto, certamente efeitos semelhantes podem ocorrer em casos onde exista dependências de natureza puramente microeconômica entre os tomadores do portfólio. Pode-se perceber claramente isso, por exemplo, em cenários de cadeias produtivas, onde a falência de uma grande empresa pode afetar diretamente seus fornecedores de matéria prima. Em nosso trabalho propomos um modelo que considera exatamente esses tipos explícitos de dependências.

Modelagem de risco de crédito considerando dependências microeconômicas é um assunto recente na literatura acadêmica mas vem sendo estudado de forma crescente nos últimos anos . Entre os trabalhos mais recentes podemos citar [Jarrow,2001] onde é generalizado um modelo de intensidade apresentado em [Lando, 1994], e considera intensidade de default dependente sobre o default de uma contra-parte. Inicialmente é assumido que a intensidade de default de uma firma é dependente sobre o estado de default de outras firmas num contexto geral, 
mas devido a um problema que os autores chamam de "looping default", dificultando a obtenção distribuição conjunta do tempo de default, uma restrição é imposta sobre o modelo proposto. É assumido então uma estrutura de dependências assimétricas entre firmas onde um conjunto de firmas chamadas de primárias influencia os defaults de um segundo conjunto de firmas secundárias ( e a recíproca não é válida). Recentemente [Egloff, 2004] usou teoria de grafos para considerar relações econômicas particulares em um modelo setorial do tipo vasicek e usa simulação de Monte Carlo para analisar os efeitos de diferentes estruturas de interdependências microeconômicas sobre a distribuição de perdas de um portfólio. Sua abordagem não é muito apropriada para o tratamento de grandes portfólios devido à complexidade computacional para simulação de grafos. Gieseck e Weber em [Giesecke,2004] estudaram contágio econômico em risco de crédito utilizando como base teoria de interações de sistemas de partículas. Neste trabalho os autores incorporam os efeitos de contágio em um portfólio homogêneo de tamanho n (isto é, n tomadores com igual exposição ao risco e igual probablidade de default incondicional), obtendo uma aproximação assintótica para a distribuição de perdas do portfólio para n grande. Em [Giesecke,2003] o estudo é estendido considerando também a existência de correlações em períodos cíclicos induzidas por fatores macroeconômcos. Estudos no contexto de portfólios homogêneos também são abordados em outros trabalhos como ([Frey,2002]) que utilizam técnicas de Processo de Markov e teoria de Campo Médio e [Neu,2004] que generaliza um modelo estrutural para capturar o impacto de defaults de contra-partes sobre a distribuição de perdas de um portólio de crédito. Este último trabalho é posteriormente estendido em [Hatchett,2006] que lida, neste caso, com porfólios heterogêneos. Os autores trabalham em um caso limite particular onde o número de 
interações microeconômicas de um particular tomador é assintóticamente nulo ( um regime de "extrema diluição" do risco microeconômico).

Em termos gerais podemos dizer que as contribuições dos autores citados promovem um avanço significativo no estudo do contágio microeconômicos sob ponto de vista teórico, mas pecam sob o ponto de vista prático em não fornecer uma caracterização explícita da distribuição de perdas de um portfólio finito heterogêneo com exposições e probabilidades de default distintas e que leva em conta efeitos de contágio microeconômico reflitindo de forma simples relações econômicas que passam a existir entre os tomadores de um portfólio. Uma tal caracterização permite elaborar uma ferramenta prática de gestão de risco de crédito que possibilita ao gestor conduzir testes de stress em seu modelo de risco que reflitam cenários de crises onde os efeitos das relações microeconômicas dos tomadores produzem perdas extremas.

A principal contribuição deste trabalho é de exatamente generalizar o modelos de cálculo de risco de crédito em um paradigma atuarial de default para incluir efeitos de contágio microeconômicos que se dão sob uma estrutura cujo cerne de relações econômicas, traduzidas aqui em realizações de grafos aleatórios nos tomadores do porfólio, tem uma topologia préviamente determinada e fixa. Assim poderemos considerar efeitos de contágio microeconômico em um portfólio que modela, por exemplo, uma cadeia ou um centro produtivo (topologia fixas de cadeia ou centro) onde as relações de dependências propriamente ditas são estocásticas.

O principal resultado consistirá em obter a distribuição de perdas de um portfólio que incorpora tanto a incerteza no estado de default do tomador como as realizações dos canais de contágio microeconômicos que induzem demais de- 
faults.

Iremos considerar um portfólio onde os tomadores estão sujeitos a somente dois possíveis eventos; default e não default, onde o evento de default significa a ocorrência de sua inadimplência ao final de um período de um ano. Consideraremos também conhecidas as probabilidades individuais incondicionais de default de cada um dos tomadores do porfólio estimadas por um gestor de portfólio. Nesse contexto a incerteza que reside na perda do portfólio consistirá nas diversas configurações de default admissíveis sobre os tomadores do portfólio e os possíveis cenários microeconômicos que possam existir entre eles.

Formalizaremos nosso modelo de incerteza primeiramente definindo sobre um portfólio de crédito $\Lambda=\{1, . ., n\}$ de $n$ tomadores e com exposições $\left\{e_{1},,, e_{n}\right\}$, uma microestrutura econômica representada por um grafo simples $g(\Lambda, \vec{J})$ cujos vértices correspondem aos tomadores de $\Lambda$ e as arestas, representadas por $\vec{J}=\left(J_{i j}\right)_{i, j}, J_{i j} \in \mathbb{R}$, indicam a existência de interações microeconômicas entre ambos.

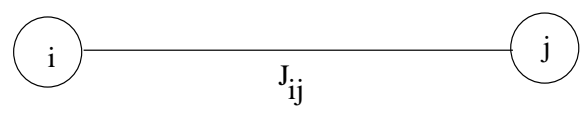

Em todo o trabalho a topologia dos grafos será conhecida e fixada a priori. Também nos restringiremos a estudar microestruturas $g(\Lambda, \vec{J})$ com contágio uniforme, ou seja, assumiremos que

$$
\vec{J}=\left(J_{i j}\right)_{i, j=1}^{n}, \quad J_{i j}=J \delta_{i j}, \quad i, j \in \Lambda
$$

onde

$$
\delta_{i j}=\left\{\begin{array}{llr}
1 & \text { se } & \text { existe interação entre i e j } \\
0 & \text { se } & \text { não existe interação entre i e j }
\end{array}\right.
$$

para algum $J \in \mathbb{R}^{+}$.

Atribuiremos incerteza sobre as microestruturas econômicas fazendo uso do 
conceito de grafos aleatórios binomiais ou de Erdös e Rényi (ver [Erdos,1959]). Nesse contexto sortearemos microestruturas econômicas considerando ( $\left.\begin{array}{c}n \\ 2\end{array}\right)$ sorteios independentes sobre os pares $(i, j)$ de tomadores do portfólio, onde a probabilidade de existência de interação econômica entre dois tomadores $\left(J_{i j}=J\right)$ é sempre igual a uma constante $c \in[0,1]$. Em outras palavras, sobre uma classe $G(\Lambda, c, J)$ de microestruturas a serem sorteadas (que chamaremos de microestrutura aleatória) definiremos a medida de probabilidade

$$
\begin{aligned}
\mathbb{Q}: G(\Lambda, c, J) & \longrightarrow[0,1] \\
g & \longmapsto \mathbb{Q}(g)=c^{M(g)}(1-c)^{\left(\begin{array}{c}
n \\
2
\end{array}\right)-M(g)}
\end{aligned}
$$

onde $M(g)$ significa o número de interações microeconômicas existentes em $g(\Lambda, \vec{J})$

Sob essas considerações nosso modelo de incerteza será caracterizado por um espaço de probabilidade $(\mathcal{N}, \mathcal{P}(\mathcal{N}), \mathbb{P})$ consistindo de um espaço de estados

$$
\mathcal{N}=\Omega \times G(\Lambda, c, J)
$$

que representa os estados $\eta=(\omega, g) \in \Omega \times G(\Lambda, c, J)$, com

$$
\Omega=\left\{\omega=\left(\omega_{i}\right)_{i=1}^{n}, \omega_{i} \in\{-1,+1\}\right\}
$$

modelando os estados de default, isto é, para cada $i=1, . ., n$ temos que:

$$
\omega_{i}=\left\{\begin{array}{ccc}
+1 & \text { se } & i \text { default } \\
-1 & \text { se } & i \text { não default }
\end{array}\right.
$$

Motivados por teria baseada em mecânica estatística (específicamente o modelo de Ising que explicaremos no capítulo 1), definiremos uma medida de probabilidade sobre o espaço de configurações de default do portfólio, $\mathbb{P}: \mathcal{P}(\mathcal{N}) \mapsto$ $[0,1]$, como:

$$
\mathbb{P}(\eta)=\mathbb{P}(\omega, g)=\mathbb{P}_{g}(\omega) Q(g)
$$


para todo $\eta=(\omega, g) \in \mathcal{N}$, onde $\mathbb{P}_{g}: \mathcal{P}(\Omega) \mapsto[0,1]$ é dado por

$$
\mathbb{P}_{g}(\omega)=\frac{1}{Z(g)} e^{\alpha \cdot \omega-\frac{1}{2} \sum_{(i j) \in g} J_{i j} \omega_{i} \omega_{j}}
$$

com

$$
Z(g)=\sum_{\omega \in \Omega} e^{\alpha \cdot \omega-\frac{1}{2} \sum_{(i j) \in g} J_{i j} \omega_{i} \omega_{j}}, \quad \alpha \cdot \omega=\sum_{i=1}^{n} \alpha_{i} \omega_{i} .
$$

sendo $\alpha=\left(\alpha_{i}\right)_{i=1}^{n}$ um parâmetro que depende das probabilidades individuais incondicionais de default de cada um dos tomadores do portfólio ( que lembrando temos a priori).

Na definição acima a medida $\mathbb{P}_{g}$ atribui probabilidade as configurações de default debaixo de uma realização g do grafo que representa o contágio.

Os resultados neste trabalho serão baseados no cálculo de aproximação de ponto de sela para a distribuição de perdas L, onde $L: \mathcal{N} \rightarrow \mathbb{R}^{+}$em $(\mathcal{N}, \mathcal{P}(\mathcal{N}), \mathbb{P})$ é definida como

$$
L(\eta)=\sum_{i=1}^{n} e_{i} \mathbb{L}_{i}(\eta)
$$

com

$$
\mathbb{L}_{i}(\eta)=\left\{\begin{array}{lll}
1 & \text { se } & \omega_{i}=+1 \\
0 & \text { se } & \omega_{i}=-1
\end{array}\right.
$$

para todo $\eta=(\omega, g) \in \mathcal{N}$.

Isto implica em calcular a função cumulante da perda L, isto é, o logaritmo de sua função geradora $G(z)$ :

$$
\begin{aligned}
G(z) & =E\left[e^{z L}\right] \\
& =\sum_{\eta \in \mathcal{N}} e^{z L(\eta)} \mathbb{P}(\eta)
\end{aligned}
$$

O principal problema que encontramos para utilizar G(z) é que, como podemos observar, sua expressão é definida como uma soma de complexidade exponencial o que é inviável para um tratamento numérico (observe que sobre $\Omega$ existem 
$2^{n}$ configurações estados de dafault possíveis e sobre $G(\Lambda, c, J)$ temos um total de $2^{\frac{n(n-1)}{2}}$ configurações microestruturais). Mostraremos, no entanto, que para um estudo de sensibilidade ou stress, podemos resolver satisfatoriamente esse problema evitando os cálculos exponenciais.

Para isto, particularmente trabalharemos com topologias fixas do tipo centro produtivo onde as interações microeconômicas se caracterizam por um tomador central interagindo com um grupo de tomadores "satélites" como mostra a figura abaixo:

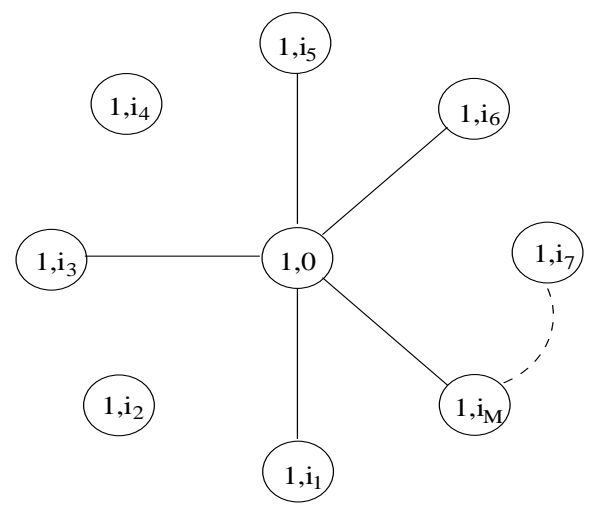

Consideraremos as interações sendo pequenas, o que implicará em uma drástica simplificação sobre a expressão de G(z) em (12), aparecendo expressões de complexidade polinomial.

Nesse contexto nosso modelo de incerteza será particularizado considerando o espaço produto $\mathcal{N}=\Omega \times G(\Lambda, c, J)$ onde consideramos a microestrutura aleatória $G(\Lambda, c, J)$ como uma classe cujas únicas microstruturas a serem sorteadas são as do tipo centro produtivo.

Assim, quando calculamos (12) obtemos uma série de siplificações algébricas 
que conduzem a uma expressão do tipo

$$
G(z)=\sum_{M=0}^{n} \sum_{i_{1}<i_{2}<. .<i_{M}} G\left(z,\left(i_{l}\right)_{M},\left(j_{k}\right)_{N}, J\right) c^{M}(1-c)^{n-M}
$$

onde $G\left(z,\left(i_{l}\right)_{M},\left(j_{k}\right)_{N}, J\right)$ é uma função analítica em z, explícitamente determinada e a soma em (13) corresponde a todos as listas de tomadores $\left\{i_{1}, . ., i_{M}\right\}$ interagindo com um tomador central do portfólio considerado.

Observe que mesmo nos restringindo a microestruturas de centro produtivo a expressão da função geradora ainda se apresenta como uma soma de ordem exponencial (embora menos complexa como no caso geral). Podemos afirmar que para $J=0$ a expressão de $G\left(z,\left(i_{l}\right)_{M},\left(j_{k}\right)_{N}, J\right)$ se torna independente dos índices da soma em (13) resultando em uma função $\tilde{G}(z)$ que só depende de z. Nesse caso a soma binomial em (13) é eliminada pois:

$$
\begin{aligned}
G(z) & =\sum_{M=0}^{n} \sum_{\substack{i_{1}<i_{2}<\ldots<i_{M} \\
j_{1}<j_{2}<\ldots<j_{n-M}}} G\left(z,\left(i_{l}\right)_{M},\left(j_{k}\right)_{N}, J\right) c^{M}(1-c)^{n-M}= \\
& =\tilde{G}(z) \sum_{\substack{M=0}} \sum_{\substack{i_{1}<i_{2}<\ldots<i_{M} \\
i_{1}<. .<j_{n-M}}} c^{M}(1-c)^{n-M} \\
& =\tilde{G}(z)(c+(1-c))^{n} \\
& =\tilde{G}(z) .
\end{aligned}
$$

É razoavel esperar que simplifições desse tipo possam também acorrer com derivadas parciais (com respeito a $J$ ) de $G(z)$ em $J=0$. Nesse sentido, um estudo baseado em expansões assintóticas em torno de $J=0$ parece bastante sugestivo. De fato, em nosso trabalho utilizaremos essa abordagem para refinar o método de ponto de sela para aproximação da cauda da distribuição de perdas de um portfóio de crédito cujos tomadores interagem entre si, em média, conforme 
uma estrutura de centro produtivo. Nossa aproximação resultará em uma expressão analítica para a probabilidade acumulativa $\mathbb{P}(L>t)$ cuja complexidade se reduzirá a expressões de ordem de magnitude $O(n), O\left(n^{2}\right)$ e $O\left(n^{3}\right)$, tornando o modelo totalmente viável para estimar o $V a R$ " estressado" da carteira de crédito considerada. Formalmente apresentamos o seguinte resultado:

Teorema Considere um portfólio de crédito $\Lambda=\{0,1, . ., n\}$ com exposições $\left\{e_{1},,, e_{n}\right\}$ e probabilidades individuais de default $\left\{p_{1},,, p_{n}\right\}$ com uma microestrutura de centro produtivo $G(\Lambda, c, J)$ e $L: \mathcal{N} \mapsto \mathbb{R}^{+}$a perda associada ao portfólio $\Lambda$ definida no espaço de probabilidade $(\mathcal{N}, \mathcal{P}(\mathcal{N}), \mathbb{P})$, onde $\mathbb{P}: \mathcal{P}(\mathcal{N}) \mapsto[0,1]$ é definida em (7). Então, dado $t \in \mathbb{R}^{+}$, existe $\xi_{t} \in \mathbb{R}$ tal que, para a distribuição de perdas do portfólio $\Lambda$ em torno de $J=0$, a probabilidade cumulativa $\mathbb{P}(L>t)$ resulta em uma função dada pela seguinte expressão:

$$
\mathbb{P}(L>t)=\exp \left(\Theta_{1}\left(\xi_{t}, J\right)-t \xi_{t}+\frac{1}{2} \xi_{t}^{2} \Theta_{2}\left(\xi_{t}, J\right)\right) N\left(-\sqrt{\xi_{t}^{2} \Theta_{2}\left(\xi_{t}, J\right)}\right)
$$

com $N$ sendo a função de distribuição normal acumulativa e

$$
\begin{aligned}
& \Theta_{1}\left(\xi_{t}, J\right)=X^{(0)}\left(\xi_{t}\right)+X^{(1)}\left(\xi_{t}\right) J+\frac{1}{2} X^{(2)}\left(\xi_{t}\right) J^{2} . \\
& \Theta_{2}\left(\xi_{t}, J\right)=Z^{(0)}\left(\xi_{t}\right)+Z^{(1)}\left(\xi_{t}\right) J+\frac{1}{2} Z^{(2)}\left(\xi_{t}\right) J^{2} .
\end{aligned}
$$

e as funções $\Theta_{1}\left(\xi_{t}, J\right)$ e $\left.\Theta_{2}\left(\xi_{t}, J\right)\right)$ são polinômios quadráticos (em J), cujos coeficientes são expressos com somas de ordem de magnitude $O(n), O\left(n^{2}\right)$ ou $O\left(n^{3}\right)$.

Para alcançar o resultado enunciado desenvolveremos o trabalho seguindo o roteiro a seguir. No capitulo 1 enunciaremos na seção 1.2 alguns pressupostos básicos que servirão de alicerce para definirmos o modelo proposto. Antes de propriamente definirmos nosso modelo de incerteza formalizaremos na seção 1.3 
uma estrutura para representar adequadamente dependências microeconômicas entre devedores de uma carteira de crédito bem como uma adequada abordagem para representar incerteza sobre tais relações. Uma vez formalizado o contexto econômico a ser trabalhado, na seção 1.4 construiremos nosso modelo de incerteza, iniciando na seção 1.4.1 com um modelo básico supondo eventos de default independentes e posteriormente nas seções 1.4.2 e 1.4.3 incorporaremos dependências entre os eventos de default, primeiro sugerindo estruturas microeconômicas fixas e depois atribuindo incerteza sobre as estruturas de contágio. Na seção 1.5 analisaremos a função geradora da perda, uma ferramenta importante para estudarmos a distribuição de perdas de um portfólio de crédito no segundo capítulo.

Após termos estruturado o modelo em um contexto generalizado no segundo capítulo, no capítulo 3 será feito uma contextualização do modelo para um cenário particular caracterizado por uma topologia de centro produtivo. Finalmente na seção 3.2.2 mostraremos sua viabilidade para estimar o VaR de um portólio de crédito estressado particularmente por um cenário de centro produtivo. 


\section{Capítulo 1}

\section{Construção do Modelo}

\subsection{Introdução}

Nesse capítulo temos como principal meta construir o modelo de incerteza que utilizaremos para análise de risco de crédito. Precisamente, nos concentraremos em definir uma medida de probabilidade que captura efeitos de contágio econômico entre devedores da carteira. Para fazer isso, na seção 1.2, alguns pressupostos básicos serão primeiramente abordados, como a definição de inputs do modelo e certas características sujacentes a sua dinâmica. Na seção 1.3 formalizaremos uma estrutura para representar dependências microeconômicas explícitas entre tomadores de um portfólio e posteriormente atribuiremos incerteza sobre essas estruturas com vistas a futuramente definirmos nosso modelo. O próximo passo, na seção 2.4, será propriamente definir nosso modelo de incerteza iniciando com um modelo básico e, posteriormente, considerando estruturas de dependências microeconômicas entre os elementos do portfólio (seções 1.4.2 e 1.4.3 ). Na última seção terminaremos o capítulo por conceituando a função geradora de momentos relativo ao portfólio estudado, um elemento fundamental que utilizaremos no segundo capítulo para analizarmos a distribuição de perdas do portfólio considerando uma estrutura particular. 


\subsection{Pressupostos Básicos e Definições}

Nessa seção apresentaremos um primeiro bloco básico de questões que julgamos ser relevante a abordagem. Enunciaremos algumas hipóteses que servirão de ponto de partida para a construção de nosso modelo probabilístico abordando essencialmente três questões. Primeiramente enfatizaremos uma suposição determinística para as exposições em default com um breve comentário sobre o assunto. Em seguida descreveremos como o processo de crédito estará subentendido no modelo, carcterizando-o como um modelo de inadimplência. Por último assumiremos conhecidas as probabilidades individuais de default de cada exposição da carteira de crédito, um input básico para o modelo.

Hipótese 1.1 Exposições ao default são quantidades determinísticas (ou seja, não aleatórias)

A hipótese 1.1 não é uma suposição totalmente natural pois uma fração de exposições ao default pode ser formada por recursos que foram disponibilizados pelo banco mas não necessariamente sacados pelo cliente (linhas de crédito, por exemplo). Isto atribui um caráter aleatório às exposições já que há incerteza sobre qual parcela será sacada antes de um possível default de um cliente. Existe uma série de detalhes subjacentes a essa incerteza. Por exemplo, em muitos casos os bancos incluem algumas condições (covenants) sobre linhas de crédito fornecidas. Tais condições podem, por exemplo, forçar o cliente a fornecer um colateral em situações de stress financeiro ou renegociar os termos do contrato. O banco também pode impor a condição de cancelar a linha de crédito do cliente caso haja alguma indicação de default. No entanto, o sucesso dessas medidas preventivas estão condicionadas à capacidade do banco fazer uso das mesmas no 
momento certo, já que situações de default nem sempre são facilmente detectadas, especialmente no caso de bons clientes. Uma abordagem estocástica para as exposições teria que levar em consideração questões desse tipo. Neste trabalho evitaremos tais particularidades, uma vez que nosso foco é estudar o efeito de dependências microeconômicas em portfólios de crédito e a hipótese 2.1 constitui uma simplificação conveniente.

Hipótese 1.2 Todo devedor de crédito está sujeito a somente dois possíveis eventos; default e não default, onde o evento de default significa a ocorrência de sua inadimplência ao final de um período de um ano.

A hipótese 1.2 significa que o modelo proposto neste trabalho se caracteriza como um modelo de inadimplência, ou seja, apenas o momento de default nos interessa. Portanto vamos ignorar eventuais mudanças na qualidade do crédito de um devedor ao longo do período do empréstimo e também não faremos quaisquer suposições sobre possíveis causas do default, assumindo o evento de default como um processo exógeno (ou seja, sem relação direta com a estrutura de capital do devedor). A escolha de um período de tempo constante de um ano para maturidade dos empréstimos também é relevante pois permite que todas as exposições sejam consideradas em uma mesma data futura, além de existirem também outras razões aconômicas para isto (veja [Credit Suisse,1997], pag. 11).

Hipótese 1.3 (probabilidade individual incondicional de default) Cada tomador de empréstimo está associado a uma função de probabilidade $\mathbb{P}_{d}: \mathcal{F} \rightarrow[0,1]$ definida sobre um conjunto de eventos mensuráveis $\mathcal{F}$ que contém o evento de default ${ }^{1}$. Além disso, definimos $p=\mathbb{P}_{d}($ default $)$, para algum $p \in(0,1)$, como a

\footnotetext{
${ }^{1}$ o termo " evento de default"para nós sempre significará a inadimplência do tomador ao final
} 
probabilidade incondicional de default do tomador correspondente.

Como pressuposto básico para nosso modelo estamos assumindo conhecidas as probabilidades individuais incondicionais de default de cada devedor. A incondicionalidade se deve ao fato de que as mesmas não estão condicionadas a nenhum fator específico da economia, seja de natureza microeconômica ou macroeconômica. Por uma questão de simplicidade, no que diz respeito a manipulações que teremos de fazer futuramente, as probabilidades individuais de default para nós serão sempre consideradas estritamente positivas e menores que um, o que é bastante razoável pois considerar um devedor como livre de risco $(\mathrm{p}=0)$ mesmo tendo grande credibilidade junto a instituição cedente do empréstimo não é uma prática muito recomendável e muito menos recomendável seria também considerar um portólio cujo devedor tenha probabilidade de default certo $(\mathrm{p}=1)$.

Probabilidades individuais de default são divulgadas por agências externas especializadas ou calculadas por instituições bancárias que utilizam suas próprias metodologias internas. Uma forma de cálculo muito utilizada, por exemplo, é a utilização de sistemas de classificação por ratings. Os ratings basicamente descrevem graus de credibilidade para tomadores de crédito (por exemplo, AAA, AA, BBB,.etc). Então extrai-se taxas históricas de default associadas a cada faixa de classificação e as atribui aos devedores correspondentes. Existe toda uma teoria sobre calibração de probabilidades de default que não abordaremos aqui.

do contrato de empréstimo (1 ano como convencionamos) 


\subsection{Microestruturas Econômicas}

Antes de definirmos nosso modelo de incerteza, nesta seção costruiremos uma estrutura que represente adequadamente cenários microeconômicos evidenciando dependências explícitas entre os tomadores de um portólio de crédito. Primeiro conceituaremos cenários fixos de contágio microeconômico e posteriormente formalizaremos uma abordagem aleatória sobre tais estruturas, o que nos permitirá, futuramente, definirmos um modelo probabilístico que possa medir realizações microetruturais.

\subsubsection{Definindo uma Microestrutura Econômica}

Nessa seção definiremos formalmente o que interpretaremos como uma estrutura microeconômica a ser incorporada em nosso modelo de incerteza. Esse conceito será estabelecido levando em consideração relações puramente microeconômicas entre devedores de uma carteira de crédito. Isto significa que não consideraremos explícitamente fatores sistemáticos ou macroeconômicos influenciando as exposições, como por exemplo, região geográfica, indicadores econômicos, situação econômica de um país, etc. A seguir formalizaremos esse contexto que pretendemos trabalhar.

Definição 1.1 (interação microeconômica) dizemos que existe interação microeconômica entre dois devedores de um portfólio de crédito se a deterioração ou melhora financeira de um implica diretamente deterioração ou melhora financeira do outro.

Observe que, ao contrário de correlações originadas implicitamente por fatores comuns a dois tomadores de um portfólio, uma interação microeconômica 
se caracteriza como uma correlação explícita já que surge de efeitos diretos que podem existir entre duas contrapartes. Uma interaçao econômica entre dois devedores pode ser tanto cooperativa quanto competitiva. A relação é cooperativa quando a deterioração/melhora financeira de um implica deterioração/melhora do outro. Essa última situação caracteriza o que chamamos de contágio de crédito em que o aumento da probabilidade de default de um aumenta a probabilidade de default do outro devido à dependência econômica direta existente entre eles. Já no caso de uma relação competitiva a piora/melhora financeira de um implica melhora/piora financeira do outro. Como exemplo podemos considerar, uma grande fabricante de $\mathrm{PC}^{\prime}$ s. A falência da fábrica evidentemente afetaria positivamente suas concorrentes nesse setor industrial. Por outro lado o efeito seria negativo sobre fornecedores de acessórios ou provedores de serviços para os $\mathrm{PC}$ 's vendidos pela empresa. Como veremos adiante, nos restringiremos mais aos casos de cooperação microeconômica entre os devedores de um portfólio como contexto para construção do modelo.

A seguir formalizaremos essas idéias em uma estrutura de contágio microeconômico uniforme. Isto quer dizer que as dependências microeconômicas, quando existirem, serão sempre consideradas com uma mesma intensidade previamente definida. Essa suposição de maneira alguma enfraquece o modelo, haja vista que nossa proposta é estudar o efeito de pequenas interações dentro de um portfólio, o que pode ser plenamente realizado nesse contexto.

Definição 1.2 Dado um portfólio de crédito $\Lambda=\{1, n\}$, indicaremos a existência de interação microeconômica entre dois tomadores de $\Lambda$ pelos elementos da 
matriz:

$$
\vec{J}=\left(J_{i j}\right)_{i, j=1}^{n},
$$

cujos elementos são definidos como

$$
J_{i j}=J \delta_{i j}, \quad J \in \mathbb{R}^{+}
$$

para todo $i, j \in \Lambda$, onde:

$$
\delta_{i j}=\left\{\begin{array}{llr}
1 & \text { se } & \text { existe interação entre } i \text { e } j \\
0 & \text { se } & \text { não existe interação entre } i \text { e } j
\end{array}\right.
$$

Os parâmetros $J_{i j}$ representam a intensidade de interação microeconômica existente entre $i$ e $j$ e satisfazem:

1) $J_{i j} \geq 0$ para todo $i, j \in \Lambda$

2) $J_{i j}=J_{j i}$ para todo $i, j \in \Lambda$.

3) $J_{i i}=0$ para todo $i \in \Lambda$.

4) $S e J_{i j}=0$ e $i \neq j$, então não existe interação microeconômica entre $i$ e $j$.

Conforme definimos acima, em nosso trabalho nos restringiremos a sugerir correlações microeconômicas sempre positivas entre os tomadores de uma carteira de crédito. Nesse caso, para cada $i, j \in \Lambda, J_{i j}$ pode ser considerado, por exemplo, como volume de negócios entre os dois tomadores, o que caracterizaria uma relação de cooperação entre firmas. Na verdade essa restrição nos insere mais num contexto de contágio microeconômico entre tomadores de um portfólio, onde, como dissemos anteriormente, o default de um tomador influencia positivamente o default de outros. No entanto não entraremos diretamente no mérito desses detalhes haja vista que não pretedemos calibrar esses parâmetros e sim apenas considerá-los como ferramenta para testes de sensibilidade de uma carteira de crédito a cenários microeconômicos. Por essa mesma razão desconsideraremos 
outros detalhes no que concerne a relações micreoconômicas como a possibilidade de existir alguma ordem nas relações econômicas entre duas contrapartes (item 2)). Naturalmente também estamos supondo que uma firma não se relaciona consigo mesma (item 3).

Uma vez definidos os parâmetros de contágio o próximo passo será criar uma representação para uma estrutura particular de dependências sobre o portfólio $\Lambda$. Esse procedimento será feito utilizando grafos simples. ${ }^{2}$

Definição 1.3 Definimos uma microestrutura para o portfólio de crédito $\Lambda=$ $\{1, . ., n\}$ como um grafo simples $g(\Lambda, \vec{J})$ cujos vértices correspondem às contrapartes de $\Lambda$ e as arestas, representadas pelos elementos de $\vec{J}$, definido em (1.1), indicam a existência de interações microeconômicas entre ambas.



Uma vez que criamos uma estrutura representativa para cenários microeconômicos fixos, pretendemos, na próxima seção, definir estocásticamente uma microestrutura econômica.

\subsubsection{Atribuindo Incerteza Sobre as Estruturas de Contágio}

Na sessão anterior conceituamos uma estrutura de contágio microeconômico sobre o portfólio $\Lambda$, cuja representação foi feita por um grafo fixo. Naturalmente, no contexto em que foi definido, existe incerteza sobre a microstrutura econômica definida sobre $\Lambda$, já que existem várias possibilidades de dependências

\footnotetext{
${ }^{2}$ Um grafo simples é um par de conjuntos $\mathrm{G}=(\mathrm{V}, \mathrm{E})$ em que os elementos de $\mathrm{V}$ são chamados de vértices e os elementos de E são chamados de arestas. A propriedade básica de uma aresta em um grafo é que ela conecta dois vértices. Formalmente identifica-se uma aresta pelos dois vértices os quais ela conecta. Para um um estudo mais detalhado veja, por exemplo, [Bollobás,1998] e [Diestel,2000].
} 
entre os elementos de $\Lambda$. No caminho para construir uma dinâmica microestrutural em nosso trabalho faz-se necessário escolhermos adequadamente um modelo para medir (no sentido probabilístico) um espaço de configurações microestruturais. Formalizaremos isso usando a idéia de grafos aleatórios binomiais ou de Erdos Reny (ver [Erdos,1959]) devidamente adaptada para o contexto em que estamos trabalhando, conforme definiremos a seguir.

Definição 1.4 Dado um portfólio de crédito $\Lambda$, de $n$ tomadores, definimos uma microestrutura aleatória $G(\Lambda, c, J), c \in[0,1], J \in \mathbb{R}^{+}$como um espaço de probabilidade $(\mathcal{M}, \mathcal{F}, \mathbb{Q})$ onde $\mathcal{M}$ é o espaço de eventos formado por todas as possíveis microestruturas $g(\Lambda, \vec{J})$, com contágio uniforme $J$ sobre $\Lambda, \mathcal{F}$ é a $\sigma$-algebra formada pelos subconjuntos de $\mathcal{M}$ e $\mathbb{Q}$ é a medida de probabilidade definida por:

$$
\begin{aligned}
\mathbb{Q}: \mathcal{M} & \longrightarrow[0,1] \\
S & \longmapsto \mathbb{Q}(S)=\sum_{g \in S} c^{M(g)}(1-c)^{\left(\begin{array}{c}
n \\
2
\end{array}\right)-M(g)}
\end{aligned}
$$

Observe na definição 1.4 que uma microestrutura aleatória pode ser interpretada como uma classe de microestruturas que são geradas por um um processo de $\left(\begin{array}{c}n \\ 2\end{array}\right)$ sorteios independentes sobre as pares de tomadores do portfólio, onde a probabilidade de existir interação entre quaisquer um deles é sempre igual a $c \in[0,1]$. Naturalmente como os sorteios são independentes temos que a probabilidade de existir uma determinada microestrutura é o produto das probabilidades relativas a cada um de seus pares. Por exemplo, temos que a probabilidade de ocorrência de uma microestrutura $g(\Lambda, \vec{J})$ com 3 interações existentes é dada por $c^{3}(1-c)^{\left(\begin{array}{c}n \\ 2\end{array}\right)-3}$.

Na próxima seção iniciaremos a costrução do modelo proposto. Como veremos adiante, a estrutura aleatória estabelecida nesta seção será usada para carac- 
terizar incerteza microeconômica sobre nosso modelo probabilístico.

\subsection{Construção do Modelo de Incerteza}

\subsubsection{O Modelo Básico}

Sob as hipóteses feitas na seção 2.2 consideremos um portfólio $\Lambda=\{1,,, . n\}$ de $\mathrm{n}$ tomadores sujeitos a risco de crédito e com exposições $\left\{e_{i}\right\}_{i=1}^{n}$. Pela hipótese 2.3 também temos associado a cada exposição de $\Lambda$ probabilidades individuais incondicionais de default $\left\{p_{i}\right\}_{i=1}^{n}$.

Como, pela hipótese 2.3, estamos admitindo somente dois eventos possíveis para cada tomador de $\Lambda$ (default ou não default), a incerteza sobre o portfólio ocorre sobre as $2^{n}$ possíveis realizações conjuntas de eventos conforme formalização a seguir.

Definição 1.5 Dado um portfólio de crédito $\Lambda=\{1,,, n\}$ de $n$ tomadores, definimos o conjunto de estados de default sobre $\Lambda$ como o conjunto:

$$
\Omega=\left\{\omega=\left(\omega_{i}\right)_{i=1}^{n}, \quad \omega_{i} \in\{-1,+1\}\right\}
$$

onde, $\omega_{i}, i=1, . ., n$ são variáveis binárias dadas por

$$
\omega_{i}=\left\{\begin{array}{ccc}
+1 & \text { se } & i \text { default } \\
-1 & \text { se } & \text { i não default }
\end{array}\right.
$$

Como suposição inicial consideremos a seguinte hipótese:

Hipótese 1.4 Os eventos de default associados aos devedores da carteira $\Lambda$ são independentes.

Sob a hipótese 2.4, naturalmente podemos definir a função de probabilidade para medir as diversas realizações de default associadas a $\Lambda$ como produto das probabilidades incondicionais de default. 
Definição 1.6 Dado um portfólio de crédito $\Lambda$ de $n$ tomadores com probabilidades individuais incondicionais de default dadas por $\left\{p_{i}\right\}_{i=1}^{n}$, definimos sobre o conjunto de estados, $\Omega$, definido em $(1.5)$, o espaço de probabilidade $\left(\Omega, \mathcal{P}(\Omega), \mathbb{P}_{I}\right)$ com função de probabilidade $\mathbb{P}_{I}: \mathcal{P}(\Omega) \mapsto[0,1]$, dada por

$$
\mathbb{P}_{I}(A)=\sum_{\omega \in A} \prod_{\substack{\omega_{i}=1 \\ \omega_{j}=-1}} p_{i}\left(1-p_{j}\right)
$$

para todo $A \in \mathcal{P}(\Omega)$, onde $\mathcal{P}(\Omega)$ representa o conjunto das partes de $\Omega$.

Observação $1 \mathbb{P}_{I}$ de fato é uma medida de probabilidade pois:

$$
\mathbb{P}_{I}(\Omega)=\sum_{\omega \in \Omega} \prod_{\substack{\omega_{i}=1 \\ \omega_{j}=-1}} p_{i}\left(1-p_{j}\right)=\prod_{i=1}^{n}\left(p_{i}+\left(1-p_{i}\right)\right)=1
$$

No próximo teorema, através de uma inversão de parâmetros, apresentaremos uma expressão alternativa para (1.7) que servirá de base para incorporarmos uma estrutura de dependências microeconômicas sobre o portfólio.

Teorema 1.7 Considere um portfólio de crédito $\Lambda=\{1,, n\}$ de $n$ tomadores com probabilidades individuais incondicionais de default $\left\{p_{i}\right\}_{i=1}^{n}$ e $\Omega$ o conjunto de estados de default definido em (1.5). Então dado $\omega=\left(\omega_{i}\right)_{i=1}^{n} \in \Omega$, existe $\alpha=\left(\alpha_{i}\right)_{i=1}^{n}, \alpha_{i} \in \mathbb{R}$ tal que:

$$
\mathbb{P}_{I}(\omega)=\frac{1}{Z} e^{\alpha \omega}
$$

onde

$$
Z=\sum_{\omega \in \Omega} e^{\alpha \omega}
$$

com

$$
\alpha \omega=\sum_{i=1}^{n} \alpha_{i} \omega_{i}
$$


se, e somente se, vale a relação:

$$
p_{i}=\frac{1}{1+e^{-2 \alpha_{i}}}
$$

para todo $i=1, . ., n$

Prova: Primeiramente, observe que, para cada $k \in\{1, . ., n\}$, temos

$$
\begin{aligned}
\sum_{\substack{\omega \in \Omega \\
\omega_{k}=+1}} \mathbb{P}_{I}(\omega) & =\sum_{\substack{\omega \in \Omega \\
\omega_{k}=+1}} \prod_{\substack{\omega_{i}=1 \\
\omega_{j}=-1}} p_{i}\left(1-p_{j}\right)= \\
& =\sum_{\substack{\omega \in \Omega \\
\omega_{k}=+1}} \prod_{\substack{\omega_{i}=1 \\
\omega_{j}=-1 \\
i, j \neq k}} p_{k} p_{i}\left(1-p_{j}\right)= \\
& =p_{k} \prod_{\substack{i \neq 1 \\
i \neq k}}^{n}\left(p_{i}+\left(1-p_{i}\right)\right)=p_{k}
\end{aligned}
$$

Portanto, usando (1.13) e escrevendo $\omega=\left(\omega_{i}, \omega^{\prime}\right)$ e $\alpha=\left(\alpha_{i}, \alpha^{\prime}\right)$, onde $\alpha^{\prime}=$ $\left(\alpha_{j}\right)_{j=1, j \neq i}^{n}$ e $\omega^{\prime} \in \Omega^{\prime}$, com

$$
\Omega^{\prime}=\left\{\omega=\left(\omega_{j}\right)_{j=1, j \neq i}^{n}, \omega_{j} \in\{-1,+1\}\right\}
$$

segue que,

$$
\begin{aligned}
& p_{i}=\frac{1}{1+e^{-2 \alpha_{i}}}=\frac{e^{\alpha_{i}}}{e^{+\alpha_{i}}+e^{-\alpha_{i}}} \\
& \Leftrightarrow \quad\left(e^{+\alpha_{i}} \sum_{\omega^{\prime} \in \Omega^{\prime}} e^{\alpha^{\prime} \omega^{\prime}}+e^{-\alpha_{i}} \sum_{\omega^{\prime} \in \Omega^{\prime}} e^{\alpha^{\prime} \omega^{\prime}}\right) p_{i}=e^{\alpha_{i}} \sum_{\omega^{\prime} \in \Omega^{\prime}} e^{\alpha^{\prime} \omega^{\prime}} \\
& \Leftrightarrow\left(\sum_{\substack{\omega \in \Omega \\
\omega i=+1}} e^{\alpha \omega}+\sum_{\substack{\omega \in \Omega \\
\omega i=-1}} e^{\alpha \omega}\right) p_{i}=\sum_{\substack{\omega \in \Omega \\
\omega i=+1}} e^{\alpha \omega} \\
& \Leftrightarrow Z p_{i}=\sum_{\substack{\omega \in \Omega \\
\omega i=+1}} e^{\alpha \omega} \\
& \Leftrightarrow p_{i}=\frac{1}{Z} \sum_{\substack{\omega \in \Omega \\
\omega i=+1}} e^{\alpha \omega}
\end{aligned}
$$


Portanto usando (1.13) e (1.15) obtemos:

$$
\mathbb{P}_{I}(\omega)=\frac{1}{Z} e^{\alpha \omega}
$$

Observe que o modelo probabilístico agora depende do parâmetro $\alpha=$ $\left\{\alpha_{i}\right\}_{i=1}^{n}$ que se relaciona diretamente com as probabilidades incondicionais de default, a saber

$$
p_{i}=\frac{1}{1+e^{-2 \alpha_{i}}}, \quad i=1, . ., n
$$

onde obtemos

$$
\alpha_{i}=-\frac{1}{2} \ln \left(\frac{1}{p_{i}}-1\right), \quad i=1, . ., n
$$

Dizemos então que $\alpha$ é um parâmetro de incondicionalidade já que depende das probabilidades incondicionais de default.

Um outro ponto a ser observado é que a expressão de Z em (1.10) se apresenta como uma soma de ordem exponencial, o que obviamente não é numéricamente conveniente. No presente caso esse problema pode ser resolvido muito facilmente, bastando observar que

$$
Z=\sum_{\omega \in \Omega} e^{\alpha \omega}=\prod_{i=1}^{n}\left(e^{\alpha_{i}}+e^{-\alpha_{i}}\right)=2^{n} \prod_{i=1}^{n} \cosh \alpha_{i}
$$

O teorema 1.7 nos sugere a definição de um modelo mais geral que construiremos na próxima seção. Nesse contexto mais geral o tratamento de somas de ordem exponencial será um problema a ser contornado futuramente.

\subsubsection{Integrando Microestruturas Econômicas}

Nossa meta nessa seção será incorporar no modelo básico apresentado na seção anterior possíveis interações econômicas existentes entre os tomadores do portfólio, respeitando uma estrutura microeconômica fixa, ou seja, iremos supor 
agora que existe dependência entre os eventos de default.

Como comentamos na introdução, nossa motivação para viabilizar esse procedimento é originada em teoria estabelecida na mecânica estatística. Particularmente estamos interessados numa sub-área deste campo de pesquisa onde se estuda o comportamento termodinâmico de materiais ferro-magnéticos. Tais materiais, que podem ser, por exemplo, ferro, níquel ou cobalto, possuem a característica de apresentarem permanente magnetização na ausência de um campo magnético externo e abaixo de uma temperatura crítica chamada temperatura de curie. Acima dessa temperatura crítica esses materiais perdem sua magnetização espontânea. Uma ferramenta essencial para analisar ferro-magnetismo é estabelecida pelo chamado modelo de Ising, onde considera-se um sistema partículas, formada, por exemplo, por átomos ou moléculas, cujo estado (magnetização) se altera conforme o estado de partículas vizinhas. Análogamente ao nosso problema o sistema de partículas é representado considerando-se, essencialmente, um grafo finito $\mathrm{F}$ com $\mathrm{n}$ pontos, onde cada ponto representa um átomo $q_{i}$ que pode assumir somente dois estados. O estado de um átomo representa a sua orientação no campo magnético aqui designado por uma variável $\sigma_{i}$ que pode assumir os valores +1 e -1 (diz-se também que o spin do átomo é positivo ou negativo) e representa-se a interação de energia entre dois átomos $q_{i}$ e $q_{j}$ por uma constante $\tilde{J}$. Particularmente, a ausência de magnetização ou a energia mínima do sistema é caracterizado por todos os spins orientados para baixo ou $\sigma_{i}=-1$ para todo i. A energia total do sistema para uma configuração $\sigma=\left(\sigma_{1},,, \sigma_{N}\right)$ é então representada pelo Hamiltoniano:

$$
\mathcal{H}(\sigma)=-\frac{1}{2} \sum_{(i, j) \in F} \tilde{J} \sigma_{i} \sigma_{j}+M \sum_{i} \sigma_{i}
$$


Onde $M=\left(m_{1},,, m_{N}\right)$ é uma constante representando um campo externo agindo sobre o sistema. As propriedades termodinâmicas do sistema são derivadas da função de partição:

$$
\tilde{Z}=\sum_{\sigma \in F} e^{-\frac{1}{k T} \mathcal{H}(\sigma)}
$$

onde $k$ é conhecido como constante de Boltzmann's e T é temperatura absoluta. O cálculo desta função de partição e em particular o estudo de seu comportamento quando $N \rightarrow \infty$ constitui uma das questões mais relevantes nesse estudo.

No modelo de Ising busca-se, essencialmente, estudar transição de fase do um sistema, onde, em particular, impõe-se condições para se determinar a temperatura crítica do sistema, que é aquela na qual o mesmo deixa de apresentar magnetismo espontâneo, como comentamos inicialmente ${ }^{3}$. Em particular, para medir as configurações de spins do sistema considera-se a função de probabilidade definida por:

$$
\tilde{\mathcal{P}}(\sigma)=\frac{e^{-\frac{1}{k T} \mathcal{H}(\sigma)}}{\tilde{Z}} .
$$

Observando o modelo físico exposto acima fica evidente uma motivação para representarmos interações econômicas entre agentes financeiros conforme apresentamos até aqui ${ }^{4}$ Em nosso caso, no entanto, não pretendemos realizar uma interpretação literal dos conceitos físicos envolvidos no modelo de Ising para nosso contexto econômico mesmo porque nosso objetivo aqui é apenas aprimorar o modelo básico que foi naturalmente desenvolvido na seção anterior. Observe que

\footnotetext{
${ }^{3}$ Para mais detalhes sobre isso veja, por exemplo, [Welsh,1993] e [Uzunov,1992]

${ }^{4} \mathrm{Na}$ verdade uma tentativa de interpretar economicamente o modelo de Ising já foi discutida na literatura científica por Molins e Vives (ver [Molins,2004] ) onde os autores usam esse modelo para estudar os efeitos de correlações ( de natureza puramente macroeconômica) entre os tomadores de um portfólio homogêneo (iguais exposições e probabilidades incondicionais de default) sobre o risco de inadimplência do mesmo. No entanto, as variáveis consideradas no modelo apresentado só estão bem determinadas no limite termodinâmico (ou seja, quando supôe-se que $N \rightarrow \infty$ ) onde, no contexto apresentado, não se obtém resultados realísticos.
} 
a função de probabilidade encontrada em (1.9) é análoga a 1.21 quando $\tilde{J}_{i j}=0$ para todo $i, j$, mostrando uma convergência entre os dois modelos (respeitando os diferentes contextos ). Portanto nos direcionaremos buscando uma representatividade semelhante a utilizada pelo modelo de Ising para estudar ferro-magnetismo dada a semelhança com nosso problema. É importante lembrar também que nosso trabalho está voltado para topologias fixas ( $\mathrm{N}$ finito) enquanto que o modelo de Ising é estudado no limite termodinâmico como vimos cima. Particularmente a motivação para definirmos nosso modelo de incerteza estará voltada para a medida de probabilidade em 1.21, como veremos a partir de agora.

Definição 1.8 Dado um portfólio de crédito $\Lambda=\{1, . ., n\}$ de $n$ tomadores com probabilidades individuais incondicionais de default $\left\{p_{i}\right\}_{i=1}^{n}$ e uma microestrutura econômica $g(\Lambda, \vec{J})$, considere $\Omega$ o conjunto de estados de default sobre $\Lambda$ dado por:

$$
\Omega=\left\{\omega=\left(\omega_{i}\right)_{i=1}^{n}, \omega_{i} \in\{-1,+1\}\right\}
$$

onde:

$$
\omega_{i}=\left\{\begin{array}{llc}
+1 & \text { se } & \text { i default } \\
-1 & \text { se } & \text { i nâ default }
\end{array}\right.
$$

Definimos sobre $\Omega$ o espaço de probabilidade $\left(\Omega, \mathcal{P}(\Omega), \mathbb{P}_{g}\right)$, onde $\mathbb{P}_{g}: \mathcal{P}(\Omega) \mapsto$ $[0,1]$ é definida por:

$$
\mathbb{P}_{g}(\omega)=\frac{1}{Z(g)} e^{\alpha \cdot \omega-\frac{1}{2} \sum_{(i j) \in g} J_{i j} \omega_{i} \omega_{j}}
$$

com

$$
Z(g)=\sum_{\omega \in \Omega} e^{\alpha \cdot \omega-\frac{1}{2} \sum_{(i j) \in g} J_{i j} \omega_{i} \omega_{j}}, \quad \alpha \cdot \omega=\sum_{i=1}^{n} \alpha_{i} \omega_{i} .
$$

sendo $\alpha=\left(\alpha_{i}\right)_{i=1}^{n}$ dado por:

$$
\alpha_{i}=-\frac{1}{2} \ln \left(\frac{1}{p_{i}}-1\right), \quad i=1, . ., n
$$


Observe que um modelo dessa natureza por si só não representa muita utilidade na prática pois em geral é muito difícil observar precisamente como se comportam todos os participantes de um cenário microeconômico. No entanto tal medida de probabilidade será, mais adiante parte integrante de um modelo que reflitirá de forma mais realista um comportamento microestrutural dos tomadores de um portfólio.

Como dissemos inicialmente, agora os estados de default do portfólio correlacionam-se segundo um estrutura microeconômica fixa. Podemos nesse contexto também extrair probabilidades individuais de default do portfólio condicionado a uma determinada estrutura microeconômica, como veremos a seguir.

Definição 1.9 Dado um portfólio de crédito $\Lambda=\{1, n\}$ e uma microestrutura $g(\Lambda, \vec{J})$, temos que a probabilidade individual (condicional) de default de um tomador $i \in \Lambda$ é dada por:

$$
\mathbb{P}_{i}(g(\Lambda, \vec{J}))=\sum_{\substack{\omega \in \Omega \\ \omega_{i}=+1}} \mathbb{P}_{g}(\omega)
$$

onde $\mathbb{P}_{g}: \mathcal{P}(\Omega) \mapsto[0,1]$ é a medida de probabilidade definida em (1.24).

Proposição 1.1 Considere um portfólio de crédito $\Lambda=\{1,,, n\}$ com uma microestrutura $g(\Lambda, \vec{J})$, então temos que a probabilidade individual (condicional) de um tomador $i \in \Omega$ definida em (1.27) é dada por:

$$
\mathbb{P}_{i}(g(\Lambda, \vec{J}))=\frac{1}{1+e^{-2 \alpha_{i}} H(g(\Lambda, \vec{J}))}
$$

com

$$
H(g(\Lambda, \vec{J}))=\frac{\sum_{\omega^{\prime} \in \Omega^{\prime}} e^{H_{2}\left(\omega^{\prime}\right)}}{\sum_{\omega^{\prime} \in \Omega^{\prime}} e^{H_{1}\left(\omega^{\prime}\right)}}
$$


onde, definindo

$$
\Omega^{\prime}=\left\{\omega^{\prime}=\left(\omega_{j}^{\prime}\right)_{j=1}^{n}, \quad \omega_{j}^{\prime} \in\{-1,+1\} \text { se } j \neq i \text { e } \omega_{i}^{\prime}=0\right\}
$$

tem-se que:

$$
\left.H_{1}\left(\omega^{\prime}\right)=\alpha^{\prime} \omega^{\prime}-\frac{1}{2}\left(\sum_{\substack{(i, k) \\ i \neq k}}\left(J_{i k}+J_{k i}\right) \omega_{k}^{\prime}\right)+\sum_{\substack{(k, l) \\ k \neq l \\ k, l \neq i}} J_{k l} \omega_{k}^{\prime} \omega_{l}^{\prime}\right)
$$

e

$$
\left.H_{2}\left(\omega^{\prime}\right)=\alpha^{\prime} \omega^{\prime}+\frac{1}{2}\left(\sum_{\substack{(i, k) \\ i \neq k}}\left(J_{i k}+J_{k i}\right) \omega_{k}^{\prime}\right)-\sum_{\substack{(k, l) \\ k \neq l \\ k, l \neq i}} J_{k l} \omega_{k}^{\prime} \omega_{l}^{\prime}\right)
$$

sendo $\alpha^{\prime}=\left\{\alpha_{j}^{\prime}\right\}_{j=1}^{n} \in \mathbb{R}^{n}$ definido como:

$$
\alpha_{j}^{\prime}=\left\{\begin{array}{ccc}
-\frac{1}{2} \ln \left(\frac{1}{p_{j}}-1\right) & \text { se } j \neq i \\
0 & \text { se } j=i
\end{array}\right.
$$

Prova: Por (1.27) e (1.24) segue que:

$$
\mathbb{P}_{i}(g(\Lambda, \vec{J}))=\sum_{\substack{\omega \in \Omega \\ \omega_{i}=+1}} \mathbb{P}_{g}(\omega)=\frac{1}{Z(g)} \sum_{\substack{\omega \in \Omega \\ \omega_{i}=+1}} e^{\alpha . \omega-\frac{1}{2} \sum_{(i j) \in g} J_{i j} \omega_{i} \omega_{j}}
$$

com

$$
Z(g)=\sum_{\omega \in \Omega} e^{\alpha . \omega-\frac{1}{2} \sum_{(i j) \in g} J_{i j} \omega_{i} \omega_{j}}, \quad \alpha \cdot \omega=\sum_{i=1}^{n} \alpha_{i} \omega_{i} .
$$

sendo $\alpha=\left(\alpha_{j}\right)_{j=1}^{n}$ dado por:

$$
\alpha_{j}=-\frac{1}{2} \ln \left(\frac{1}{p_{j}}-1\right), \quad j=1, . ., n
$$

Definindo o conjunto:

$$
\Omega^{\prime}=\left\{\omega^{\prime}=\left(\omega_{j}^{\prime}\right)_{j=1}^{n}, \omega_{j}^{\prime} \in\{-1,+1\} \text { se } j \neq i \text { e } \omega_{i}^{\prime}=0\right\}
$$

e considerando $\alpha^{\prime}=\left\{\alpha_{j}^{\prime}\right\}_{j=1}^{n} \in \mathbb{R}^{n} \operatorname{com} \alpha_{j}^{\prime}=\alpha_{j}$ para $j \neq i$ e $\alpha_{i}^{\prime}=0$, basta agora observar que:

$$
Z(g)=\sum_{\omega_{\in} \Omega} e^{\alpha \cdot \omega-\frac{1}{2} \sum_{(i j) \in g} J_{i j} \omega_{i} \omega_{j}}=Z_{1}(g)+Z_{2}(g)
$$


onde

$$
\begin{aligned}
& Z_{1}(g)=\sum_{\substack{\omega \in \Omega \\
\omega_{i}=+1}} e^{\alpha \omega-\frac{1}{2} \sum_{(i j) \in g} J_{i j} \omega_{i} \omega_{j}}=e^{\alpha_{i}} \sum_{\omega^{\prime} \in \Omega^{\prime}} e^{H_{1}\left(\omega^{\prime}\right)} \\
& Z_{2}(g)=\sum_{\substack{\omega \in \Omega \\
\omega_{i}=-1}} e^{\alpha \omega-\frac{1}{2} \sum_{(i j) \in g} J_{i j} \omega_{i} \omega_{j}}=e^{-\alpha_{i}} \sum_{\omega^{\prime} \in \Omega^{\prime}} e^{H_{2}\left(\omega^{\prime}\right)}
\end{aligned}
$$

com

$$
\left.H_{1}\left(\omega^{\prime}\right)=\alpha^{\prime} \omega^{\prime}-\frac{1}{2}\left(\sum_{\substack{(i, k) \\ i \neq k}}\left(J_{i k}+J_{k i}\right) \omega_{k}^{\prime}\right)+\sum_{\substack{k, l) \\ k \neq l \\ k, l \neq i}} J_{k l} \omega_{k}^{\prime} \omega_{l}^{\prime}\right)
$$

e

$$
\left.H_{2}\left(\omega^{\prime}\right)=\alpha^{\prime} \omega^{\prime}+\frac{1}{2}\left(\sum_{\substack{(i, k) \\ i \neq k}}\left(J_{i k}+J_{k i}\right) \omega_{k}^{\prime}\right)-\sum_{\substack{k, l) \\ k, \neq \\ k, l \neq i}} J_{k l} \omega_{k}^{\prime} \omega_{l}^{\prime}\right)
$$

Portanto, usando (1.38), (1.39) e(1.40) segue que:

$$
\begin{aligned}
& \mathbb{P}_{i}(g(\Lambda, \vec{J}))=\sum_{\substack{\omega \in \Omega \\
\omega_{i}=+1}} \mathbb{P}_{g}(\omega)=\frac{1}{Z(g)} \sum_{\substack{\omega \in \Omega \\
\omega_{i}=+1}} e^{\alpha \omega-\frac{1}{2} \sum_{(i j) \in g} J_{i j} \omega_{i} \omega_{j}}= \\
& =\frac{Z_{1}(g)}{Z_{1}(g)+Z_{2}(g)}=\frac{1}{1+\frac{Z_{2}(g)}{Z_{1}(g)}}=\frac{1}{1+\frac{e^{-\alpha_{i}} \sum_{\omega^{\prime} \in \Omega^{\prime}} e^{H_{2}\left(\omega^{\prime}\right)}}{e^{\alpha_{i}} \sum_{\omega^{\prime} \in \Omega^{\prime}} e^{H_{1}\left(\omega^{\prime}\right)}}}= \\
& =\frac{1}{1+e^{-2 \alpha_{i}} \frac{\sum_{\omega^{\prime} \in \Omega^{\prime}} e^{H_{2}\left(\omega^{\prime}\right)}}{\sum_{\omega^{\prime} \in \Omega^{\prime}} e^{H_{1}\left(\omega^{\prime}\right)}}}=\frac{1}{1+e^{-2 \alpha_{i}} H(g(\Lambda, \vec{J}))}
\end{aligned}
$$

\subsubsection{Modelo de Incerteza}

Nessa seção finalmente definiremos o modelo de incerteza que nos propusemos a trabalhar. Nesse caso a incerteza residirá sobre todos os estados de default do portfólio considerado sujeito a todas as configurações microestruturais admissíveis. Nesse contexto, ao invés de considerarmos um portfólio sujeito a uma microestrutura fixa de relações microeconômicas, poderemos estudá-lo sugerin- 
do um comportamento microestrutural médio existente entre os tomadores do portfólio de crédito o que na prática é mais fácil de ser observado.

Definição 1.10 (modelo de incerteza)Considere um portfólio de crédito $\Lambda=$ $\{1, . ., n\}$ com probabilidades individuais incondicionais de default, $\left\{p_{i}\right\}_{i=1}^{n}$. Dado uma microestrutura aleatória $G(\Lambda, c, J)$ considere o espaço de estados

$$
\mathcal{N}=\Omega \times G(\Lambda, c, J)=\{\eta=(\omega, g) ; \omega \in \Omega, g \in G(\Lambda, c, J)\}
$$

onde

$$
\Omega=\left\{\omega=\left(\omega_{i}\right)_{i=1}^{n}, \omega_{i} \in\{-1,+1\}\right\} .
$$

satisfaz

$$
\omega_{i}=\left\{\begin{array}{ccc}
+1 & \text { se } & \text { i default } \\
-1 & \text { se } & \text { i não default }
\end{array}\right.
$$

Sobre o conjunto de estados $\mathcal{N}$ definimos o espaço de probabilidade $(\mathcal{N}, \mathcal{P}(\mathcal{N}), \mathbb{P})$ onde a função de probabilidade $\mathbb{P}: \mathcal{P}(\mathcal{N}) \longrightarrow[0,1]$ é dada por:

$$
\mathbb{P}(\eta)=\mathbb{P}(\omega, g)=\mathbb{P}_{g}(\omega) Q(g)
$$

para todo $\eta=(\omega, g) \in \mathcal{N}$, onde $\mathbb{Q}: \mathcal{M} \longrightarrow[0,1]$ é medida de probabilidade definida em 1.4 e, para cada $g \in G(\Lambda, c, J), \mathbb{P}_{g}: \mathcal{P}(\Omega) \mapsto[0,1]$ é definida em $(1.24)$

Também nesse caso podemos determinar as probabilidades individuais de default dos tomadores do portfólio agora estressadas por um cenário microestrutural médio, conforme definimos a seguir.

Definição 1.11 Dado um portfólio de crédito $\Lambda=\{1,,, n\}$ e uma microestrutra aleatória $G(\Lambda, c, J)$, definimos a probabilidade individual de default de um 
tomador $i \in \Lambda$ por:

$$
\tilde{p}_{i}=\sum_{\substack{\eta=(w, g) \in \mathcal{N} \\ w_{i}=+1}} \mathbb{P}(\eta)
$$

Observe que as probabilidades individuais de default de um tomador $i \in \Lambda$ é expresso como uma média sobre as probabilidades individuais (condicionais) de default, pois, para cada $i \in \Lambda$, temos que:

$$
\begin{aligned}
\tilde{p}_{i}=\sum_{\substack{\eta=(\omega, g) \in \mathcal{N} \\
\omega_{i}=+1}} \mathbb{P}(\eta) & =\sum_{\substack{\eta=(\omega, g) \in \mathcal{N} \\
\omega_{i}=+1}} \mathbb{P}_{g}(\omega) Q(g)= \\
& =\sum_{g \in G(\Lambda, c)} \sum_{\substack{\omega \in \Omega \\
\omega_{i}=+1}} \mathbb{P}_{g}(\omega) Q(g) \\
& =\sum_{g \in G(\Lambda, c)} \mathbb{P}_{i}(g) \mathbb{Q}(g)
\end{aligned}
$$

Nosso próximo passo será aplicar nosso modelo no estudo distribuição de perdas de portfólio de crédito. Cumpriremos com esse objetivo utilizando a função geradora da perda que analisaremos na próxima seção.

\subsection{Função Geradora da Perda}

Uma vez definido o modelo de incerteza estudaremos a função geradora de momentos relativo a variável perda de um portfólio. A função geradora é a base de nosso trabalho como ferramenta de trabalho pois é através dela que estudaremos a distribuição de perdas de um portfólio de tomadores de crédito. Nessa seção iremos defini-la e analisá-la num contexto generalizado.

Iremos estudar a função geradora com respeito a uma variável aleatória que descreve as possíveis perdas de um portfólio.

Definição 1.12 Dado um porfólio $\Lambda$ de $n$ tomadores de crédito com exposições $\left\{e_{i}\right\}_{i=1}^{n}$ e uma microestrutura aleatória $G(\Lambda, c, J)$, considere o espaço de probabi- 
lidade $(\mathcal{N}, \mathcal{P}(\mathcal{N}), \mathbb{P})$ construído na definição 1.10. Definimos a perda do portfólio $\Lambda$ como uma variável aleatória $L: \mathcal{N} \mapsto \mathbb{R}^{+}$em $(\mathcal{N}, \mathcal{P}(\mathcal{N}), \mathbb{P})$, definida por:

$$
L(\eta)=\sum_{i=1}^{n} e_{i} \mathbb{L}_{i}(\eta),
$$

onde

$$
\mathbb{L}_{i}(\eta)=\left\{\begin{array}{lll}
1 & \text { se } & \omega_{i}=+1 \\
0 & \text { se } & \omega_{i}=-1
\end{array}\right.
$$

para todo $\eta=(\omega, g) \in \mathcal{N}$.

Definição 1.13 Dada um portfólio de crédito $\Lambda=\{1,,, n\}$ de $n$ tomadores com exposições $\left\{e_{i}\right\}_{i=1}^{n}$ e uma microestrutura eleatória $G(\Lambda, c, J)$, considere a perda

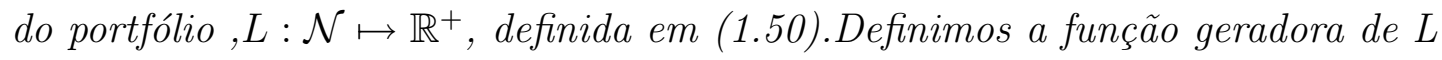
como a função $G: \mathbb{R} \mapsto \mathbb{R}^{+}$dada por:

$$
G(z)=E\left[e^{z L}\right]=\sum_{\eta \in \mathcal{N}} e^{L(\eta)} \mathbb{P}(\eta)
$$

onde E significa a esperança com respeito a perda $L$ e $\mathbb{P}: \mathcal{P}(\mathcal{N}) \longrightarrow[0,1]$ é função de probabilidade definida em (1.47).

A principal propriedade da funçao geradora é que, calculando sucessivamente suas derivadas no ponto $z=0$, obtemos os momentos da perda $L$. Por exemplo, com a primeira derivada obtemos a esperança de L e com a derivada segunda já podemos extrair a variância, como observamos abaixo

$$
G^{\prime}(0)=\left.E\left[L e^{z L}\right]\right|_{z=0}=E[L]
$$

e

$$
G^{\prime \prime}(0)=\left.E\left[L^{2} e^{z L}\right]\right|_{z=0}=E\left[L^{2}\right]
$$


Proposição 1.2 Dada um portfólio de crédito $\Lambda=\{1,,, n\}$ de $n$ tomadores com exposições $\left\{e_{i}\right\}_{i=1}^{n}$ e uma microestrutura eleatória $G(\Lambda, c, J)$ considere a perda do portfólio $L: \mathcal{N} \mapsto \mathbb{R}^{+}$definida em (1.50). Então a função geradora da perda definida em (1.52) é dada por:

$$
G(z)=\sum_{g \in G(\Lambda, c, J)} G_{g}(z) Q(g)
$$

onde $\mathbb{Q}$ é a medida de probabilidade definida em (1.4) e

$$
G_{g}(z)=\frac{e^{z \theta}}{Z(g)} \sum_{\omega \in \Omega} e^{\gamma \omega-\frac{1}{2} \sum_{i j} J_{i j} \omega_{i} \omega_{j}}
$$

com

$$
Z(g)=\sum_{\omega \in \Omega} e^{\alpha \cdot \omega-\frac{1}{2} \sum_{(i j) \in g} J_{i j} \omega_{i} \omega_{j}}, \quad \alpha . \omega=\sum_{i=1}^{n} \alpha_{i} \omega_{i} .
$$

onde, $\theta=\sum_{i=1}^{n} \frac{e_{i}}{2} z$

$\gamma=\left(\gamma_{i}(z)\right)_{i=1}^{n}, \gamma_{i}(z)=\frac{z e_{i}+2 \alpha_{i}}{2}$.

Prova: Primeiramente observemos que:

$$
\begin{aligned}
G(z) & =E\left[e^{z L}\right] \\
& =\sum_{\eta \in \mathcal{N}} e^{L(\eta)} \mathbb{P}(\eta) \\
& =\sum_{g \in G(\Lambda, c, J)} \sum_{\omega \in \Omega} e^{z L(\omega, g)} \mathbb{P}_{g}(\omega) Q(g)
\end{aligned}
$$

portanto, definindo

$$
G_{g}(z)=\sum_{\omega \in \Omega} e^{z L(\omega, g)} \mathbb{P}_{g}(\omega)
$$


segue que

$$
\begin{aligned}
G_{g}(z) & =\sum_{\omega_{\in} \Omega} e^{z L(\omega, g)} \mathbb{P}_{g}(\omega, g)= \\
& =\frac{1}{Z(g)} \sum_{\omega \in \Omega} e^{z L(\omega, g)+\alpha \omega-\frac{1}{2} \sum_{i j} J_{i j} \omega_{i} \omega_{j}}= \\
& =\frac{1}{Z(g)} \sum_{\omega \in \Omega} e^{z \sum_{i=1}^{n} e_{i} \mathbb{I}_{i}(\omega, g)+\sum_{i=1}^{n} \alpha_{i} \omega_{i}-\frac{1}{2} \sum_{i j} J_{i j} \omega_{i} \omega_{j}}= \\
& =\frac{1}{Z(g)} \sum_{\omega \in \Omega} e^{\sum_{i=1}^{n}\left(z e_{i}\left(\frac{1+\omega_{i}}{2}\right)+\alpha_{i} \omega_{i}\right)-\frac{1}{2} \sum_{i j} J_{i j} \omega_{i} \omega_{j}}= \\
& =\frac{e^{\sum_{i=1}^{n} \frac{z e_{i}}{2}}}{Z(g)} \sum_{\omega \in \Omega} e^{\left(\frac{z e_{i}+2 \alpha_{i}}{2}\right) \omega_{i}-\frac{1}{2} \sum_{i j} J_{i j} \omega_{i} \omega_{j}}= \\
& =\frac{e^{z \theta}}{Z(g)} \sum_{\omega \in \Omega} e^{\gamma \omega-\frac{1}{2} \sum_{i j} J_{i j} \omega_{i} \omega_{j}} .
\end{aligned}
$$

onde, $\theta=\sum_{i=1}^{n} \frac{e_{i}}{2} z$

$\gamma=\left(\gamma_{i}(z)\right)_{i=1}^{n}, \gamma_{i}(z)=\frac{z e_{i}+2 \alpha_{i}}{2}$.

onde segue o resultado.

Observação 2 Observe que para o caso de eventos de default independentes $(J=0)$, temos, para cada $g \in G(\Lambda, c, J)$, que:

$$
Z(g)=\sum_{\omega \in \Omega} e^{\alpha \omega}=\prod_{i=1}^{n}\left(e^{\alpha_{i}}+e^{-\alpha_{i}}\right)=2^{n} \prod_{i=1}^{n} \cosh \alpha_{i}
$$

$\log$ :

$$
G_{g}(z)=\frac{e^{z \theta}}{Z(g)} \sum_{\omega \in \Omega} e^{\gamma \omega}=\frac{2^{n} e^{z \theta}}{Z(g)} \prod_{i=1}^{n}\left(e^{\gamma_{i}(z)}+e^{-\gamma_{i}(z)}\right)=e^{\theta z} \prod_{i=1}^{n} \frac{\cosh \gamma_{i}(z)}{\cosh \alpha_{i}}
$$


portanto:

$$
\begin{aligned}
G(z) & =\sum_{g \in G(\Lambda, c, J)} G_{g}(z) Q(g) \\
& =e^{\theta z} \prod_{i=1}^{n} \frac{\cosh \gamma_{i}(z)}{\cosh \alpha_{i}} \sum_{g \in G(\Lambda, c, J)} c^{M(g)}(1-c)^{\left(\begin{array}{c}
n \\
2
\end{array}\right)-M(g)} \\
& =e^{\theta z} \prod_{i=1}^{n} \frac{\cosh \gamma_{i}(z)}{\cosh \alpha_{i}}(c+(1-c))^{n} \\
& =e^{\theta z} \prod_{i=1}^{n} \frac{\cosh \gamma_{i}(z)}{\cosh \alpha_{i}}
\end{aligned}
$$

que é a função geradora para eventos independentes.

Como podemos observar, quando consideramos dependências microeconômicas entre os tomadores do portfólio a expressão da função geradora se torna muito mais complexa e a possibilidade de simplificação direta como no caso independente inexiste. De qualquer modo não é nosso objetivo aqui resolver de forma generalizada a complexidade exponencial que aparece na expressão da função geradora haja vista que nosso meta é investigar cenários microeconômicos particulares, onde este tipo de problema se torna mais tratável. Sendo mais específico ainda, queremos viabilizar um estudo sensibilidade sobre um portfólio de crédito, de forma que estaremos interessados apenas em estudar cenários de contágio microeconômico sugerindo interações microeconômicas de pequena intensidade entre os tomadores. Portanto, sob estas particularidades, veremos no próximo capítulo que podemos obter expressões analiticamente mais aceitáveis para a função geradora da perda que definimos anteriormente o que viabilizará um estudo eficiente da distribuição de perdas de um portfólio de crédito onde existe contágio microeconômico entre seus tomadores. 


\section{Capítulo 2}

\section{Topologias de Contágio}

\subsection{Introdução}

O grande diferencial do modelo probabilístico que construímos no capítulo anterior está no fato dele permitir estudar dependências microeconômicas entre os tomadores de um portfólio de crédito cuja representatividade é dada por estruturas explicitamente estabelecidas. Tal característica nos permite contextualizar o modelo sob diversas topologias de contágio cuja investigação seja pertinente. Evidentemente, num primeiro momento, quando procuramos observar relações econômicas em uma grande economia nos deparamos com complexas teias de interdependências que na prática seria difícil de serem estudadas (explicitamente falando), mesmo considerando uma abordagem estocástica para tais relações. No entanto dentro de toda essa complexidade é possivel identificar cenários típicos cujo estudo pode, sem dúvida, nos fornecer informações importantes sobre o efeito de contágio microeconômico no desempenho de um portfólio de crédito.

Um primeiro cenário em que poderíamos pensar seria de um centro produtivo como mostra a figura1 abaixo, representando a situação de uma firma central interagindo com um conjunto satélite formado por outras firmas com as quais ela mantém relações econômicas diretas. Isto pode ser observado, por exemplo, nas 
relações econômicas que uma grande montadora de automóveis mantém com seus diferentes fornecedores de matéria prima.

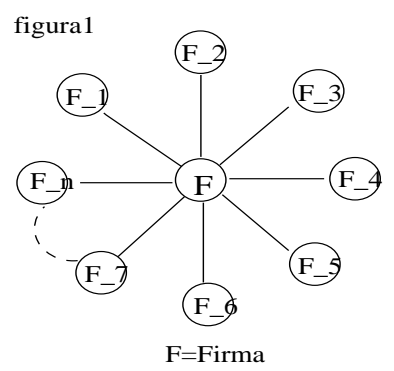

Pode-se estender este cenário considerando uma cadeia produtiva em série como observamos na figura 2. Na figura 3 temos representado o que podemos chamar de uma cadeia produtiva em "cascata", representando por exemplo, uma holding (empresa que mantém o controle sobre outras empresas através da posse majoritária da ações destas) com suas subsidiárias, que sua vez mantém relações econômicas específicas com outras empresas.

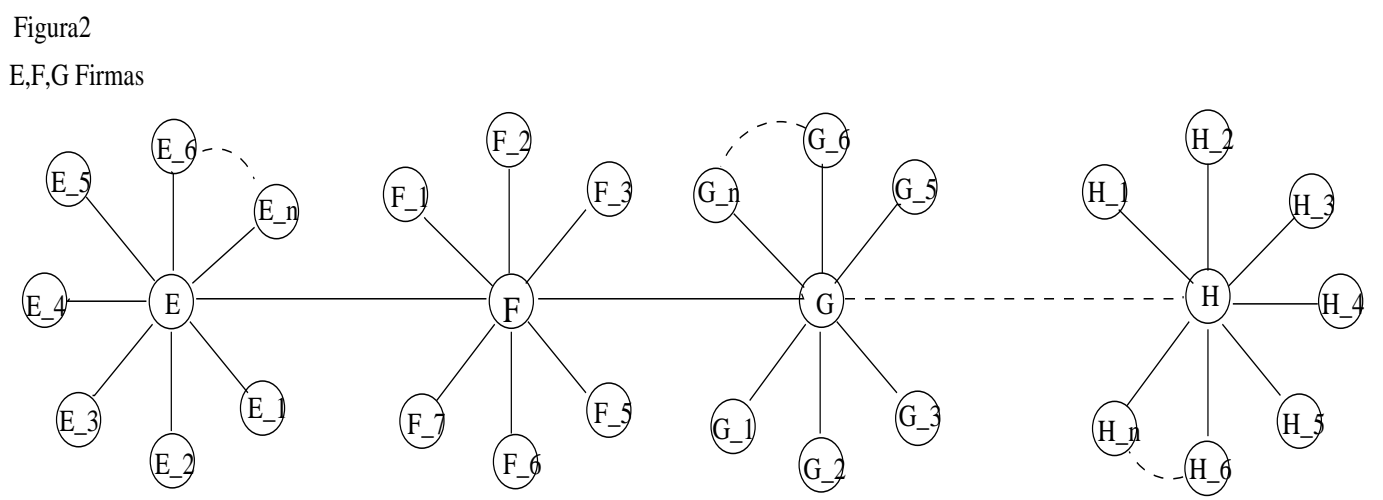




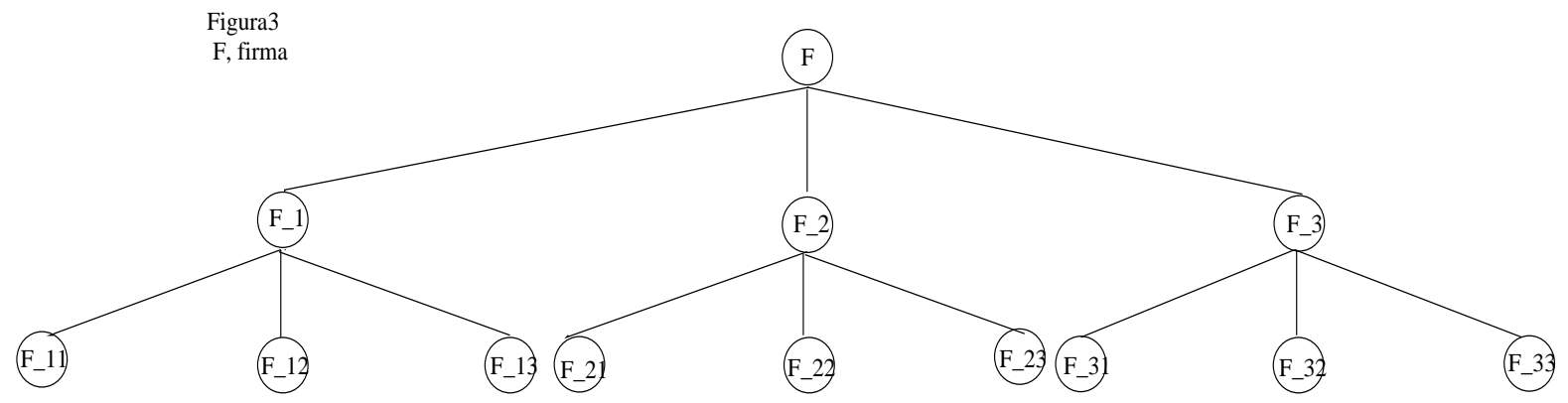

São exatamente cenários dessa natureza que acreditamos ser possível de serem investigados utilizando o modelo proposto. Neste capítulo, particularmente, nos concentraremos em mostrar sua viabilidade no contexto de centros produtivos conforme exemplificado acima. Ficará relativamente claro que para outras variações microestruturais o nível de complexidade para análise é essencialmente equivalente.

Nosso principal objetivo será estimar a distribuição de perdas de um portfólio de crédito cujos tomadores se comportam (microeconômicamente falando) em média, conforme um centro produtivo. Mostraremos que nesse contexto econômico as complexidades exponenciais que caracterizam o modelo em sua versão generalizada aos poucos serão eliminadas, o que mostrará sua viabilidade computacional para análise de sensibilidade a cenários microeconômicos.

\subsection{Centros Produtivos Independentes}

Antes de definirmos formalmente um centro produtivo adotaremos uma notação específica para esse contexto. Cada tomador de crédito aqui será representado por um par $(1, i), i=0, . ., n$ onde o tomador $(1,0)$ representará o centro que interage com um grupo de outros "satélites" do portfólio conforme ilustra a figura abaixo. 


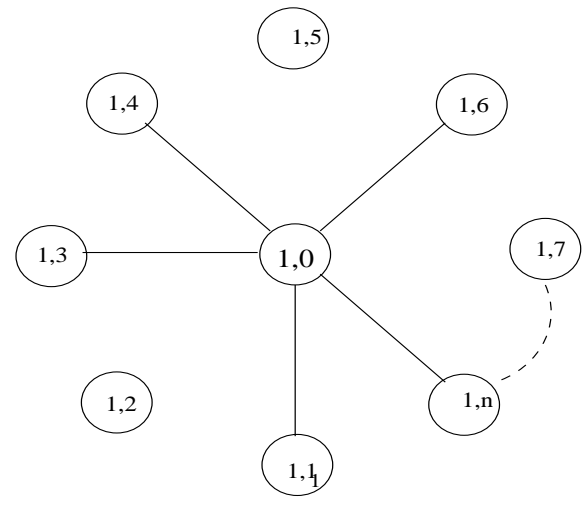

Definição 2.1 Dado um portfólio de crédito $\Lambda=\{(1, i) ; i=0, . ., n\}$, dizemos que uma microestrutura $g(\Lambda, \vec{J})$ é uma microestrutura de centro produtivo, centrado em $(1,0) \in \Lambda$, se $\vec{J}=\left(J_{(1, i)(1, j)}\right)_{i, j}$ satisfaz $J_{(1, i)(1, j)}=0$ para todo $i, j \neq 0$.

Observe na definição acima que só o pode existir interação entre o centro e os satélites mas nunca entre os satélites. Lembremos também que estamos sempre trabalhando com cenários econômicos cujo contágio é uniforme, ou seja:

$$
J_{(1,0)(1, i)}=J \delta_{(1,0)(1, i)}, \quad i=1, . ., n
$$

para algum $J \in \mathbb{R}^{+}$, onde

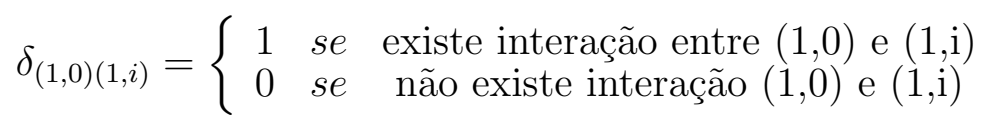

para $i=1, . ., n$.

Observação 3 Daqui em diante sempre que mencionarmos uma microestrutura de centro produtivo já estará implícito a escolha de $(1,0)$ como o centro que interage com outros tomadores do portfólio.

Como em uma microestrutura de centro produtivo o centro não necessariamente interage com todos o outros tomadores do portfólio então podemos de- 
compor o portfólio distinguindo os tomadores independentes do centro e os que interagem com o mesmo conforme enunciamos a seguir.

Proposição 2.1 Dado uma microestrutura de centro produtivo, $g(\Lambda, \vec{J})$, temos que $\Lambda$ admite a seguinte decomposição

$$
\Lambda=\Lambda^{\prime}(g) \cup \Lambda^{\prime \prime}(g) \cup\{(1,0)\}
$$

onde $\Lambda^{\prime}(g)$ e $\Lambda^{\prime \prime}(g)$ são dados por:

$$
\Lambda^{\prime}(g)=\left\{\left(1, i_{l}\right) \in \Lambda ; J_{(1,0)\left(1, i_{l}\right)}=J, l=1, . ., M\right\}
$$

e

$$
\Lambda^{\prime \prime}(g)=\left\{\left(1, j_{k}\right) \in \Lambda ; J_{(1,0)\left(1, j_{k}\right)}=0, k=1, . ., N\right\}
$$

respectivamente, para $0 \leq M, N \leq n$ com $M+N=n$.

Prova: Segue direto da definição.

Ao longo do trabalho será importante representarmos explicitamente a decomposição de um portfólio $\Lambda$ com relação a uma microestrutra de centro produtivo. As notações que adotaremos a seguir irão facilitar esse trabalho.

Notação 2.1 Quando necessário e não houver possibilidade de confusão, usaremos a notação $g\left(\left(i_{l}\right)_{M},\left(j_{k}\right)_{N}, \vec{J}\right)$ para indicar uma microestrutura de centro produtivo com $M$ tomadores, $\left(i_{l}\right)_{M}=\left\{i_{l}\right\}_{l=1}^{M}$, interagindo com o centro $e$ outros $N$ tomadores independentes, $\left(j_{k}\right)_{N}=\left\{j_{k}\right\}_{k=1}^{N}$. Dado um portfólio de crédito $\Lambda=\{1, n\}$, usaremos a identificação:

$$
g(\Lambda, \vec{J}):=g\left(\left(i_{l}\right)_{M},\left(j_{k}\right)_{N}, \vec{J}\right)
$$


para indicar a decomposição de $\Lambda$ sobre g, conforme proposição 3.1.

Quando não houver dúvidas escreveremos simplismente:

$$
g\left(\left(i_{l}\right)_{M},\left(j_{k}\right)_{N}, \vec{J}\right)=g
$$

e indicaremos qualquer aplicação T sobre g (com contágio uniforme J) escrevendo:

$$
T(g)=T\left(g\left(\left(i_{l}\right)_{M},\left(j_{k}\right)_{N}, \vec{J}\right)\right)=T\left(\left(i_{l}\right)_{M},\left(j_{k}\right)_{N}, J\right)
$$

ou ainda

$$
T\left(g\left(\left(i_{l}\right)_{M},\left(j_{k}\right)_{N}, \vec{J}\right)=T\left(\left(i_{l}\right)_{M}, J\right)\right.
$$

$e$

$$
T\left(g\left(\left(i_{l}\right)_{M},\left(j_{k}\right)_{N}, \vec{J}\right)=T\left(\left(j_{k}\right)_{N}, J\right) .\right.
$$

conforme a dependência explicitada por T. Se quisermos explicitar a dependência de $T$ a alguma outra variável também escreveremos:

$$
T(g, z)=T\left(g\left(\left(i_{l}\right)_{M},\left(j_{k}\right)_{N}, \vec{J}\right), z\right)=T\left(\left(j_{k}\right)_{N},\left(j_{k}\right)_{N}, z, J\right)
$$

Notação 2.2 Dado um portfólio de crédito $\Lambda=\{(1, i) ; i=0, . ., n\}$ de $n$ tomadores com exposições $\left(e_{(1, i)}\right)_{i=0}^{n}$ e probabilidades individuais incondicionais de default $\left\{p_{(1, i)}\right\}_{i=0}^{n}$, consideremos $\alpha=\left(\alpha_{(1, i)}\right)_{i=0}^{n}$, onde:

$$
\alpha_{(1, i)}=-\frac{1}{2} \ln \left(\frac{1}{p_{(1, i)}}-1\right), \quad i=0, . ., n .
$$

Usaremos livremente ao longo do texto as funções auxiliares $F_{\left(i_{l}\right)_{M},\left(j_{k}\right)_{N}}: \mathbb{R}^{3} \rightarrow \mathbb{R}$, definidas por:

$$
F_{\left(i_{l}\right)_{M},\left(j_{k}\right)_{N}}(\rho, \sigma, J)=e^{\sigma \alpha_{(1,0)}} \prod_{k=1}^{N} \prod_{l=1}^{M} \cosh \rho \alpha_{\left(1, j_{k}\right)} \cosh \left(\alpha_{\left(1, i_{l}\right)}-\sigma J\right)
$$


Um indice sobre $F_{\left(i_{l}\right)_{M},\left(j_{k}\right)_{N}}$ indica uma restrição sobre o produtório relativo a lista $\left(i_{l}\right)_{M}=\left\{i_{l}\right\}_{l=1}^{M}$, de tomadores dependentes do centro, ou seja,

$$
F_{\left(i_{l}\right)_{M},\left(j_{k}\right)_{N}}^{j}(\rho, \sigma, J)=e^{\sigma \alpha_{(1,0)}} \prod_{k=1}^{N} \prod_{\substack{l=1 \\ i_{l} \neq j}}^{M} \cosh \rho \alpha_{\left(1, j_{k}\right)} \cosh \left(\alpha_{\left(1, i_{l}\right)}-\sigma J\right)
$$

Um indice entre parênteses indica restrição sobre o produtório relativo a lista $\left(j_{k}\right)_{N}$, dos tomadores independentes do centro, ou seja,

$$
F_{\left(i_{l}\right)_{M},\left(j_{k}\right)_{N}}^{(j)}(\rho, \sigma, J)=e^{\sigma \alpha_{(1,0)}} \prod_{\substack{k=1 \\ i_{k} \neq j}}^{N} \prod_{l=1}^{M} \cosh \rho \alpha_{\left(1, j_{k}\right)}(J) \cosh \left(\alpha_{\left(1, i_{l}\right)}(J)-\sigma J\right)
$$

Com as notações acima fica implícito combinações, por exemplo

$$
F_{\left(i_{l}\right)_{M},\left(j_{k}\right)_{N}}^{r, s,(t)}(\rho, \sigma, J)=e^{\sigma \alpha_{(1,0)}} \prod_{\substack{k=1 \\ i_{k} \neq t}}^{N} \prod_{\substack{l=1 \\ i_{l} \neq r, s}}^{M} \cosh \rho \alpha_{\left(1, j_{k}\right)}(J) \cosh \left(\alpha_{\left(1, i_{l}\right)}(J)-\sigma J\right)
$$

Dado $\gamma=\left(\gamma_{\left(1, i_{l}\right)}(z)\right)_{i=1}^{M}, \quad \gamma_{\left(1, i_{l}\right)}(z)=\frac{z e_{\left(1, i_{l}\right)}+2 \alpha_{\left(1, i_{l}\right)}}{2}$, para $z \in \mathbb{R}$, usaremos

também as funções $\tilde{F}_{\left(i_{l}\right)_{M},\left(j_{k}\right)_{N}}$, definidas exatamente como $F_{\left(i_{l}\right)_{M},\left(j_{k}\right)_{N}}$, substituindo $\left(\alpha_{\left(1, i_{l}\right)}\right)_{l=0}^{M}$ por $\left(\gamma_{\left(1, i_{l}\right)}(z)\right)_{l=0}^{M}$ nas expressões, e indicando a dependência pela variável z, por exemplo:

$$
\tilde{F}_{\left(i_{l}\right)_{M},\left(j_{k}\right)_{N}}(\rho, \sigma, z, J)=e^{\sigma \gamma_{(1,0)}(z)} \prod_{k=1}^{N} \prod_{l=1}^{M} \cosh \rho \gamma_{\left(1, j_{k}\right)}(z) \cosh \left(\gamma_{\left(1, i_{l}\right)}(z)-\sigma J\right)
$$

\subsubsection{Modelo de Incerteza}

Nosso próximo passo agora será contextualizar o modelo de incerteza proposto no capítulo anterior nos restringindo a cenários de centro produtivo. Formalmente isso implica em considerar um espaço de probabilidade cujo espaço de eventos é formado por todas as possíveis microestruturas de centro produtivo ( 
com mesmo contágio uniforme), que possam existir a partir de um tomador central fixado. Observe que, nesse caso, considerando um portfólio $\Lambda=\{(1, i) ; i=$ $0, . ., n\}$, existe um número máximo de $\mathrm{n}$ interações possíveis com o centro. Logo a incerteza sobre as estruturas de contágio será modelada por uma medida de probabilidade formada a partir de $\mathrm{n}$ sorteios independentes sobre os pares $((1,0),(1, i))$, $i=1, . ., n$, de tomadores com probabilidade de existência de interação sendo igual a $c \in[0,1]$. Formalmente temos a seguinte definição.

Definição 2.2 Dado um portfólio de crédito $\Lambda=\{(1, i) ; i=0, . ., n\}$, definimos um centro produtivo sobre $\Lambda$ como uma microestrutura aleatória $G(\Lambda, c, J)=$ $(C, \mathcal{F}, \mathbb{Q}), c \in[0,1], J \in \mathbb{R}$, onde $C$ é o espaço de eventos formado por todas as microestuturas de centro produtivo $g(\Lambda, \vec{J})$ com contágio uniforme $J, \mathcal{F}$ é a $\sigma$ - álgebra formada pelos subconjuntos de $C$ e $\mathbb{Q}$ é a medida de probabilidade definida por

$$
\begin{aligned}
\mathbb{Q}: \mathcal{F} & \longrightarrow[0,1] \\
A & \longmapsto \mathbb{Q}(g)=\sum_{g \in A} c^{\left.M_{(} g\right)}(1-c)^{\left.n-M_{(} g\right)}
\end{aligned}
$$

onde $M(g)$ representa o número de interações microeconômicas existentes em $g$.

Assim, dado um portfólio de crédito $\Lambda=\{(1, i) ; i=0,1, n\}$ e um centro produtivo $G(\Lambda, c, J)$ a incerteza sobre o modelo residirá sobre o conjunto de estados

$$
\mathcal{N}=\Omega \times G(\Lambda, c, J)
$$

onde, novamente

$$
\Omega=\left\{\omega=\left(\omega_{(1, i)}\right)_{i=0}^{n}, \omega_{(1, i)} \in\{-1,+1\}\right\} .
$$


com

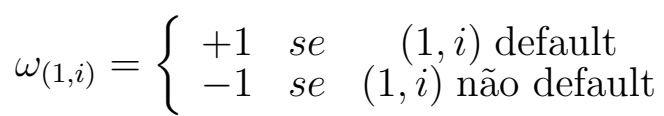

No contexto em que estamos trabalhando, dado uma microestrutura de centro produtivo $g(\Lambda, J)$, podemos também considerar uma decomposição de $\Omega$ sobre $g(\Lambda, J)$ conforme enunciamos a seguir

Definição 2.3 Dado um portfólio de crédito $\Lambda=\{1,,, n\}$ com uma microstrutura de centro produtivo $g(\Lambda, \vec{J})$, e $\Omega$ o espaço de configurações sobre $\Lambda$ definido em (2.20), considere a decomposição $\Lambda=\Lambda^{\prime}(g) \cup \Lambda^{\prime \prime}(g)$ conforme proposição 3.1. Definimos uma decomposição de $\Omega$ sobre $g(\Lambda, \vec{J})$, como:

$$
\Omega=\Omega^{\prime}(g) \cup \Omega^{\prime \prime}(g) \cup\{(1,0)\}
$$

onde

$$
\Omega^{\prime}(g)=\left\{\omega^{\prime}=\left(\omega_{\left(1, i_{l}\right)}\right)_{\left(1, i_{l}\right) \in \Lambda^{\prime}(g)}, \omega_{\left(1, i_{l}\right)} \in\{-1,+1\}\right\}
$$

$e$

$$
\Omega^{\prime \prime}(g)=\left\{\omega^{\prime \prime}=\left(\omega_{\left(1, j_{k}\right)}\right)_{\left(1, j_{k}\right) \in \Lambda^{\prime \prime}(g)}, \omega_{\left(1, j_{k}\right)} \in\{-1,+1\}\right\}
$$

Uma vez contextualizado nosso modelo de incerteza mostraremos agora que podemos obter uma expressão melhorada para a medida de probabilidade definida em (1.47).

Proposição 2.2 Considere um portfólio de crédito $\Lambda=\{(1, i) ; i=0,, n\}$ com probabilidades individuais incondicionais de default $\left\{p_{(1, i)}\right\}_{i=0}^{n}$ e um centro produtivo $G(\Lambda, c, J)$. Então, dado o conjunto de estados $\mathcal{N}$ definido em (2.19), temos que a função de probabilidade $\mathbb{P}: \mathcal{P}(\mathcal{N}) \longrightarrow[0,1]$, definida em (1.47) é dada por:

$$
\mathbb{P}(\eta)=\mathbb{P}_{g}(\omega) Q(g)
$$


para todo $\eta=(\omega, g) \in \mathcal{N}$, onde $Q$ é a medida de probabilidade definida em (2.18) e para todo $g=g\left(\left(i_{l}\right)_{M},\left(j_{k}\right)_{N}, \vec{J}\right) \in G(\Lambda, c, J)$ temos que

$$
\mathbb{P}_{g}(\omega)=\frac{1}{Z(g)} e^{\alpha \omega-J \sum_{l=1}^{M} \omega_{\left(1, i_{l}\right)} \omega_{(1,0)}}
$$

com

$$
Z(g)=2^{n}\left(F_{\left(i_{l}\right)_{M},\left(j_{k}\right)_{N}}(+1,+1, J)+F_{\left(i_{l}\right)_{M},\left(j_{k}\right)_{N}}(+1,-1, J)\right)
$$

para todo $\omega=\left(\omega_{(1, i)}\right)_{i=0}^{n} \in \Omega$, e $\alpha=\left(\alpha_{(1, i)}\right)_{i=0}^{n}$ é dado por:

$$
\alpha_{(1, i)}=-\frac{1}{2} \ln \left(\frac{1}{p_{(1, i)}-1}\right), \quad i=0, . ., n
$$

Prova: Fixando inicialmente uma microestrutura $g\left(\left(i_{l}\right)_{M},\left(j_{k}\right)_{N}, \vec{J}\right)=g$, dado $\omega=\left(\omega_{(1, i)}\right)_{i=0}^{n} \in \Omega$ obtemos:

$$
\begin{aligned}
\mathbb{P}_{g}(\omega) & =\frac{1}{Z(g)} e^{\alpha \omega-\frac{1}{2} \sum_{((1, i),(1, j)) \in g} J_{(1, i)(1, j)} \omega_{(1, i)} \omega_{(1, j)}}= \\
& =\frac{1}{Z(g)} e^{\alpha \omega-\frac{1}{2} \sum_{i=1}^{n}\left(J_{(1,0)(1, i)}+J_{(1, i)(1,0)}\right) \omega_{(1, i)} \omega_{(1,0)}} \\
& =\frac{1}{Z(g)} \cdot e^{\alpha \cdot \omega-J \sum_{l=1}^{M} \omega_{\left(1, i_{l}\right)} \omega_{(1,0)}}
\end{aligned}
$$

onde

$$
\begin{aligned}
Z(g) & =\sum_{\omega \in \Omega} e^{\alpha \omega-\frac{1}{2} \sum_{i=1}^{n}\left(J_{(1,0)(1, i)}+J_{(1, i)(1,0)}\right) \omega_{(1, i)} \omega_{(1,0)}} \\
& =\sum_{\omega^{\prime \prime} \in \Omega^{\prime \prime}} e^{\alpha^{\prime \prime} \omega^{\prime \prime}} \sum_{\omega^{\prime} \in \Omega^{\prime}} e^{\alpha_{(1,0)} \omega_{(1,0)}+\alpha^{\prime} \omega^{\prime}-J \sum_{l=1}^{M} \omega_{(1, i, l} \omega_{(1,0)}}
\end{aligned}
$$

onde $\alpha^{\prime}=\left\{\alpha_{\left(1, i_{l}\right)}\right\}_{l=1}^{M}, \alpha^{\prime \prime}=\left\{\alpha_{\left(1, j_{l}\right)}\right\}_{k=1}^{N}$, e $\Omega^{\prime}, \Omega^{\prime \prime}$ são definidos em (2.23) e (2.24), respectivamente.

Definindo agora:

$$
Z_{1}\left(\left(i_{l}\right)_{M}, J\right)=\frac{1}{2^{M}} \sum_{\omega^{\prime} \in \Omega^{\prime}} e^{\alpha^{\prime} \omega^{\prime}-J \sum_{l=1}^{M} \omega_{\left(1, i_{l}\right)} \omega_{(1,0)}}
$$


e

$$
Z_{2}\left(\left(j_{k}\right)_{N}, J\right)=\frac{1}{2^{N}} \sum_{\omega^{\prime \prime} \in \Omega^{\prime \prime}} e^{\alpha^{\prime \prime} \omega^{\prime \prime}}
$$

segue que:

$$
\begin{aligned}
Z_{2}\left(\left(j_{k}\right)_{N}, J\right) & =\frac{1}{2^{N}} \sum_{\omega^{\prime \prime} \in \Omega^{\prime \prime}} e^{\alpha^{\prime \prime} \omega^{\prime \prime}}= \\
& =\frac{1}{2^{N}} \prod_{l=1}^{N}\left(e^{\alpha_{\left(1, j_{l}\right)}}+e^{\left.-\alpha_{\left(1, j_{l}\right)}\right)}=\right. \\
& =\frac{1}{2^{N}} 2^{N} \prod_{l=1}^{N} \cosh \alpha_{\left(1, j_{l}\right)}= \\
& =\prod_{l=1}^{N} \cosh \alpha_{\left(1, j_{l}\right)} .
\end{aligned}
$$

Por outro lado, definindo o vetor $\tilde{J}=(J,,, J)$ de $\mathrm{M}$ componentes e usando que $\omega_{(1,0)}= \pm 1$, obtemos:

$$
\begin{aligned}
Z_{1}\left(\left(i_{l}\right)_{M}, J\right) & =\frac{1}{2^{M}} \sum_{\omega^{\prime} \in \Omega^{\prime}} e^{\alpha_{(1,0)} \omega_{(1,0)}+\alpha^{\prime} \omega^{\prime}-J \sum_{l=1}^{M} \omega_{\left(1, i_{l}\right)} \omega_{(1,0)}}= \\
& =\frac{1}{2^{M}}\left(\sum_{\omega^{\prime} \in \Omega^{\prime}} e^{\alpha_{(1,0)}+\alpha^{\prime} \omega^{\prime}-J \sum_{l=1}^{M} \omega_{\left(1, i_{l}\right)}}+\sum_{\omega^{\prime} \in \Omega^{\prime}} e^{\left.-\alpha_{(1,0)}+\alpha^{\prime} \omega^{\prime}+J \sum_{l=1}^{M} \omega_{\left(1, i_{l}\right)}\right)=}\right. \\
& =\frac{1}{2^{M}}\left(e^{\alpha_{(1,0)}} \sum_{\omega^{\prime} \in \Omega^{\prime}} e^{\left(\alpha^{\prime}-\tilde{J}\right) \omega^{\prime}}+e^{-\alpha_{(1,0)}} \sum_{\omega^{\prime} \in \Omega^{\prime}} e^{\left(\alpha^{\prime}+\tilde{J}\right) \omega^{\prime}}\right)= \\
& =\frac{1}{2^{M}}\left\{e^{\alpha_{(1,0)}} \prod_{l=1}^{M}\left(e^{\left(\alpha_{\left(1, i_{l}\right)}-J\right)}+e^{-\left(\alpha_{\left(1, i_{l}\right)}-J\right)}\right)+\right. \\
& \left.+e^{-\alpha_{(1,0)}} \prod_{l=1}^{M}\left(e^{\left(\alpha_{\left(1, i_{l}\right.}+J\right)}+e^{-\left(\alpha_{\left(1, i_{l}\right)}+J\right)}\right)\right\}= \\
& =\frac{2^{M}}{2^{M}}\left(e^{\alpha_{(1,0)}} \prod_{l=1}^{M} \cosh \left(\alpha_{\left(1, i_{l}\right)}-J\right)+e^{-\alpha_{(1,0)}} \prod_{i=1}^{M} \cosh \left(\alpha_{\left(1, i_{l}\right.}+J\right)\right)= \\
& \left.=\left(e^{\alpha_{(1,0)}} \prod_{l=1}^{M} \cosh \left(\alpha_{\left(1, i_{l}\right)}-J\right)+e^{-\alpha_{(1,0)}} \prod_{i=1}^{M} \cosh \left(\alpha_{\left(1, i_{l}\right)}\right)+J\right)\right) \cdot(2.34)
\end{aligned}
$$


De (2.33) e (2.34), segue claramente que

$$
\begin{aligned}
Z(g) & =2^{n} Z_{1}\left(\left(i_{l}\right)_{M},\left(j_{k}\right)_{N}, J\right) \cdot Z_{2}\left(\left(j_{k}\right)_{N}, J\right)= \\
& =2^{n}\left(F_{\left(i_{l}\right)_{M},\left(j_{k}\right)_{N}}(+1,+1, J)+F_{\left(i_{l}\right)_{M},\left(j_{k}\right)_{N}}(+1,-1, J)\right) .
\end{aligned}
$$

o que completa a demonstração.

Observe que a restrição do modelo para cenários de centro produtivo melhora bastante a expressão para $\mathbb{P}(\eta)$ em $(2.25)$ já que a complexidade exponencial que reside sobre os estados de default do portfólio foi eliminada, embora a mesma ainda exista sobre as realizações microestruturais. Podemos obter resultados análogos para as probabilidades individuais de default do portfólio como segue:

Proposição 2.3 Dado um portfólio de crédito $\Lambda=\{1,,, n\}$ e um centro produtivo $G(\Lambda, c, J)$ temos que as probabilidades individuais de default definidas em (1.48) são dadas por:

$$
\tilde{p}_{(1,0)}=\sum_{M=0}^{n} \sum_{i_{1}<i_{2}<. .<i_{M}} \mathbb{P}_{(1,0)}\left(\left(i_{l}\right)_{M}, J\right) c^{M}(1-c)^{n-M}
$$

$e$

$$
\tilde{p}_{(1, j)}=\sum_{M=0}^{n} \sum_{\substack{i_{1}<i_{2}<<,<i_{M} \\ j \in\left\{i_{1}, i_{2}, \ldots, i_{M}\right\}}} U\left(J,\left(i_{l}\right)_{M}\right) c^{M}(1-c)^{n-M}+\frac{1}{1+e^{-2 \alpha_{(1, j)}}}
$$

para todo $1 \leq j \leq n$, onde:

$$
\mathbb{P}_{(1,0)}\left(\left(i_{l}\right)_{M}, J\right)=\frac{F_{\left(i_{l}\right)_{M},\left(j_{k}\right)_{N}}(0,+1, J)}{Z_{1}\left(\left(i_{l}\right)_{M}, J\right)}
$$

$e$

$$
U\left(J,\left(i_{l}\right)_{M}\right)=\frac{W\left(J,\left(i_{l}\right)_{M}\right)}{Z_{1}\left(J,\left(i_{l}\right)_{M}\right)}
$$


com

$$
Z_{1}\left(\left(i_{l}\right)_{M}, J\right)=F_{\left(i_{l}\right)_{M},\left(j_{k}\right)_{N}}(0,+1, J)+F_{\left(i_{l}\right)_{M},\left(j_{k}\right)_{N}}(0,-1, J)
$$

$e$

$$
W\left(J,\left(i_{l}\right)_{M}\right)=e^{\alpha_{(1, j)}-J}\left(F_{\left(i_{l}\right)_{M}}^{j}(0,+1, J)+F_{\left(i_{l}\right)_{M}}^{j}(0,-1, J)\right)
$$

As somas em (2.36) e (2.37) são calculadas sobre as decomposições de $\Lambda$ originadas de todas possíveis microestruturas $g\left(\left(i_{l}\right)_{M},\left(j_{k}\right)_{N}, J\right)$ em $G(\Lambda, c, J)$, conforme proposição 2.1.

Prova: Primeiramente observemos que, dado $\eta=(\omega, g) \in \mathcal{N}$, para todo $i=0,1,, n$ tem-se que:

$$
\begin{aligned}
\tilde{p}_{(1, i)}=\sum_{\substack{\eta \in \mathcal{N} \\
\omega(1, i)=+1}} \mathbb{P}(\eta) & =\sum_{\substack{\omega \in \Omega \\
\omega(1, i)=+1}} \sum_{g \in G(\Lambda, c)} \mathbb{P}_{g}(\omega) Q(g)= \\
& =\sum_{g \in G(\Lambda, c)} \sum_{\substack{\omega \in \Omega \\
\omega(1, i)}} \mathbb{P}_{g}(\omega) Q(g) \\
& =\sum_{g \in G(\Lambda, c)} \mathbb{P}_{(1, i)}(g) \mathbb{Q}(g)
\end{aligned}
$$

onde

$$
\mathbb{P}_{(1, i)}(g(\Lambda, \vec{J}))=\sum_{\substack{\omega \in \Omega \\ \omega(1, i)=+1}} \mathbb{P}_{g}(\omega)
$$

com $\mathbb{P}_{g}(\omega)$ sendo dado em $(2.26)$. Portanto primeiro calcularemos $\mathbb{P}_{(1, i)}(g), i=$ $0,,, n$ para uma microestrutura fixada $g(\Lambda, \vec{J})=\left(g\left(\left(i_{l}\right)_{M},\left(j_{k}\right)_{N}, J\right)\right)$. 
Iniciamente observe que

$$
\begin{aligned}
\mathbb{P}_{(1,0)}(g) & =\sum_{\substack{\omega \in \Omega \\
\omega_{(1,0)}=+1}} \mathbb{P}_{g}(\omega)= \\
& =\sum_{\substack{\omega \in \Omega \\
\omega(1,0)=+1}} \frac{1}{Z(g)} e^{\alpha \omega-J \sum_{l=1}^{M} \omega_{\left(1, i_{l}\right)} \omega_{(1,0)}}= \\
& =\frac{e^{\alpha_{(1,0)}}}{Z(g)} \sum_{\omega^{\prime \prime} \in \Omega^{\prime \prime}(g)} e^{\alpha^{\prime \prime} \omega^{\prime \prime}} \sum_{\omega^{\prime} \in \Omega^{\prime}(g)} e^{\left(\alpha^{\prime}-\tilde{J}\right) \omega^{\prime}}
\end{aligned}
$$

$\alpha^{\prime}=\left\{\alpha_{\left(1, i_{l}\right)}\right\}_{l=1}^{M}$ e $\alpha^{\prime \prime}=\left\{\alpha_{\left(1, j_{l}\right)}\right\}_{l=1}^{N}$

Usando (2.31) e (2.30) obtemos:

$$
\begin{aligned}
Z(g) & =\sum_{\omega^{\prime \prime} \in \Omega^{\prime \prime}} e^{\alpha^{\prime \prime} \omega^{\prime \prime}} \sum_{\omega^{\prime} \in \Omega^{\prime}} e^{\alpha^{\prime} \omega^{\prime}-J \sum_{l=1}^{M} \omega_{\left(1, i_{l}\right)} \omega_{(1,0)}}= \\
& =2^{M} Z_{1}\left(\left(i_{l}\right)_{M}, J\right) \sum_{\omega^{\prime \prime} \in \Omega^{\prime \prime}} e^{\alpha^{\prime \prime} \omega^{\prime \prime}}
\end{aligned}
$$

Portanto, segue de (2.44) e (2.45) que

$$
\begin{aligned}
\mathbb{P}_{(1,0)}(g) & =\frac{e^{\alpha_{(1,0)}}}{2^{M} Z_{1}\left(\left(i_{l}\right)_{M}, J\right)} \sum_{\omega^{\prime} \in \Omega^{\prime}} e^{\left(\alpha^{\prime}-\tilde{J}\right) \omega^{\prime}}= \\
& =\frac{2^{M} e^{\alpha_{(1,0)}}}{2^{M} Z_{1}\left(\left(i_{l}\right)_{M}, J\right)} \prod_{l=1}^{M} \cosh \left(\alpha_{\left(1, i_{l}\right)}-J\right)= \\
& =\frac{F_{\left(i_{l}\right)_{M},\left(j_{k}\right)_{N}}(0,+1, J)}{\left.Z_{1}\left(\left(i_{l}\right)_{M}, J\right)\right)}
\end{aligned}
$$

Para o cálculo de $\mathbb{P}_{j}(g), j \in\left\{i_{1}, . ., i_{M}\right\}$, consideremos inicialmente:

$\Omega_{*}^{\prime}(g)=\left\{\left(\omega_{\left(1, i_{l}\right)}\right)_{\left(1, i_{l}\right) \in \Lambda^{\prime}(g), i_{l} \neq j}, \omega_{\left(1, i_{l}\right)} \in\{-1,+1\}\right\}$.

$\alpha_{*}^{\prime}=\left(\alpha_{\left(1, i_{l}\right)}\right)_{\left(1, i_{l}\right) \in \Lambda^{\prime}(g) \backslash\{(1, j)\}}$

$\tilde{J}=(J, . ., J)$, vetor de $M$ componentes.

e

$\tilde{J}_{*}=(J, . ., J)$, vetor de $M-1$ componentes. 
Temos que:

$$
\begin{aligned}
& \mathbb{P}_{(1, j)}(g)=\frac{1}{Z(g)} \sum_{\substack{\omega \in \Omega \\
\omega_{(1, j)}=+1}} e^{\alpha \omega-J \sum_{l=1}^{M} \omega_{\left(1, i_{l}\right)} \omega_{(1,0)}}= \\
& =\frac{1}{Z(g)}\left(\sum_{\substack{\omega \in \Omega \\
\omega(1, j)=+1 \\
\omega(1,0)=+1}} e^{\alpha \omega-J \sum_{l=1}^{M} \omega_{\left(1, i_{l}\right)}}+\sum_{\substack{\omega \in \Omega \\
\omega \in(1, j)=+1 \\
\omega(1,0)=-1}} e^{\left.\alpha \omega-J \sum_{l=1}^{M} \omega_{\left(1, i_{l}\right)}\right)}=\right. \\
& =\frac{1}{Z(g)} \sum_{\omega^{\prime \prime} \in \Omega^{\prime \prime}(g)} e^{\alpha^{\prime \prime} \omega^{\prime \prime}(g)}\left(e^{\alpha_{(1,0)}} \sum_{\substack{\omega^{\prime} \in \Omega^{\prime}(g) \\
\omega_{(1, j)}=+1}} e^{\left(\alpha^{\prime}-\tilde{J}\right) \omega^{\prime}}+\right. \\
& \left.+e^{-\alpha_{(1,0)}} \sum_{\substack{\omega^{\prime} \in \Omega^{\prime}(g) \\
\omega_{(1, j)}=+1}} e^{\left(\alpha^{\prime}+\tilde{J}\right) \omega^{\prime}}\right)= \\
& =\frac{1}{Z(g)} \sum_{\omega^{\prime \prime} \in \Omega^{\prime \prime}(g)} e^{\alpha^{\prime \prime} \omega^{\prime \prime}(g)}\left(e^{\alpha_{(1,0)}+\alpha_{(1, j)}} \sum_{\omega^{\prime} \in \Omega_{*}^{\prime}(g)} e^{\left(\alpha_{*}^{\prime}-\tilde{J}\right) \omega^{\prime}}+\right. \\
& \left.+e^{-\alpha_{(1,0)}+\alpha_{(1, j)}} \sum_{\omega^{\prime} \in \Omega_{*}^{\prime}(g)} e^{\left(\alpha_{*}^{\prime}+\tilde{J}\right) \omega^{\prime}}\right)
\end{aligned}
$$

Usando (2.45), segue de (2.47) que:

$$
\begin{aligned}
\mathbb{P}_{(1, j)} & =\frac{2^{M-1}}{2^{M} Z_{1}\left(\left(i_{l}\right)_{M}, J\right)}\left\{e^{\alpha_{(1,0)}+\alpha_{(1, j)}-J} \prod_{\substack{l=1 \\
i_{l} \neq j}}^{M}\left(e^{\left(\alpha_{\left(1, i_{l}\right)}-J\right)}+e^{-\left(\alpha_{\left(1, i_{l}\right)}-J\right)}\right)+\right. \\
& \left.+e^{-\alpha_{(1,0)}+\alpha_{(1, j)}+J} \prod_{\substack{l=1 \\
i_{l} \neq j}}^{M}\left(e^{\left(\alpha_{\left(1, i_{l}\right.}+J\right)}+e^{-\left(\alpha_{\left(1, i_{l}\right)}+J\right)}\right)\right\}= \\
& =\frac{1}{2 Z_{1}\left(\left(i_{l}\right)_{M}, J\right)}\left\{e^{\alpha_{(1,0)}+\alpha_{(1, j)}-J} \prod_{\substack{l=1 \\
i_{l} \neq j}}^{M} \cosh \left(\alpha_{\left(1, i_{l}\right)}-J\right)+\right. \\
& \left.+e^{-\alpha_{(1,0)}+\alpha_{(1, j)}+J} \prod_{\substack{l=1 \\
i_{l} \neq j}}^{M} \cosh \left(\alpha_{\left(1, i_{l}\right.}+J\right)\right\}= \\
& \left.=\frac{e^{\alpha_{(1, j)}-J}}{2 Z_{1}\left(\left(i_{l}\right)_{M}, J\right)}\left\{F_{\left(i_{l}\right)_{M}}^{j}(0,+1, J)+F_{\left(i_{l}\right)_{M}}^{j}(0,-1, J)\right)\right\}= \\
& =\frac{W\left(J,\left(i_{l}\right)_{M}\right)}{2 Z_{1}\left(\left(i_{l}\right)_{M}, J\right)}
\end{aligned}
$$


Para $j \notin\left\{i_{1}, . ., i_{M}\right\}$, consideremos $\alpha_{*}^{\prime}=\left(\alpha_{\left(1, i_{l}\right)}\right)_{\left(1, i_{l}\right) \in \Lambda^{\prime}(g) \backslash\{(1, j)\}} \mathrm{e}$

$$
\Omega_{*}^{\prime \prime}(g)=\left\{\left(\omega_{\left(1, i_{l}\right)}\right)_{\left(1, i_{l}\right) \in \Lambda^{\prime \prime}(g) \backslash\left\{\left(1, i_{l}\right)\right\}}, \omega_{\left(1, i_{l}\right)} \in\{-1,+1\}\right\}
$$

Temos que

$$
\begin{aligned}
\mathbb{P}_{(1, j)}(g) & =\frac{1}{Z(g)} \sum_{\substack{\omega \in \Omega \\
\omega_{(1, j)}=+1}} e^{\alpha \omega-J \sum_{l=1}^{M} \omega_{\left(1, i_{l}\right)} \omega_{(1, j)}}= \\
& =\frac{1}{Z(g)} \sum_{\substack{\omega^{\prime \prime} \in \Omega^{\prime \prime} \\
\omega_{(1, j)}=+1}} e^{\alpha^{\prime \prime} \omega^{\prime \prime}} \sum_{\omega^{\prime} \in \Omega^{\prime}} e^{\alpha_{(1,0)} \omega_{(1,0)}+\alpha^{\prime} \omega^{\prime}-J \sum_{l=1}^{M} \omega_{\left(1, i_{l}\right)} \omega_{(1,0)}}= \\
& =\frac{e^{\alpha_{(1, j)}}}{Z(g)} \sum_{\omega_{* *}^{\prime \prime} \in \Omega_{* *}^{\prime \prime}} e^{\alpha_{* *}^{\prime \prime} \omega_{* *}^{\prime \prime}} \sum_{\omega^{\prime} \in \Omega^{\prime}} e^{\alpha_{(1,0)} \omega_{(1,0)}+\alpha^{\prime} \omega^{\prime}-J \sum_{l=1}^{M} \omega_{\left(1, i_{l}\right)} \omega_{(1,0)}}
\end{aligned}
$$

Usando (2.30) obtemos:

$$
\begin{aligned}
\mathbb{P}_{(1, j)}(g) & =\frac{e^{\alpha_{(1, j)}}}{\sum_{\omega^{\prime \prime} \in \Omega^{\prime \prime}} e^{\alpha^{\prime \prime} \omega^{\prime \prime}}} \sum_{\omega_{* *}^{\prime \prime} \in \Omega_{* *}^{\prime \prime}} e^{\alpha_{* *}^{\prime \prime} \omega_{* *}^{\prime \prime}}= \\
& =\frac{2^{N-1} e^{\alpha_{(1, j)}}}{\sum_{\omega^{\prime \prime} \in \Omega^{\prime \prime}} e^{\alpha^{\prime \prime} \omega^{\prime \prime}}} \prod_{\substack{l=1 \\
j_{l} \neq j}}^{N}\left(e^{\left.\alpha_{\left(1, j_{l}\right)}\right)}+e^{\left.-\alpha_{\left(1, j_{l}\right)}\right)}\right. \\
& =\frac{2^{N-1} e^{\alpha_{(1, j)}}}{\sum_{\omega^{\prime \prime} \in \Omega^{\prime \prime}} e^{\alpha^{\prime \prime} \omega^{\prime \prime}}} \prod_{\substack{l=1 \\
j_{l} \neq j}}^{N} \cosh \alpha_{\left(1, j_{l}\right)}
\end{aligned}
$$

Como

$$
\begin{aligned}
\sum_{\omega^{\prime \prime} \in \Omega^{\prime \prime}} e^{\alpha^{\prime \prime} \omega^{\prime \prime}} & =\sum_{\substack{\omega^{\prime \prime} \in \Omega^{\prime \prime} \\
\omega_{(1, j)}=+1}} e^{\alpha^{\prime \prime} \omega^{\prime \prime}}+\sum_{\substack{\omega^{\prime \prime} \in \Omega^{\prime \prime} \\
\omega(1, j)=-1}} e^{\alpha^{\prime \prime} \omega^{\prime \prime}}= \\
& =2^{N-1}\left(e^{\alpha_{(1, j)}} \prod_{\substack{l=1 \\
j_{l} \neq j}}^{N} \cosh \alpha_{\left(1, j_{l}\right)}+e^{-\alpha_{(1, j)}} \prod_{\substack{l=1 \\
j_{l} \neq j}}^{N} \cosh \alpha_{\left(1, j_{l}\right)}\right)
\end{aligned}
$$

Segue de (2.50), que

$$
\mathbb{P}_{(1, j)}(g)=\frac{e^{\alpha_{(1, j)}}}{e^{\alpha_{(1, j)}}+e^{-\alpha_{(1, j)}}}=\frac{1}{1+e^{-2 \alpha_{(1, j)}}}
$$


Para o cálculo de $\tilde{p}_{(1,0)}$, considere:

$$
G_{M}(\Lambda, c)=\{g \in G(\Lambda, c): M(g)=M\}, \quad M=0, . ., n
$$

onde $M(g)$ significa o número de interações microeconômicas existentes em g. Dado $\eta=(\omega, g) \in \mathcal{N}$, usando (2.42), obtemos:

$$
\begin{aligned}
\tilde{p}_{(1,0)}(\eta) & =\sum_{g \in G(\Lambda, c)} \mathbb{P}_{(1,0)}(g) \mathbb{Q}(g)= \\
& =\sum_{M=0}^{n} \sum_{g \in G_{M}(n, c)} \mathbb{P}_{(1,0)}(g) c^{M}(1-c)^{n-M}= \\
& =\sum_{M=0}^{n} \sum_{\substack{i_{1}<i_{2}<. .<i_{M} \\
j_{1}<j_{2}<. .<j_{n}-M}} \frac{F_{\left(i_{l}\right)_{M},\left(j_{k}\right)_{N} M}(0,+1, J)}{\left.Z_{1}\left(\left(i_{l}\right)_{M}, J\right)\right)} c^{M}(1-c)^{n-M}
\end{aligned}
$$

Para o cálculo de $\tilde{p}_{(1, j)}$ basta observar que

$$
\begin{aligned}
& \tilde{p}_{(1, j)}=\sum_{g \in G(\Lambda, c)} \mathbb{P}_{(1, j)}(g) \mathbb{Q}(g)= \\
& =\sum_{M=0}^{n} \sum_{i_{1}<i_{2}<. .<i_{M}} \mathbb{P}_{(1, j)}\left(\left(i_{l}\right)_{M}, J\right) c^{M}(1-c)^{M}= \\
& +\sum_{M=0}^{n}\left\{\sum_{\substack{i_{1}<i_{2}<. .<i_{M} \\
j \in\left\{i_{1}, . ., i_{M}\right\}}} \mathbb{P}_{(1, j)}\left(\left(i_{l}\right)_{M}, J\right)+\right. \\
& \left.=\sum_{\substack{M=0 \\
i_{1}<i_{2}<. .<i_{M} \\
j \notin\left\{i_{1}, \ldots, i_{M}\right\}}}^{n} \mathbb{P}_{(1, j)}\left(\left(i_{l}\right)_{M}, J\right)\right\} c^{M}(1-c)^{M} \\
& +\sum_{\substack{i_{1}<i_{2}<.<<i_{M} \\
j \in\left\{i_{1}, i_{2}, \ldots, i_{M}\right\}}} \frac{W\left(J,\left(i_{l}\right)_{M}\right)}{Z_{1}\left(J,\left(i_{l}\right)_{M}\right)}+ \\
& \left.\sum_{\substack{i_{1}<i_{2}<.<<i_{M} \\
j \notin\left\{i_{1}, i_{2}, \ldots, i_{M}\right\}}} \frac{1}{1+e^{-2 \alpha}(1, j)}\right\} c^{M}(1-c)^{n-M}
\end{aligned}
$$


onde $^{1}$

$$
\begin{aligned}
\sum_{M=0}^{n} \sum_{\substack{i_{1}<i_{2}<\ldots<i_{M} \\
j \notin\left\{i_{1}, i_{2}, \ldots, i_{M}\right\}}} c^{M}(1-c)^{n-M} & =\sum_{M=0}^{n-1}\left(\begin{array}{c}
n-1 \\
M
\end{array}\right) c^{M}(1-c)^{n-1-M}= \\
& =(c+(1-c))^{n-1}=1
\end{aligned}
$$

e portanto segue o resultado desejado.

A contextualização do modelo probabilístico feita anteriormente agora nos permitirá analisar a perda de um portfólio onde a incerteza reside sobre os diversos estados de default e as possíveis interações que um tomador central pode estabelecer com os demais tomadores.

Definição 2.4 Dado um porfólio $\Lambda$ de $n$ tomadores de crédito com exposições $\left\{e_{i}\right\}_{i=1}^{n}$ e um centro produtivo $G(\Lambda, c, J)$, considere o espaço de probabilidade $(\mathcal{N}, \mathcal{P}(\mathcal{N}), \mathbb{P})$ onde $\mathbb{P}: \mathcal{P}(\mathcal{N}) \mapsto[0,1]$ é dado em (2.25). Definimos a perda do portfólio $\Lambda$ como uma variável aleatória $L: \mathcal{N} \mapsto \mathbb{R}^{+}$em $(\mathcal{N}, \mathcal{P}(\mathcal{N}), \mathbb{P})$, definida por:

$$
L(\eta)=\sum_{i=1}^{n} e_{(1, i)} \mathbb{L}_{(1, i)}(\eta),
$$

onde

$$
\mathbb{L}_{(1, i)}(\eta)=\left\{\begin{array}{lll}
1 & \text { se } & \omega_{(1, i)}=+1 \\
0 & \text { se } & \omega_{(1, i)}=-1
\end{array}\right.
$$

para todo $\eta=(\omega, g) \in \mathcal{N}$.

Neste contexto a função geradora também assumirá uma expressão melhorada, embora não ainda ideal, como veremos.

Proposição 2.4 Dado um portfólio de crédito $\Lambda=\{(1, i), i=0,, n\}$ com exposições $\left\{e_{(1, i)}\right\}_{i=0}^{n}$ e probabilidades incondicionais de default dadas por $\left\{p_{(1, i)}\right\}_{i=0}^{n}$,

\footnotetext{
${ }^{1}$ observe que para $\mathrm{M}=\mathrm{n}-1$ a soma em questão é igual a zero, já que nesse caso temos $\left\{i_{1}, . ., i_{n}\right\}=\{1, . ., n\} \supset\{j\}$.
} 
considere um centro produtivo $G(\Lambda, c, J)$ e a perda $L: \mathcal{N} \mapsto \mathbb{R}^{+}$no espaço de probabilidade $(\mathcal{N}, \mathcal{P}(\mathcal{N}), \mathbb{P})$ definida em (2.57). Temos que a função geradora de L, definida em (1.52), é dada por:

$$
G(z)=\sum_{M=0}^{n_{1}} \sum_{\substack{i_{1}<i_{2}<. .<i_{M} \\ j_{1}<j_{2}<.<j_{n-M}}} \frac{G\left(\left(i_{l}\right)_{M},\left(j_{k}\right)_{N}, z, J\right)}{Z\left(J,\left(i_{l}\right)_{M},\left(j_{k}\right)_{N}\right)} c^{M}(1-c)^{n-M}
$$

onde

$$
Z\left(J,\left(i_{l}\right)_{M},\left(j_{k}\right)_{N}\right)=2^{n}\left(F_{\left(i_{l}\right)_{M},\left(j_{k}\right)_{N}}(+1,+1, J)+F_{\left(i_{l}\right)_{M},\left(j_{k}\right)_{N}}(+1,-1, J)\right)
$$

$e$

$$
\left.G_{(} z,\left(i_{l}\right)_{M},\left(j_{k}\right)_{N}, \vec{J}\right)=2^{n} e^{z \theta}\left(\tilde{F}_{\left(i_{l}\right)_{M},\left(j_{k}\right)_{N}}(+1,+1, z, J)+\tilde{F}_{\left(i_{l}\right)_{M},\left(j_{k}\right)_{N}}(+1,-1, z, J)\right)
$$

onde

$$
\begin{aligned}
& \theta=\sum_{i=0}^{n} e_{(1, i)} \\
& \gamma=\left(\gamma_{(1, i)}(z)\right)_{i=1}^{n}, \gamma_{(1, i)}(z)=\frac{z e_{(1, i)}+2 \alpha_{(1, i)}}{2}, i=1,2 \ldots, n
\end{aligned}
$$

A soma em (2.59) é calculada sobre as decomposições de $\Lambda$ originadas de todas possiveis microestruturas $g\left(\left(i_{l}\right)_{M},\left(j_{k}\right)_{N}, J\right)$ em $G(\Lambda, c, J)$, conforme proposição 3.1.

Prova: O procedimento é análogo ao cálculo de $Z(g)$ realizado na proposição 2.2, pois $G_{g}(z)$ em (1.56) é dada por:

$$
\begin{aligned}
G_{g}(z) & =\frac{e^{\theta z}}{Z(g)} \sum_{\omega \in \Omega} e^{\gamma \omega} \cdot e^{-\frac{1}{2} \sum_{i=1}^{n}\left(J_{(1,0)(1, i)}+J_{(1, i),(1,0)}\right) \omega_{(1, i)} \omega_{(1,0)}}= \\
& =\frac{e^{\theta z}}{Z(g)} \sum_{\omega \in \Omega} e^{\gamma \omega-J \sum_{l=1}^{M} \omega_{\left(1, i_{l}\right)} \omega_{(1,0)}}
\end{aligned}
$$


que é uma expressão equivalente a $Z(g)$, com diferenças apenas nos parâmetros $\alpha$ e $\gamma$.

Num primeiro momento a função geradora que encontramos em (2.59) não parece muito adequada como ferramenta para estudo da distribuição de perdas de um portfólio de crédito, haja vista que sua expressão se apresenta como uma soma sobre todas as realizações microestruturais possíveis entre os tomadores de um portfólio e cuja complexidade é inaceitável sob ponto de vista computacional. No entanto, mostraremos na próxima seção que tal complexidade pode ser eliminada em um contexto de análise de sensibilidade sobre um portfólio de crédito. Ou seja, sob uma abordagem assintótica para o contágio uniforme das microestruturas encontraremos soluções analíticas de complexidade polinomial para estimar a cauda da distribuição de perdas do portfólio.

\subsubsection{Cálculo da Distribuição de Perdas do Portfólio}

Nessa seção determinaremos uma expressão analítica para a cauda da distribuiçao de perdas de um portfólio de crédito cujo tomadores se relacionam econômicamente conforme um cenário de centro produtivo. No contexto em que construímos nosso modelo de incerteza, a perda considerada se configura conforme os estados de default do portfólio e as diversas configurações microestruturais de centro produtivo que podem existir sobre seus tomadores. Como já dissemos anteriormente, nosso objetivo é viabilizar um estudo de sensibilidade, de forma que nos restringiremos a sugerir interações microeconômicas de pequena intensidade entre os tomadores de crédito e estimar cauda da distribuição de perdas do portfólio conforme sua influência. Obteremos esse resultado realizando um refinamento do método de ponto de sela, uma técnica bem estabelecida no meio 
científico e que vem sendo usada com muita frequência como uma opção viável para determinar expressões analíticas para o VaR de um portfólio de crédito. Segundo Kajai e Skoglund (ver [Kaj,2003]) o método de ponto de sela é extremamente estável para grandes carteiras de exposições o que o torna particularmente muito interessante. Além disso, ainda segundo esses autores, por ser uma aproximação cuja expressão analítica é uma função diferenciável, o método de ponto de sela apresenta outras vantagens do ponto e vista de gerenciamento de risco, como a possibilidade de uma adequada interpretação para o cálculo de risco marginal e contribuição individual de risco.

O principal elemento utilizado pelo método de ponto de sela é a função cumulante que definimos a seguir.

Definição 2.5 Dado um portfólio de crédito $\Lambda=\{(1, i) ; i=0,,, n)\}$ com exposições $\left\{e_{(1, i)}\right\}_{i=0}^{n}$ e probabilidades incondicionais de default dadas por $\left\{p_{(1, i)}\right\}_{i=0}^{n}$, considere um centro produtivo $G(\Lambda, c, J)$ e a perda $L: \mathcal{N} \mapsto \mathbb{R}^{+}$no espaço de probabilidade $(\mathcal{N}, \mathcal{P}(\mathcal{N}), \mathbb{P})$ definida em (2.57). Definimos a função cumulante de $L$ por:

$$
\begin{aligned}
K: & \mathbb{R} \rightarrow \mathbb{R}^{+} \\
& z \rightarrow K(z)=\log G(z)
\end{aligned}
$$

onde G(z) é a função geradora de L dada em (2.59).

Em geral o uso da função cumulante se torna realmente interessante quando considerada sobre variáveis aleatórias independentes, pois nesse caso, ela assume um comportamento aditivo. Em nosso contexto, se assumimos eventos de default 
independentes para os tomadores de um portfólio, obtemos por (1.63), que:

$$
\begin{aligned}
K(z) & =\log \left(e^{\theta z} \prod_{i=0}^{n} \frac{\cosh \gamma_{(1, i)}(z)}{\cosh \alpha_{(1, i)}}\right) \\
& =\theta z+\sum_{i=0}^{n} \log \left(\frac{\cosh \gamma_{(1, i)}(z)}{\cosh \alpha_{(1, i)}}\right)
\end{aligned}
$$

Em geral não se aconselha a trabalhar com o cumulante de variáveis aleatórias dependentes já que nesses casos a obtenção de expressões análogas a (2.66) torna-se bem mais complicada. Em nosso trabalho, apesar de investigarmos dependências entre eventos de default, insistiremos no uso da função cumulante pois mesmo nessas circunstâncias conseguiremos obtê-la sob uma forma analítica de complexidade aceitável.

Pode-se mostrar facilmente que a cauda da probabilidade da perda L definida em (2.57) pode ser calculada pela integral de contorno:

$$
\mathbb{P}(L>t)=\frac{1}{2 \pi i} \int_{-\infty}^{+\infty} \frac{\exp (K(z)-z t)}{z}
$$

cujo caminho de integração reside sob o eixo imaginário. (veja [Kaj,2003] página 51 para uma verificação dessa relação).

O método de ponto de sela consiste em aproximar o termo na exponencial em (2.67) por uma série de taylor ao redor do ponto no qual esse termo é estacionário (o ponto de sela), resultando em uma integral muito mais fácil de ser calculada.

O ponto de sela é portanto, solução da equação:

$$
\frac{d}{d z}(K(z)-z t)=0
$$

Como mencionamos inicialmente o método de ponto de sela é bem conhecido na literatura científica e portanto não entraremos em detalhes sobre ele aqui. Nos limitaremos a enunciar no teorema a seguir sua estimativa para a cauda da 
distribuição de perdas de um porfólio. Para uma demonstraçao completa desse método veja [Kaj,2003] paginas 48 a 51.

Teorema 2.6 (aproximação de ponto de sela) Seja $\mathbb{P}: \mathcal{P}(\mathcal{N}) \rightarrow[0,1]$ a função de probabilidade definida em (2.25) e $K: \mathbb{R} \rightarrow \mathbb{R}^{+}$a função cumulante definida em (2.65). Temos válidos os seguintes resultados:

a) Dado $t \in[0, \infty]$, existe um único $\left.\xi_{t} \in\right]-\infty,+\infty[$, tal que:

$$
\left.\frac{d}{d z} K(z)\right|_{z=\xi_{t}}=t
$$

b) Para $t>E[L]$, onde E[.] significa a esperança sobre L, temos que:

$$
\left.\mathbb{P}(L>t) \simeq \exp \left(K\left(\xi_{t}\right)\right)-t \xi_{t}+\frac{1}{2} \xi_{t}^{2} \frac{d^{2}}{d z^{2}} K\left(\xi_{t}\right)\right) N\left(-\sqrt{\xi_{t}^{2} \frac{d^{2}}{d z^{2}} K\left(\xi_{t}\right)}\right)
$$

onde $N$ é a função de distribuição normal acumulativa.

Observe que a expressão (2.70) não apresenta uma estimativa satisfatória para a cauda da distribuição de perdas do portfólio pois a função cumulante e suas derivadas apresentam complexidade exponencial em suas expressões já que são definidas em termos da função geradora da perda. O próximo teorema resolverá esse problema demonstrando que as somas exponencias podem ser eliminadas se restringirmos nosso estudo para uma vizinhança de $J=0$.

Para efeito de auxilio para ao teorema que enunciaremos adiante, dado $z \in$ $\mathbb{R}$, considere os seguintes grupos de funções: 
A)

$$
\begin{aligned}
X^{(0)}(z) & =z \theta+\sum_{i=0}^{n} \log \left(\frac{\cosh \gamma_{(1, i)}(z, 0)}{\cosh \alpha_{(1, i)}(0)}\right) \\
X^{(1)}(z) & =c \sum_{r=1}^{n}\left(\operatorname{tgh} \gamma_{(1,0)} \operatorname{tgh} \gamma_{(1, r)}(z)-\operatorname{tgh} \alpha_{(1,0)} \operatorname{tgh} \alpha_{(1, r)}\right) \\
X^{(2)}(z) & =X_{1}^{(2)}(z)+X_{2}^{(2)}(z) \\
X_{1}^{(2)}(z) & =\left(X^{(1)}(z)\right)^{2} \\
X_{2}^{(2)}(z) & =X_{2,1}^{(2)}(z)+X_{2,2}^{(2)}(z) \\
X_{2,1}^{(2)}(z) & =\sum_{r=1}^{n} c\left\{1+\operatorname{ctgh} \gamma_{(1, r)}(z) \sum_{\substack{s=1 \\
s \neq r}} \operatorname{tgh} \gamma_{(1, s)}(z)\right\}+ \\
& +c^{2}\left(\operatorname{tgh} \alpha_{(1,0)} \sum_{r=1}^{n} \operatorname{tgh} \alpha_{(1, r)}\right)\left(\operatorname{tgh} \gamma_{(1,0)}(z) \sum_{t=1}^{n} \operatorname{tgh} \gamma_{1, t}(z)\right) \\
X_{2,2}^{(2)}(z) & =c^{2}\left(\operatorname{tgh} \alpha_{(1,0)} \sum_{r=1}^{n} \operatorname{tgh} \alpha_{(1, r)}\right)\left(\operatorname{tgh} \gamma_{(1,0)}(z) \sum_{t=1}^{n} \operatorname{tgh} \gamma_{1, t}(z)\right) \\
& +\sum_{r=1}^{n} c\left\{1+c t g h \alpha_{(1, r)} \sum_{\substack{s=1 \\
s \neq r}}^{n} \operatorname{tgh} \alpha_{(1, s)}\right\}+ \\
& \left.+\left(2 c t g h \alpha_{(1,0)}\right) \sum_{r=1}^{n} \operatorname{tgh} \alpha_{(1, r)}\right)^{2} .
\end{aligned}
$$

B)

$$
\begin{aligned}
Y^{(0)}(z) & =\theta+\frac{1}{2} \sum_{i=0}^{n} e_{(1, i)} \operatorname{tgh} \gamma_{(1, i)}(z, 0) \\
Y^{(1)}(z) & =Y_{1}^{(1)}(z)+Y_{2}^{(1)}(z) \\
Y^{(2)}(z) & =Y_{1}^{(2)}(z)+Y_{2}^{(2)}(z) \\
Y_{1}^{(1)}(z) & =\frac{c}{2} e_{(1,0)} \sum_{r=1}^{n} \operatorname{sech}^{2}\left(\gamma_{(1,0)}(z)\right) \tanh \left(\gamma_{(1, r)}(z)\right) \\
Y_{2}^{(1)}(z) & =\frac{c}{2} e_{(1,0)} \sum_{r=1}^{n} \operatorname{sech}^{2}\left(\gamma_{(1, r)}(z)\right) \tanh \left(\gamma_{(1,0)}(z)\right) e_{(1, r)}
\end{aligned}
$$


$\left.B_{1}\right)$

$$
\begin{aligned}
& Y_{1}^{(2)}(z)=Y_{1,1}^{(2)}(z)+Y_{1,2}^{(2)}(z) \\
& Y_{1,1}^{(2)}(z)=Y_{1,1,1}^{(2)}(z)+Y_{1,1,2}^{(2)}(z) \\
& Y_{1,2}^{(2)}(z)=Y_{1,1,2}^{(2)}(z) \\
& Y_{1,1,1}^{(2)}(z)=Y_{1,1,1,1}^{(2)}+Y_{1,1,1,2}^{(2)} \\
& Y_{1,1,1,1}^{(2)}(z)=c^{2} \sum_{r=1}^{n}\left[\sum_{\substack{s=1 \\
s \neq r}}^{n} \operatorname{sech}^{2}\left(\gamma_{(1, r)}(z)\right) e_{(1, r)} \operatorname{tgh}\left(\gamma_{(1, r)}(z)\right)\right] \\
& Y_{1,1,1,2}^{(2)}(z)=c^{2} \sum_{r=1}^{n}\left[\sum_{\substack{s=1 \\
s \neq r}}^{n} \operatorname{tgh}\left(\gamma_{(1, r)}(z)\right) \operatorname{sech}^{2}\left(\gamma_{(1, s)}(z)\right) e_{(1, s)}\right] \\
& Y_{1,1,2}^{(2)}(z)=Y_{1,1,2,1}^{(2)}(z)+Y_{1,1,2,2}^{(2)}(z) \\
& Y_{1,1,2,1}^{(2)}(z)=\frac{c^{2} e_{(1,0)}}{2}\left(\sum_{r=1}^{n} \operatorname{tgh}\left(\alpha_{(1,0)}\right) \operatorname{tgh}\left(\alpha_{(1, r)}\right)\right) \times \\
& \times\left(\sum_{r=1}^{n} \operatorname{sech}^{2}\left(\gamma_{(1,0)}(z)\right) \operatorname{tgh}\left(\gamma_{(1, r)}(z)\right)\right) \\
& Y_{1,1,2,2}^{(2)}(z)=\frac{c^{2}}{2}\left(\sum_{r=1}^{n} \operatorname{tgh}\left(\alpha_{(1,0)}\right) \operatorname{tgh}\left(\alpha_{(1, r)}\right)\right) \times \\
& \times\left(\sum_{r=1}^{n} \operatorname{sech}^{2}\left(\gamma_{(1, r)}(z)\right) \operatorname{tgh}\left(\gamma_{(1,0)}(z)\right)\right)
\end{aligned}
$$

$\left.B_{2}\right)$

$$
\begin{aligned}
Y_{2}^{(2)}(z) & =Y_{2,1}^{(2)}(z)+Y_{2,2}^{(2)}(z)+Y_{2,3}^{(2)}(z)+Y_{2,4}^{(2)}(z) \\
Y_{2,1}^{(2)}(z) & =7 c^{2} e_{(1,0)}\left(\sum_{r=1}^{n} \operatorname{tgh}\left(\gamma_{(1, r)}(z)\right) \operatorname{tgh}\left(\gamma_{(1,0)}(z)\right)\right) \times \\
& \times\left(\sum_{r=1}^{n} \operatorname{sech}^{2}\left(\gamma_{(1,0)}(z)\right) \operatorname{tgh}\left(\gamma_{(1, r)}(z)\right)\right)
\end{aligned}
$$




$$
\begin{aligned}
Y_{2,2}^{(2)}(z) & =c^{2}\left(\sum_{r=1}^{n} \operatorname{tgh}\left(\gamma_{(1, r)}(z)\right) \operatorname{tgh}\left(\gamma_{(1,0)}(z)\right)\right) \times \\
& \times\left(\sum_{r=1}^{n} \operatorname{sech}^{2}\left(\gamma_{(1, r)}(z)\right) \operatorname{tgh}\left(\gamma_{(1,0)}(z)\right) e_{(1, r)}\right) \\
Y_{2,3}^{(2)}(z) & =-\frac{c^{2}}{2}\left(\sum_{r=1}^{n} \operatorname{tgh}\left(\alpha_{(1, r)}\right) \operatorname{tgh}\left(\alpha_{(1,0)}\right)\right) \times \\
& \times\left(\sum_{r=1}^{n} \operatorname{sech}^{2}\left(\gamma_{(1,0)}(z)\right) \operatorname{tgh}\left(\gamma_{(1, r)}(z)\right) e_{(1,0)}\right) \\
Y_{2,4}^{(2)}(z) & =-\frac{c^{2}}{2} e_{(1,0)}\left(\sum_{r=1}^{n} \operatorname{tgh}\left(\alpha_{(1,0)}\right) \operatorname{tgh}\left(\alpha_{(1, r)}\right)\right) \times \\
& \times\left(\sum_{r=1}^{n} \operatorname{sech}^{2}\left(\gamma_{(1, r)}(z)\right) \operatorname{tgh}\left(\gamma_{(1,0)}(z)\right) e_{(1, r)}\right)
\end{aligned}
$$

C)

$$
\begin{aligned}
& Z^{(0)}(z)=\frac{1}{4} \sum_{i=0}^{n} e_{(1, i)}^{2}\left(1-\operatorname{tgh} \gamma_{(1, i)}^{2}(z, 0)\right) \\
& Z^{(1)}(z)=Z_{1}^{(1)}(z)+Z_{2}^{(1)}(z)+Z_{3}^{(1)}(z)+Z_{4}^{(1)}(z) \\
& Z^{(2)}(z)=Z_{1}^{(2)}(z)+Z_{2}^{(2)}(z) \\
& Z_{1}^{(1)}(z)=\frac{c}{4} \sum_{r=1}^{n} \operatorname{sech}^{2}\left(\gamma_{(1,0)}(z)\right) \operatorname{sech}^{2}\left(\gamma_{(1, r)}(z)\right) e_{(1,0)} e_{(1, r)} \\
& Z_{2}^{(1)}(z)=-\frac{c}{2} \sum_{r=1}^{n} \operatorname{sech}^{2}\left(\gamma_{(1,0)}(z)\right) \operatorname{tgh}\left(\gamma_{(1,0)}(z)\right) \operatorname{tgh}\left(\gamma_{(1, r)}(z)\right) e_{(1,0)}^{2} \\
& Z_{3}^{(1)}(z)=\frac{c}{4} \sum_{r=1}^{n} \operatorname{sech}^{2}\left(\gamma_{(1,0)}(z)\right) \operatorname{sech}^{2}\left(\gamma_{(1, r)}(z)\right) e_{(1,0)} e_{(1, r)} \\
& Z_{4}^{(1)}(z)=-\frac{c}{2} \sum_{r=1}^{n} \operatorname{sech}^{2}\left(\gamma_{(1, r)}(z)\right) \operatorname{tgh}\left(\gamma_{(1,0)}(z)\right) \operatorname{tgh}\left(\gamma_{(1, r)}(z)\right) e_{(1, r)}^{2}
\end{aligned}
$$




$$
\begin{aligned}
& Z_{1}^{(2)}(z)=Z_{1,1}^{(2)}(z)+Z_{1,2}^{(2)}(z) \\
& Z_{1,1}^{(2)}(z)=Z_{1,1,1}^{(2)}(z)+Z_{1,1,2}^{(2)}(z) \\
& Z_{1,2}^{(2)}(z)=Z_{1,1,2}^{(2)}(z) \\
& Z_{1,1,1}^{(2)}(z)=Z_{1,1,1,1}^{(2)}(z)+Z_{1,1,1,2}^{(2)}(z)+Z_{1,1,1,3}^{(2)}(z) \\
& Z_{1,1,1,1}^{(2)}(z)=c^{2} \sum_{r=1}\left[\frac{1}{2} \operatorname{sech}^{4}\left(\gamma_{(1, r)}(z)\right)-\operatorname{sech}^{2}\left(\gamma_{(1, r)}(z)\right) \operatorname{tgh}^{2}\left(\gamma_{(1, r)}(z)\right)\right] e_{(1, r)}^{2} \\
& Z_{1,1,1,2}^{(2)}(z)=\frac{c^{2}}{2} \sum_{r=1}^{n} \sum_{\substack{s=1 \\
s \neq r}}^{n} \operatorname{sech}^{2}\left(\gamma_{(1, s)}(z)\right) \operatorname{sch}^{2}\left(\gamma_{(1, r)}(z)\right) e_{(1, r)} e_{(1, s)} \\
& Z_{1,1,1,3}^{(2)}(z)=-c^{2} \sum_{r=1}^{n} \sum_{\substack{s=1 \\
s \neq r}}^{n} \operatorname{sech}^{2}\left(\gamma_{(1, s)}(z)\right) \operatorname{tgh}\left(\gamma_{(1, r)}(z)\right) \operatorname{tgh}\left(\gamma_{(1, s)}(z)\right) e_{(1, s)}^{2} \\
& Z_{1,1,2}^{(2)}=Z_{1,1,2,1}^{(2)}+Z_{1,1,2,2}^{(2)} \\
& Z_{1,1,2,1}^{(2)}=\frac{c^{2} e_{(1,0)} e_{(1, r)}}{4}\left(\sum_{r=1}^{n} \operatorname{tgh}\left(\alpha_{(1,0)}\right) \operatorname{tgh}\left(\alpha_{(1, r)}\right)\right) \times \\
& \times\left(\sum_{r=1}^{n} \operatorname{sech}^{2}\left(\gamma_{(1,0)}(z)\right) \operatorname{sech}^{2}\left(\gamma_{(1, r)}\right)\right) \\
& Z_{1,1,2,2}^{(2)}=-\frac{c^{2}}{2}\left(\sum_{r=1}^{n} \operatorname{tgh}\left(\alpha_{(1,0)}\right) \operatorname{tgh}\left(\alpha_{(1, r)}\right)\right) \times \\
& \times\left(\sum_{r=1}^{n} \operatorname{sech}^{2}\left(\gamma_{(1,0)}(z)\right) \operatorname{tgh}\left(\gamma_{(1, r)}\right) \operatorname{tgh}\left(\gamma_{(1,0)}(z)\right)\right. \\
& Z_{2}^{(2)}(z)=Z_{2,1}^{(2)}(z)+Z_{2,2}^{(2)}(z)+Z_{2,3}^{(2)}(z) \\
& Z_{2,1}^{(2)}(z)=Z_{2,1,1}^{(2)}(z)+Z_{2,1,2}^{(2)}(z)+Z_{2,1,3}^{(2)}(z) \\
& Z_{2,1,1}^{(2)}(z)=\frac{c^{2} e_{(1,0)}^{2}}{2} \sum_{r=1}^{n} \sum_{\substack{s=1 \\
s \neq r}}^{n} \operatorname{sech}^{2}\left(\gamma_{(1,0)}(z)\right) \operatorname{tgh}\left(\gamma_{(1, r)}(z)\right) \operatorname{tgh}\left(\gamma_{(1, s)}(z)\right) \\
& Z_{2,1,2}^{(2)}(z)=c^{2} e_{(1,0)} \sum_{r=1}^{n} \operatorname{sech}^{2}\left(\gamma_{(1,0)}(z)\right) \operatorname{sech}^{2}\left(\gamma_{(1, r)}(z)\right) \operatorname{tgh}\left(\gamma_{(1,0)}(z)\right) \times \\
& \times \operatorname{tgh}\left(\gamma_{(1, r)}(z)\right) e_{(1, r)} \\
& Z_{2,1,3}^{(2)}(z)=-c^{2} e_{(1,0)}^{2} \sum_{r=1}^{n} \operatorname{sech}^{2}\left(\gamma_{(1, \not, \mathcal{Z}}(z)\right) \operatorname{tgh}^{2}\left(\gamma_{(1,0)}(z)\right) \operatorname{tgh}^{2}\left(\gamma_{(1, r)}(z)\right)
\end{aligned}
$$




$$
\begin{aligned}
& Z_{2,2}^{(2)}(z)=Z_{2,2,1}^{(2)}(z)+Z_{2,2,2}^{(2)}(z)+Z_{2,3,3}^{(2)}(z) \\
& Z_{2,2,1}^{(2)}(z)=\frac{c^{2}}{2} \sum_{r=1}^{n} \sum_{\substack{s=1 \\
s \neq r}}^{n} \operatorname{sech}^{2}\left(\gamma_{(1, r)}(z)\right) \operatorname{tgh}\left(\gamma_{(1,0)}(z)\right) \operatorname{tgh}\left(\gamma_{(1, s)}(z)\right) \\
& Z_{2,2,2}^{(2)}(z)=c^{2} e_{(1,0)} \sum_{r=1}^{n} \operatorname{sech}^{2}\left(\gamma_{(1,0)}(z)\right) \operatorname{sech}^{2}\left(\gamma_{(1, r)}(z)\right) \operatorname{tgh}\left(\gamma_{(1,0)}(z)\right) \times \\
& \times \operatorname{tgh}\left(\gamma_{(1, r)}(z)\right) e_{(1, r)} \\
& Z_{2,1,3}^{(2)}(z)=-c^{2} \sum_{r=1}^{n} \operatorname{sech}^{2}\left(\gamma_{(1, r)}(z)\right) \operatorname{tgh}^{2}\left(\gamma_{(1,0)}(z)\right) \operatorname{tgh}^{2}\left(\gamma_{(1, r)}(z)\right) e_{(1, r)}^{2} \\
& Z_{2,3}^{(2)}(z)=Z_{2,3,1}^{(2)}(z)+Z_{2,3,2}^{(2)}(z) \\
& Z_{2,3,1}^{(2)}(z)=-\frac{c^{2} e_{(1,0)}}{4}\left(\sum_{r=1}^{n} \operatorname{sech}^{2}\left(\gamma_{(1,0)}(z)\right) \operatorname{sech}^{2}\left(\gamma_{(1, r)}(z)\right) e_{(1, r)}\right) \times \\
& \times \quad\left(\operatorname{tgh}\left(\alpha_{(1,0)}(z)\right) \operatorname{tgh}\left(\alpha_{(1, r)}\right)\right) \\
& Z_{2,3,2}^{(2)}(z)=\frac{c^{2} e_{(1,0)}}{2} \sum_{r=1}^{n}\left(\operatorname{sech}^{2}\left(\gamma_{(1,0)}(z)\right) \operatorname{tgh}\left(\gamma_{(1, r)}(z)\right) \operatorname{tgh}\left(\gamma_{(1,0)}\right)\right) \times \\
& \times\left(\operatorname{tgh}\left(\alpha_{(1,0)}\right) \operatorname{tgh}\left(\alpha_{(1, r)}\right)\right) \\
& Z_{2,4}^{(2)}(z)=Z_{2,4,1}^{(2)}(z)+Z_{2,4,2}^{(2)}(z) \\
& Z_{2,4,1}^{(2)}(z)=-\frac{c^{2} e_{(1,0)}}{4}\left(\sum_{r=1}^{n} \operatorname{sech}^{2}\left(\gamma_{(1,0)}(z)\right) \operatorname{sech}^{2}\left(\gamma_{(1, r)}(z)\right) e_{(1, r)}\right) \times \\
& \times \quad\left(\operatorname{tgh}\left(\alpha_{(1,0)}\right) \operatorname{tgh}\left(\alpha_{(1, r)}\right)\right) \\
& Z_{2,4,2}^{(2)}(z)=\frac{c^{2} e_{(1,0)}}{2} \sum_{r=1}^{n}\left(\operatorname{sech}^{2}\left(\gamma_{(1, r)}(z)\right) \operatorname{tgh}\left(\gamma_{(1, r)}(z)\right) \operatorname{tgh}\left(\gamma_{(1,0)}\right)\right) \times \\
& \times \quad\left(\operatorname{tgh}\left(\alpha_{(1,0)}\right) \operatorname{tgh}\left(\alpha_{(1, r)}\right)\right)
\end{aligned}
$$

Para auxiliar os cálculos a serem feitos também enunciaremos a seguir as seguintes identidades,já conhecidas, com relação a função geradora da perda dada 
em (2.59):

$$
G(z)=\sum_{M=0}^{n} \sum_{\substack{i_{1}<i_{2}<. .<i_{M} \\ j_{1}<j_{2}<.<j_{n-M}}} G\left(z,\left(i_{l}\right)_{M},\left(j_{k}\right)_{N}, \vec{J}\right) c^{M}(1-c)^{n-M}
$$

Temos então as seguintes identidades:

$$
\begin{aligned}
G\left(\left(i_{l}\right)_{M},\left(j_{k}\right)_{N}, \vec{J}\right) & =e^{z \theta} \tilde{G}\left(z,\left(i_{l}\right)_{M},\left(j_{k}\right)_{N}, \vec{J}\right) \\
\tilde{G}\left(z,\left(i_{l}\right)_{M},\left(j_{k}\right)_{N}, \vec{J}\right) & =\frac{H\left(\left(i_{l}\right)_{M},\left(j_{k}\right)_{N}, J\right)}{Z\left(J,\left(i_{l}\right)_{M},\left(j_{k}\right)_{N}\right)} \\
H\left(\left(i_{l}\right)_{M},\left(j_{k}\right)_{N}, z, J\right) & =\tilde{F}_{\left(i_{l}\right)_{M},\left(j_{k}\right)_{N}}(+1,+1, z, J)+ \\
& +\tilde{F}_{\left(i_{l}\right)_{M},\left(j_{k}\right)_{N}}(+1,-1, z, J) \\
Z\left(J,\left(i_{l}\right)_{M},\left(j_{k}\right)_{N}\right) & =F_{\left(i_{l}\right)_{M},\left(j_{k}\right)_{N}}(+1,+1, J)+ \\
& +F_{\left(i_{l}\right)_{M},\left(j_{k}\right)_{N}(+1,-1, J)}^{N} \\
F_{\left(i_{l}\right)_{M},\left(j_{k}\right)_{N}}(+1, \pm 1, J) & =e^{ \pm \alpha_{(1,0)}} \prod_{k=1}^{M} \prod_{l=1}^{\cosh \alpha_{\left(1, j_{k}\right)} \times} \\
& \times \cosh \left(\alpha_{\left(1, i_{l}\right)} \mp J\right) \\
\tilde{F}_{\left(i_{l}\right)_{M},\left(j_{k}\right)_{N}}(+1, \pm 1, z, J) & =e^{ \pm \alpha_{(1,0)}} \prod_{k=1}^{N} \prod_{l=1}^{M} \cosh \gamma_{\left(1, j_{k}\right)}(z) \times \\
& \times \cosh \left(\gamma_{\left(1, i_{l}\right)}(z) \mp J\right)
\end{aligned}
$$

onde $\theta=\sum_{i=0}^{n} e_{(1, i)}$ e $\gamma_{(1, i)}(z)=\frac{z e_{(1, i)}+2 \alpha_{(1, i)}}{2}, i=0,1 \ldots, n$

Para simplificar a notação usaremos o símbolo $\mathcal{S}_{\mathcal{M}}$ para indicar a soma binomial sobre listas $\left\{i_{l}\right\}_{l=1}^{M}$ e $\left\{j_{k}\right\}_{k=1}^{N}$ de tomadores do portfólio considerado. Em (2.80), por exemplo, escrevemos $G(z)=\mathcal{S}_{\mathcal{M}} G\left(\left(i_{l}\right)_{M},\left(j_{k}\right)_{N}, \vec{J}\right)$. 
Teorema 2.7 Considere um portfólio de crédito $\Lambda=\{0,1, . ., n\}$ com exposições $\left\{e_{1},,, e_{n}\right\}$ e probabilidades individuais incondicionais de default $\left\{p_{1},,, p_{n}\right\}$ com uma microestrutura de centro produtivo $G(\Lambda, c, J)$ e $L: \mathcal{N} \mapsto \mathbb{R}^{+}$a perda associada ao portfólio $\Lambda$ definida no espaço de probabilidade $(\mathcal{N}, \mathcal{P}(\mathcal{N}), \mathbb{P})$, onde $\mathbb{P}: \mathcal{P}(\mathcal{N}) \mapsto[0,1]$ é definida em (2.25). Então, dado $t \in \mathbb{R}^{+}$, existe $\xi_{t} \in \mathbb{R}$ tal que, para a distribuição de perdas do portfólio $\Lambda$ em torno de $J=0$, a probabilidade cumulativa $\mathbb{P}(L>t)$ resulta em uma função dada pela seguinte expressão:

$$
\mathbb{P}(L>t)=\exp \left(\Theta_{1}\left(\xi_{t}, J\right)-t \xi_{t}+\frac{1}{2} \xi_{t}^{2} \Theta_{2}\left(\xi_{t}, J\right)\right) N\left(-\sqrt{\xi_{t}^{2} \Theta_{2}\left(\xi_{t}, J\right)}\right)
$$

com $N$ sendo a função de distribuição normal acumulativa e

$$
\begin{aligned}
& \Theta_{1}\left(\xi_{t}, J\right)=X^{(0)}\left(\xi_{t}\right)+X^{(1)}\left(\xi_{t}\right) J+\frac{1}{2} X^{(2)}\left(\xi_{t}\right) J^{2} . \\
& \Theta_{2}\left(\xi_{t}, J\right)=Z^{(0)}\left(\xi_{t}\right)+Z^{(1)}\left(\xi_{t}\right) J+\frac{1}{2} Z^{(2)}\left(\xi_{t}\right) J^{2} .
\end{aligned}
$$

onde $X^{(0)}(z), X^{(1)}(z), X^{(2)}(z), Z^{(0)}(z), Z^{(1)}(z)$ e $Z^{(2)}(z)$ são dados em (2.71), (2.72),(2.73),(2.77),(2.78) e (2.79), respectivamente.

Prova: Consideremos inicialmente a função:

$$
\begin{aligned}
K: & \mathbb{R} \times \mathbb{R} \rightarrow \mathbb{R}^{+} \\
(z, J) & \rightarrow K(z, J)=K(z)
\end{aligned}
$$

ou seja, para cada $J \in \mathbb{R}^{+}, K(z, J)$ é a função cumulante definida em (2.65). Dado $t \in \mathbb{R}^{+}$e $J \in \mathbb{R}$, pelo teorema 3.5 temos que existe um único $\xi_{t} \in \mathbb{R}$ tal que:

$$
\left.\mathbb{P}(L>t) \simeq \exp \left(K\left(\xi_{t}, J\right)\right)-t \xi_{t}+\frac{1}{2} \xi_{t}^{2} \frac{d^{2}}{d z^{2}} K\left(\xi_{t}, J\right)\right) N\left(-\sqrt{\xi_{t}^{2} \frac{d^{2}}{d z^{2}} K\left(\xi_{t}, J\right)}\right)
$$


onde $\mathrm{N}$ é a função de distribuição normal acumulativa.

Para mostrar a relação (2.91) calcularemos a expansão de Taylor de ordem 2 em torno do ponto $J=0$ para as funções $K(z, J)$ e $K^{\prime \prime}(z, J)$, ou seja,

$$
K(z, J)=K(z, 0)+D_{J} K(z, 0) J+\frac{1}{2} D_{J J} K(z, 0) J^{2}+o\left(J^{2}\right)
$$

e

$$
K^{\prime \prime}(z, J)=K^{\prime \prime}(z, 0)+D_{J} K^{\prime \prime}(z, 0) J+\frac{1}{2} D_{J J} K^{\prime \prime}(z, 0) J^{2}+o\left(J^{2}\right)
$$

onde adotamos as notações $D_{J} K(z, J)=\frac{\partial}{\partial J} K(z, J)$ e $K^{\prime \prime}(z, J)=\frac{\partial^{2}}{\partial z^{2}} K(z, J)$.

Relambrando, temos por (2.65) que $K(z, J)$ é dado por:

$$
K(z, J)=\log (G(z, J))
$$

$K(z, 0)$ é o cumulante para o caso de eventos independentes. Nesse caso, lembrando que $\left\{i_{l}\right\}_{l=1}^{M}$ e $\left\{j_{k}\right\}_{k=1}^{N}$ são complementares em $\{1, . ., n\}$, obtemos:

$$
F_{\left(i_{l}\right)_{M}}(+1, \pm, 0)=e^{ \pm \alpha_{(1,0)}} \prod_{i=0}^{n} \cosh \alpha_{(1, i)}
$$

e

$$
\tilde{F}_{\left(i_{l}\right)_{M}}(+1, \pm, z, 0)=e^{ \pm \gamma_{(1,0)}(z)} \prod_{i=0}^{n} \cosh \gamma_{(1, i)}(z)
$$

e portanto, usando (2.81),(2.82) e (2.83), segue facilmente que:

$$
G\left(\left(i_{l}\right)_{M},\left(j_{k}\right)_{N}, z, 0\right)=e^{z \theta} \prod_{i=0}^{n} \frac{\cosh \gamma_{(1, i)}(z)}{\cosh \alpha_{(1, i)}}
$$


Assim:

$$
\begin{aligned}
G(z) & =\mathcal{S}_{\mathcal{M}} G\left(\left(i_{l}\right)_{M},\left(j_{k}\right)_{N}, z, 0\right) \\
& =e^{z \theta} \sum_{M=0}^{n} \sum_{\substack{i_{1}<j_{2}<i_{2}<.<j_{n-M} \\
j_{1}}} \prod_{i=0}^{n} \frac{\cosh \gamma_{(1, i)}(z)}{\cosh \alpha_{(1, i)}} c^{M}(1-c)^{n-M} \\
& =e^{z \theta} \prod_{i=0}^{n} \frac{\cosh \gamma_{(1, i)}(z)}{\cosh \alpha_{(1, i)}}(c+(1-c))^{n} \\
& =e^{z \theta} \prod_{i=0}^{n} \frac{\cosh \gamma_{(1, i)}(z)}{\cosh \alpha_{(1, i)}}
\end{aligned}
$$

portanto

$$
\begin{aligned}
K(z, 0) & =\ln G(z, 0) \\
& =z \theta+\sum_{i=0}^{n} \ln \left(\frac{\cosh \gamma_{(1, i)}(z)}{\cosh \alpha_{(1, i)}}\right)
\end{aligned}
$$

Para o cálculo de $D_{J} K(z, 0)$ temos que

$$
D_{J} K(z, 0)=\frac{D_{J} G(z, 0)}{G(z, 0)}
$$

com

$$
\left.D_{J} G(z, J)=e^{z \theta} \mathcal{S}_{\mathcal{M}} D_{J} \tilde{G}\left(\left(i_{l}\right)_{M},\left(j_{k}\right)_{N}\right), z, J\right) .
$$

onde, por (2.82), tem-se que:

$$
\begin{aligned}
D_{J} \tilde{G}\left(\left(i_{l}\right)_{M},\left(j_{k}\right)_{N}, z, J\right) & =\frac{D_{J} H\left(\left(i_{l}\right)_{M},\left(j_{k}\right)_{N}, z, J\right)}{Z\left(\left(i_{l}\right)_{M},\left(j_{k}\right)_{N}, J\right)}- \\
& -\frac{H\left(\left(i_{l}\right)_{M},\left(j_{k}\right)_{N}, z, J\right) D_{J} Z\left(\left(i_{l}\right)_{M},\left(j_{k}\right)_{N}, J\right)}{\left.\left(Z\left(i_{l}\right)_{M},\left(j_{k}\right)_{N}, J\right)\right)^{2}}
\end{aligned}
$$


Portanto, usando que:

$$
\begin{aligned}
Z\left(\left(i_{l}\right)_{M},\left(j_{k}\right)_{N}, 0\right) & =F_{\left(i_{l}\right)_{M},\left(j_{k}\right)_{N}}(+1,+1,0)+ \\
& +F_{\left(i_{l}\right)_{M},\left(j_{k}\right)_{N}}(+1,-1,0) \\
& =2^{n+1} \prod_{i=0}^{n} \cosh \alpha_{(1, i)} \\
H\left(\left(i_{l}\right)_{M},\left(j_{k}\right)_{N}, z, 0\right) & =F_{\left(i_{l}\right)_{M},\left(j_{k}\right)_{N}}(+1,+1,0)+F_{\left(i_{l}\right)_{M},\left(j_{k}\right)_{N}}(+1,-1,0) \\
& =2^{n+1} \prod_{i=0}^{n} \cosh \gamma_{(1, i)}
\end{aligned}
$$

$\mathrm{e}$

$$
G(z, 0)=e^{z \theta} \prod_{1=0}^{n} \frac{\cosh \gamma_{(1, i)}(z)}{\operatorname{senh} \alpha_{(1, i)}} .
$$

segue por $(2.100),(2.101)$ e (2.102), segue que:

$$
D_{J} K(z, 0)=I_{1}^{(0)}(z)-I_{2}^{0}(z)
$$

onde

$$
\begin{aligned}
I_{1}^{(0)}(z) & =\frac{1}{2^{n+1}} \prod_{i=1}^{n} \frac{1}{\cosh \gamma_{(1, i)}(z)} \mathcal{S}_{\mathcal{M}} D_{J} H\left(z,\left(i_{l}\right)_{M},\left(j_{k}\right)_{N}, 0\right) \\
I_{2}^{0}(z) & =\frac{1}{2^{n+1}} \prod_{i=1}^{n} \frac{1}{\cosh \alpha_{(1, i)}(z)} \mathcal{S}_{\mathcal{M}} Z\left(\left(i_{l}\right)_{M},\left(j_{k}\right)_{N}, 0\right)
\end{aligned}
$$

onde, por (2.83) e (2.84), temos:

$$
\begin{aligned}
D_{J} H\left(\left(i_{l}\right)_{M},\left(j_{k}\right)_{N}, z, J\right) & =D_{J} \tilde{F}_{\left(i_{l}\right)_{M},\left(j_{k}\right)_{N}}(+1,+1, z, 0)+ \\
& +D_{J} \tilde{F}_{\left(i_{l}\right)_{M},\left(j_{k}\right)_{N}}(+1,-1, z, 0)
\end{aligned}
$$

e

$$
\begin{aligned}
D_{J} Z\left(J,\left(i_{l}\right)_{M},\left(j_{k}\right)_{N}\right) & =D_{J} F_{\left(i_{l}\right)_{M},\left(j_{k}\right)_{N}}(+1,+1,0)+ \\
& +D_{J} F_{\left(i_{l}\right)_{M},\left(j_{k}\right)_{N}}(+1,-1,0)
\end{aligned}
$$


Por (2.85), segue que:

$$
D_{J} F(+1,+1,0)=-e^{\alpha_{(1,0)}} \sum_{r=1}^{M} \operatorname{senh} \alpha_{\left(1, i_{r}\right)} \prod_{\substack{l \neq 1 \\ l \neq m}}^{M} \prod_{k=1}^{N} \cosh \alpha_{\left(1, i_{l}\right)} \cosh \alpha_{\left(1, i_{k}\right)}
$$

e

$$
D_{J} F(+1,-1,0)=e^{-\alpha_{(1,0)}} \sum_{r=1}^{M} \operatorname{senh} \alpha_{\left(1, i_{r}\right)} \prod_{\substack{l \neq 1 \\ l \neq 1}}^{M} \prod_{k=1}^{N} \cosh \alpha_{\left(1, i_{l}\right)} \cosh \alpha_{\left(1, i_{k}\right)}
$$

portanto,

$$
\begin{aligned}
D_{J} Z\left(\left(i_{l}\right)_{M},\left(j_{k}\right)_{N}, 0\right) & =2^{n+1} \operatorname{senh} \alpha_{(1,0)} \sum_{r=1}^{M} \operatorname{senh} \alpha_{\left(1, i_{r}\right)} \times \\
& \times \prod_{\substack{l \neq 1 \\
l \neq k}}^{M} \prod_{k=1}^{N} \cosh \alpha_{\left(1, i_{l}\right)} \cosh \alpha_{\left(1, i_{k}\right)}
\end{aligned}
$$

Análogamente também temos,

$$
\begin{aligned}
D_{J} H\left(\left(i_{l}\right)_{M},\left(j_{k}\right)_{N}, z, 0\right) & =2^{n+1} \operatorname{senh} \gamma_{(1,0)}(z) \sum_{r=1}^{M} \operatorname{senh} \gamma_{\left(1, i_{r}\right)(z)} \times \\
& \times \prod_{\substack{l \neq 1 \\
l \neq r}}^{M} \prod_{k=1}^{N} \cosh \gamma_{\left(1, i_{l}\right)}(z) \cosh \gamma_{\left(1, i_{k}\right)}(z)
\end{aligned}
$$

Voltando a (2.107) e (2.108), devemos agora calcular as médias:

$$
\mathcal{S}_{\mathcal{M}} D_{J} Z\left(\left(i_{l}\right)_{M},\left(j_{k}\right)_{N}, 0\right)=\sum_{M=0}^{n} \sum_{\substack{i_{1}<i_{2}<\ldots<i_{M} \\ j_{1}<j_{2}<. .<j_{n-M}}} D_{J} Z\left(\left(i_{l}\right)_{M},\left(j_{k}\right)_{N}, 0\right) c^{M}(1-c)^{n-M}
$$

e

$$
\mathcal{S}_{\mathcal{M}} D_{J} H\left(\left(i_{l}\right)_{M},\left(j_{k}\right)_{N}, z, 0\right)=\sum_{M=0}^{n} \sum_{\substack{i_{1}<i_{2}<. .<i_{M} \\ j_{1}<j_{2}<.<j_{n-M}}} D_{J} H\left(\left(i_{l}\right)_{M},\left(j_{k}\right)_{N}, z, 0\right) c^{M}(1-c)^{n-M}
$$


Observe que o produtório duplo em (2.113) é sempre igual ao produto de $n-1$ termos sobre o conjunto de índices $I=\{(1, i) ; i=1,,,, n\}$. Portanto basta observarmos que, para $M=k, k \geq 1$, e $r \in\{1,,, n\}$, o termo

$$
\operatorname{senh} \alpha_{(1, r)} \prod_{\substack{i \neq 1 \\ i \neq j}}^{n} \cosh \alpha_{(1, i)} c^{k}(1-c)^{n-k}
$$

aparece $\left(\begin{array}{c}n-1 \\ k-1\end{array}\right)$ vezes no somatório em (2.114). Portanto temos

$$
\begin{aligned}
\mathcal{S}_{\mathcal{M}} D_{J} Z\left(\left(i_{l}\right)_{M},\left(j_{k}\right)_{N}, 0\right) & =2^{n+1} \sum_{r=1}^{n-1} \sum_{k=1}^{n}\left(\begin{array}{c}
n-1 \\
k-1
\end{array}\right) \operatorname{senh} \alpha_{(1, r)} \times \\
& \times \prod_{\substack{i \neq 1 \\
i \neq r}}^{n} \cosh \alpha_{(1, i)} c^{k}(1-c)^{n-k} \\
& =2^{n+1} c \sum_{j=1}^{n} \operatorname{senh} \alpha_{(1, r)} \prod_{\substack{i \neq 1 \\
i \neq r}}^{n} \cosh \alpha_{(1, i)}(c+(1-c))^{n-1} \\
& =2^{n+1} c \sum_{r=1}^{n} \operatorname{senh} \alpha_{(1, r)} \prod_{\substack{i \neq 1 \\
i \neq r}}^{n} \cosh \alpha_{(1, i)}
\end{aligned}
$$

Análogamente temos:

$$
\begin{aligned}
\mathcal{S}_{\mathcal{M}} D_{J} H\left(\left(i_{l}\right)_{M},\left(j_{k}\right)_{N}, z, 0\right) & =2^{n+1} c \sum_{r=1}^{n} \operatorname{senh} \gamma_{(1, r)}(z) \times \\
& \times \prod_{\substack{i=1 \\
i \neq r}}^{n} \cosh \gamma_{(1, i)}(z)
\end{aligned}
$$

Portanto substituindo (2.118) e (2.117) em (2.107) e (2.108),respectivamente, segue de (2.106)que:

$$
D_{J} K(z, 0)=c \sum_{r=1}^{n}\left(\operatorname{tgh} \gamma_{(1,0)}(z) \operatorname{tgh} \gamma_{(1, r)}(z)-\operatorname{tgh} \alpha_{(1,0)} \operatorname{tgh} \alpha_{(1, r)}\right) .
$$

Para o cálculo de $D_{J J} K(z, 0)$, segue de (2.94) que,

$$
D_{J J} K(z, 0)=I_{1}^{(1)}(z)-I_{2}^{(1)}(z)
$$


onde

$$
I_{1}^{(1)}(z)=\frac{D_{J} J G(z, 0)}{G(z, 0)}
$$

e

$$
I_{1}^{(2)}(z)=\left(\frac{D_{J} J G(z, 0)}{G(z, 0)}\right)^{2}=\left(D_{J} K(z, 0)\right)^{2}
$$

onde $D_{J} K(z, 0)$ é dado em (2.119).

Por (2.81) e (2.82) segue que:

$$
\begin{aligned}
I_{1}^{(1)}(z) & =\frac{e^{z \theta}}{G(z, 0)} \mathcal{S}_{\mathcal{M}} D_{J J} \tilde{G}\left(z, i_{l}, j_{k}, 0\right)= \\
& =\frac{e^{z \theta}}{G(z, 0)}\left(\mathcal{S}_{\mathcal{M}} D_{J} P_{1}(z, 0)-\mathcal{S}_{\mathcal{M}} D_{J} P_{2}(z, 0)\right)
\end{aligned}
$$

onde

$$
P_{1}(z, J)=\frac{D_{J} H\left(\left(i_{l}\right)_{M},\left(j_{k}\right)_{N}, z, 0\right)}{Z\left(\left(i_{l}\right)_{M},\left(j_{k}\right)_{N}, 0\right)}
$$

e

$$
P_{2}(z, J)=\frac{H\left(\left(i_{l}\right)_{M},\left(j_{k}\right)_{N}, z, 0\right) D_{J} Z\left(\left(i_{l}\right)_{M},\left(j_{k}\right)_{N}, 0\right)}{\left(Z\left(i_{l}, j_{k}, 0\right)\right)^{2}}
$$

Portanto temos que:

$$
I_{1}^{(1)}(z)=I_{1,1}^{(1)}+I_{1,2}^{(1)}(z)
$$

onde

$$
I_{1,1}^{(1)}(z)=\frac{e^{z \theta}}{G(z, 0)} \mathcal{S}_{\mathcal{M}} D_{J} P_{1}(z, 0)
$$

e

$$
I_{1,2}^{(1)}(z)=\frac{e^{z \theta}}{G(z, 0)} \mathcal{S}_{\mathcal{M}} D_{J} P_{2}(z, 0)
$$


Usando que (2.104),(2.104),(2.105) e (2.124), segue que:

$$
\begin{aligned}
I_{1,1}^{(1)}(z) & =\frac{e^{z \theta}}{G(z, 0)}\left\{\frac{1}{2^{n+1}} \prod_{i=0}^{n} \frac{1}{\cosh \alpha_{(1, i)}} \mathcal{S}_{\mathcal{M}} D_{J J} H\left(i_{l}, j_{k}, z, 0\right)-\right. \\
& -\left(\frac{1}{2^{n+1}} \prod_{i=0}^{n} \frac{1}{\cosh \alpha_{(1, i)}}\right)^{2} \times \\
& \left.\times \mathcal{S}_{\mathcal{M}}\left(D_{J} H\left(\left(i_{l}\right)_{M},\left(j_{k}\right)_{N}, z, 0\right) D_{J} Z\left(\left(i_{l}\right)_{M},\left(j_{k}\right)_{N}, 0\right)\right)\right\}= \\
& =I_{1,1,1}^{(1)}(z)-I_{1,1,2}^{(1)}(z)
\end{aligned}
$$

onde

$$
\begin{aligned}
I_{1,1,1}^{(1)}(z) & =\frac{1}{2^{n+1}} \prod_{i=0}^{n} \frac{1}{\cosh \alpha_{(1, i)}} \mathcal{S}_{\mathcal{M}} D_{J J} H\left(\left(i_{l}\right)_{M},\left(j_{k}\right)_{N}, z, 0\right) \\
I_{1,1,2}^{(2)}(z) & =\left(\frac{1}{2^{n+1}} \prod_{i=0}^{n} \frac{1}{\cosh \alpha_{(1, i)} \cosh \gamma_{(1, i)}(z)}\right) \times \\
& \times \mathcal{S}_{\mathcal{M}} D_{J}\left(H\left(\left(i_{l}\right)_{M},\left(j_{k}\right)_{N}, z, 0\right) D_{J} Z\left(\left(i_{l}\right)_{M},\left(j_{k}\right)_{N}, 0\right)\right)
\end{aligned}
$$

Para o cálculo de $\mathcal{S}_{\mathcal{M}} D_{J}\left(H\left(\left(i_{l}\right)_{M},\left(j_{k}\right)_{N}, z, 0\right) D_{J} Z\left(\left(i_{l}\right)_{M},\left(j_{k}\right)_{N}, 0\right)\right)$, relembremos de $(2.113)$ e (2.114) que:

$$
\begin{aligned}
D_{J} Z\left(\left(i_{l}\right)_{M},\left(j_{k}\right)_{N}, 0\right) & =2^{n+1} \operatorname{senh} \alpha_{(1,0)} \sum_{r=1}^{M} \operatorname{senh} \alpha_{\left(1, i_{r}\right)} \times \\
& \times \prod_{\substack{l=1 \\
l \neq k}}^{M} \prod_{k=1}^{N} \cosh \alpha_{\left(1, i_{l}\right)} \cosh \alpha_{\left(1, i_{k}\right)}
\end{aligned}
$$

e

$$
\begin{aligned}
D_{J} H\left(\left(i_{l}\right)_{M},\left(j_{k}\right)_{N}, z, 0\right) & =2^{n+1} \operatorname{senh} \gamma_{(1,0)}(z) \sum_{r=1}^{M} \operatorname{senh} \gamma_{\left(1, i_{r}\right)(z)} \times \\
& \times \prod_{\substack{l \neq 1 \\
l \neq r}}^{M} \prod_{k=1}^{N} \cosh \gamma_{\left(1, i_{l}\right)}(z) \cosh \gamma_{\left(1, i_{k}\right)}(z)
\end{aligned}
$$


Observe que, para cada $i, j \in\{1, n\}$ temos que o termo:

$$
\operatorname{senh} \gamma_{(1, i)}(z, 0) \operatorname{senh} \alpha_{(1, j)} \prod_{\substack{l=1 \\ l \neq i}}^{n} \prod_{\substack{t \neq 1 \\ t \neq j}}^{n} \cosh \alpha_{(1, l)} \cosh \gamma_{(1, t)} c^{k}(1-c)^{n-k}
$$

aparece $\left(\begin{array}{c}n-2 \\ k-2\end{array}\right)$ no somatório $\mathcal{S}_{\mathcal{M}} D_{J}\left(H\left(\left(i_{l}\right)_{M},\left(j_{k}\right)_{N}, z, 0\right) D_{J} Z\left(\left(i_{l}\right)_{M},\left(j_{k}\right)_{N}, 0\right)\right)$ para $M=k \geq 2$ e portanto:

$$
\begin{aligned}
\left.\mathcal{S}_{\mathcal{M}} D_{J} H\left(\left(i_{l}\right)_{M},\left(j_{k}\right)_{N}, z, 0\right) D_{J} Z\left(\left(i_{l}\right)_{M},\left(j_{k}\right)_{N}, 0\right)\right) & =2^{(n+1)} \operatorname{senh} \alpha_{(1,0)} \times \\
& \times \operatorname{senh} \gamma_{(1,0)}(z, 0) U(z)
\end{aligned}
$$

onde

$$
\begin{aligned}
U(z) & \left.=\sum_{i=1}^{n} \sum_{j=1}^{n} \operatorname{senh} \alpha_{(1, i)} \operatorname{senh} \gamma_{(1, j)}(z, 0) \prod_{\substack{l=1 \\
l \neq i}}^{n} \prod_{\substack{t=1 \\
t \neq j}}^{n} \cosh \alpha_{(1, l)} \cosh \gamma_{(1, t)}\right) \times \\
& \times \sum_{k=2}^{n}\left(\begin{array}{c}
n-2 \\
k-2
\end{array}\right) c^{k}(1-c)^{n-k}
\end{aligned}
$$

onde

$$
\sum_{k=2}^{n}\left(\begin{array}{l}
n-2 \\
k-2
\end{array}\right) c^{k}(1-c)^{n-k}=c^{2}(c+(1-c))^{n-2}=c^{2}
$$

Portanto, segue de (2.131) que:

$$
I_{1,1,2}^{(1)}(z)=c^{2} \operatorname{tgh} \alpha_{(1,0)} \operatorname{tgh} \gamma_{(1,0)} \sum_{i=1}^{n} \sum_{j=1}^{n} \operatorname{tgh} \alpha_{(1, i)} \operatorname{tgh} \gamma_{(1, i)}(z) .
$$

Para o cálculo de $I_{1,1,1}^{1}(z)$, de 2.130 temos que:

$$
I_{1,1,1}^{(1)}(z)=\frac{1}{2^{n+1}} \prod_{i=0}^{n} \frac{1}{\cosh \alpha_{(1, i)}} \mathcal{S}_{\mathcal{M}} D_{J J} H\left(\left(i_{l}\right)_{M},\left(j_{k}\right)_{N}, z, 0\right) .
$$


Por (2.83) temos que

$$
\begin{aligned}
D_{J J} H\left(\left(i_{l}\right)_{M},\left(j_{k}\right)_{N}, z, J\right) & =D_{J J} \tilde{F}_{\left(i_{l}\right)_{M},\left(j_{k}\right)_{N}}(+1,+1, z, J)+ \\
& +D_{J J} \tilde{F}_{\left(i_{l}\right)_{M},\left(j_{k}\right)_{N}}(+1,-1, z, J)
\end{aligned}
$$

onde por (2.85) e (2.85), obtemos:

$$
D_{J J} \tilde{F}_{\left(i_{l}\right)_{M},\left(j_{k}\right)_{N}}(+1, \pm 1, z, 0)=e^{\mp \gamma_{(1,0)}(z)} \sum_{r=1}^{M}\left(Q_{r}(z)+\operatorname{senh} \gamma_{(1, r)}(z) T_{r, s}(z)\right)
$$

onde

$$
Q_{r}(z)=\cosh \gamma_{\left(1, j_{r}\right)}(z) \prod_{\substack{l \neq 1 \\ l \neq r}}^{M} \prod_{k=1}^{N} \cosh \gamma_{\left(1, i_{l}\right)}(z) \cosh \gamma_{\left(1, j_{k}\right)}
$$

e

$$
\begin{aligned}
T_{r, s}(z) & =\operatorname{senh} \gamma_{\left(1, j_{r}\right)}(z) \sum_{\substack{s=1 \\
s \neq r}} \operatorname{senh} \gamma_{\left(1, j_{r}\right)}(z) \times \\
& \times \prod_{\substack{l=1 \\
l \neq 1, s}}^{M} \prod_{k=1}^{N} \cosh \gamma_{\left(1, i_{l}\right)}(z) \cosh \gamma_{\left(1, j_{k}\right)}
\end{aligned}
$$

portanto, por (2.141), segue que:

$$
\begin{aligned}
D_{J J} H\left(\left(i_{l}\right)_{M},\left(j_{k}\right)_{N}, z, 0\right) & =2^{n+1} \cosh \gamma_{(1,0)}(z) \times \\
& \times \sum_{r=1}^{M}\left(Q_{r}(z)+\operatorname{senh} \gamma_{(1, r)}(z) T_{r, s}(z)\right)
\end{aligned}
$$

Pelo mesmo processo de contagem feito em casos anteriores obtemos:

$$
\begin{aligned}
\mathcal{S}_{\mathcal{M}} D_{J J} H\left(\left(i_{l}\right)_{M},\left(j_{k}\right)_{N}, z, 0\right) & =2^{n+1} \cosh \gamma_{(1,0)}(z) \times \\
& \times \sum_{r=1}^{n}\left(c \cdot \tilde{Q}_{r}(z)+c \cdot \operatorname{senh} \gamma_{(1, r)}(z) \tilde{T}_{r, s}(z)\right)
\end{aligned}
$$


onde

$$
\tilde{Q}_{r}(z)=\cosh \gamma_{(1, r)}(z) \prod_{\substack{l \neq 1 \\ l \neq r}}^{n} \cosh \gamma_{(1, l)}(z)
$$

e

$$
\tilde{T}_{r, s}(z)=\operatorname{senh} \gamma_{(1, r)}(z) \sum_{\substack{s=1 \\ s \neq r}}^{n} \operatorname{senh} \gamma_{(1, r)}(z) \prod_{\substack{l=1 \\ l \neq r, s}}^{n} \cosh \gamma_{(1, l)}(z)
$$

Voltando a (2.140), finalmente, usando (2.148) obtemos:

$$
I_{1,1,1}^{(1)}(z)=\sum_{r=1}^{n} c\left\{1+c \cdot \operatorname{tgh} \gamma_{(1, r)}(z) \sum_{\substack{s \neq 1 \\ s \neq r}} \operatorname{tgh} \gamma_{((1, s))}(z)\right\}
$$

Voltando a (2.129), concluímos então que:

$$
I_{1,1}^{(1)}(z)=I_{1,1,1}^{(1)}(z)-I_{1,1,2}^{(1)}(z)
$$

onde $I_{1,1,1}^{(1)}(z)$ e $I_{1,1,2}^{(1)}(z)$ são dados em (2.151) e (2.139), respectivamente.

Por último precisamos calcular $I_{1,2}^{(1)}$ em (2.128), que é dado por:

$$
I_{1,2}^{(1)}(z)=\frac{e^{z \theta}}{G(z, 0)} \mathcal{S}_{\mathcal{M}} D_{J} P_{2}(z, 0)
$$

onde $P_{2}(z, J)$ é definido em (2.125).

Derivando $P_{2}(z, J)$ em (2.125) obtemos facilmente que:

$$
\begin{aligned}
I_{1,2}^{(1)}(z) & =\frac{D_{J} H\left(z, i_{l}, j_{k}, 0\right) D_{J} z\left(i_{l}, j_{k}, 0\right)}{\left(z\left(i_{l}, j_{k}, 0\right)\right)^{2}}+ \\
& +\frac{H\left(z, i_{l}, j_{k}, 0\right)}{\left(Z\left(i_{l}, j_{k}, 0\right)\right)^{2}} \cdot D_{J J} Z\left(i_{l}, j_{k}, 0\right)+ \\
& -2 \frac{H\left(z, i_{l}, j_{k}, 0\right)}{\left(Z\left(i_{l}, j_{k}, 0\right)\right)^{3}} \cdot\left(D_{J} Z\left(i_{l}, j_{k}, 0\right)\right)^{2} .
\end{aligned}
$$

Portanto usando (2.104) e (2.104), segue de (2.153), que:

$$
\mathcal{S}_{\mathcal{M}} D_{J} P_{2}(z, J)=I_{1,2,1}^{(1)}(z)+I_{1,2,2}^{(1)}(z)-I_{1,2,2}^{(1)}(z)
$$


onde,

$$
\begin{aligned}
I_{1,2,1}^{(1)}= & \left(\frac{1}{2^{2(n+1)}} \prod_{i=0}^{n} \frac{1}{\cosh \gamma_{(1, i)}(z) \cosh \alpha_{(1, i)}}\right) \times \\
\times & \mathcal{S}_{\mathcal{M}} D_{J}\left(H\left(\left(i_{l}\right)_{M},\left(j_{k}\right)_{N}, z, 0\right) D_{J} Z\left(\left(i_{l}\right)_{M},\left(j_{k}\right)_{N}, 0\right)\right) \\
= & I_{1,1,2}^{(1)}(z) \\
I_{1,2,2}^{(1)}(z) & =\left(\frac{1}{2^{(n+1)}} \prod_{i=0}^{n} \frac{1}{\cosh \alpha_{(1, i)}}\right) \cdot \mathcal{S}_{\mathcal{M}} D_{J J} Z\left(i_{l}, j_{k}, 0\right) . \\
& =\sum_{r=1}^{n} c\left\{1+\operatorname{c.tgh} \alpha_{(1, r)} \sum_{\substack{s=1 \\
s \neq r}} \operatorname{tgh} \alpha_{((1, s))}\right\}
\end{aligned}
$$

e

$$
\begin{aligned}
I_{1,2,3}^{(1)}(z) & =2\left(\frac{1}{2^{(n+1)}} \prod_{i=0}^{n} \frac{1}{\cosh \alpha_{(1, i)}}\right) \mathcal{S}_{\mathcal{M}}\left(D_{J} Z\left(i_{l}, j_{k}, 0\right)\right)^{2} \\
& =2\left(\operatorname{c.tgh} \alpha_{(1,0)} \sum_{r=1}^{n} \operatorname{tgh} \alpha_{(1, r)}\right)
\end{aligned}
$$

onde $(2.158)$ e (2.158) são obtidos de $(2.117)$ e $(2.148)$ observando que $Z\left(i_{l}, j_{k}, 0\right)$ e $H\left(z, i_{l}, j_{k}, 0\right)$ são expressões análogas.

Voltando a (2.99),(2.119) e (2.120), definindo :

$$
\begin{aligned}
& X^{(0)}\left(\xi_{t}\right)=K\left(\xi_{t}, 0\right) \\
& X^{(1)}\left(\xi_{t}\right)=D_{J} K\left(\xi_{t}, 0\right)
\end{aligned}
$$

$\mathrm{e}$

$$
X^{(2)}\left(\xi_{t}\right)=D_{J J} K\left(\xi_{t}, 0\right)
$$

e obtemos $\Theta_{1}\left(\xi_{t}, J\right)$ em (2.88).

Os outros coeficientes, $K^{\prime \prime}(z, 0) D_{J} K^{\prime \prime}(z, 0)$ e $D_{J J} K^{\prime \prime}(z, 0)$ são obtidos simplesmente derivando as somas $k(z, 0), D_{J} K(z, 0)$ e $D_{J J} K(z, 0)$ na variável z e não 
faremos aqui. Os valores são dados por $Z^{(0)}(z), Z^{(1)}(z)$ e $Z^{(2)}(z)$, em $(2.77),(2.78)$ e (2.79), respectivamente, resultando (fazendo $z=\xi_{t}$ ) nos coeficientes de $\Theta_{2}\left(\xi_{t}\right)$ em (2.89).

Observação 4 Observe que se supomos que a probabilidade de existência de interação entre os tomadores do portfólio seja sempre zero, ou seja, c $=0$, então as funções $X^{1}(z), X^{2}(z), Z^{1}(z), Z^{2}(z)$ dadas em (2.72),(2.73),(2.78) e (2.79), respectivamente, se anulam e portanto os coeficientes $D_{J} K(z, 0), D_{J J} K(z, 0), D_{J} K^{\prime \prime}(z, 0)$ e $D_{J J} K^{\prime}(z, 0)$ também se anulam em (2.92) e (2.93) reduzindo o cumulante ao caso independente, como naturalmente deveria ocorrer.

Observação 5 O teorema 3.6 de fato nos fornece uma expressão analítica bastante razoável para estimar a cauda da distribuição de perdas do portfólio haja vista que os valores encontrados para $\Theta_{1}\left(\xi_{t}, J\right)$ e $\Theta_{2}\left(\xi_{t}, J\right)$ são polinômios cujos coeficientes se reduzem a somas de ordem $O(n), O\left(n^{2}\right)$ ou $O\left(n^{3}\right)$. Observe ainda que, em vizinhanças próximas de $J=0$, como sugere o teorema anterior, o ponto de sela, o qual depende a aproximação da cauda da distribuição em (2.91), pode ser considerado como solução da equação:

$$
\left.\frac{d}{d z} K(z, J)\right|_{z=\xi_{t}}=t
$$

onde

$$
K^{\prime}(z, J)=K^{\prime}(z, 0)+D_{J} K^{\prime}(z, 0) J+\frac{1}{2} D_{J J} K^{\prime}(z, 0) J^{2}+o\left(J^{2}\right) .
$$

cujos coeficientes também se reduzem a uma complexidade de ordem $O(n), O\left(n^{2}\right)$, ou $O\left(n^{3}\right)$, facilitando a sua determinação por um processo simples de interpolação linear. $K^{\prime}(z, 0) D_{J} K^{\prime}(z, 0)$ e $D_{J J} K^{\prime}(z, 0)$ são dados por (2.74),(2.75)e (2.76), respectivamente . 


\subsubsection{Conclusões e Estudos Futuros}

Conforme mostramos neste trabalho, claramente obtemos um método extremamente eficiente para estimar a distribuição de perdas em portfólios de crédito onde os tomadores interagem microeconômicamente entre si num contexto de centro produtivo. Naturalmente essa eficiência se traduz em expressões aproximadas que são facilmente implementáveis e que foram obtidas como resultado da metodologia apresentada. Um ponto relevante que sem dúvida alguma valoriza este trabalho se deve as possibilidades de extensões de seus resultados que podem ser consideradas em diversas direções. Uma primeiro caminho, certamente viável, seria aplicar as técnicas apresentadas para estudar a influência de contágio econômico sobre o default de um tomador em particular. Observe que as probabilidades individuais de default determinadas pela proposição 2.3 se expressam de maneira análoga as expressões da função geradora da perda definida em 2.59 e portanto, no contexto de contágio uniforme em torno de zero, certamente podemos também eliminar sua complexidade exponencial obtendo novamente uma estimativa mais fácil de ser implementada.

Uma segunda abordagem interessante para as técnicas desenvolvidas neste trabalho seria a sua utilização para o cálculo da decomposição do risco total, definido como a variância da perda, onde se separa a parcela do risco responsável pelo contágio econômico possibilitando uma análise mais eficiente sobre o efeito de fatores microeconômicos sobre a distribuição de perdas do portfólio. Podemos fazer isso utilizando a fórmula da variância condicional sobre a perda L do 
portfólio de perdas ${ }^{2}$, resultando na seguinte decomposição:

$$
\sigma^{2}(L)=E_{G(n, c)}\left[\sigma_{L}^{2}[L \mid J(g)]\right]+\sigma_{G(n, c)}^{2}[E[L \mid J(g)]]
$$

onde $E_{G(n, c)}$ e $\sigma_{G(n, c)}^{2}$ são, respectivamente, a esperança e a variância calculada sobre a classe de microestruturas de centros produtivos $G(\Lambda, c, J)$. A segunda expressão em (2.165) é a parcela responsável pelo contágio pois representa a variância de uma função da variável $\mathrm{J}(\mathrm{g})$. A variância na primeira parcela é calculada conhecendo-se a topologia de contágio e a sua esperança representa então o componente macroeconômico do risco total.

Utilizando cálculos alementares podemos desenvolver as duas parcelas em (2.165) obtendo:

$$
\begin{aligned}
E\left[\sigma^{2}[L \mid J(g)]\right] & =\sum_{M=0}^{n} \sum_{i_{1}<i_{2}<. .<i_{M}}\left\{\sum_{l=1}^{M} e_{\left(1, i_{l}\right)}^{2} \mathbb{P}_{\left(1, i_{l}\right)}\left(1-\mathbb{P}_{\left(1, i_{l}\right)}\right)+\right. \\
& \left.+\sum_{k, l=1}^{M} e_{\left(1, i_{k}\right)} e_{\left(1, i_{l}\right)}\left(\mathbb{P}_{k, l}^{*}-\mathbb{P}_{\left(1, i_{k}\right)} \mathbb{P}_{\left(1, i_{l}\right)}\right)\right\} c^{M}(1-c)^{n_{1}-M}
\end{aligned}
$$

e

$$
\begin{aligned}
\sigma^{2}[E[L \mid J(g)]] & =\sum_{M=0}^{n} \sum_{i_{1}<i_{2}<. .<i_{M}}\left\{\sum_{k, l=1}^{M} e_{\left(1, i_{k}\right)} e_{\left(1, i_{l}\right)} \mathbb{P}_{\left(1, i_{k}\right)} \mathbb{P}_{\left(1, i_{l}\right)}\right\} c^{M}(1-c)^{n_{1}-M}- \\
& \left.\left.-\left(\sum_{M=0}^{n_{1}} \sum_{i_{1}<i_{2}<. .<i_{M}} \sum_{l=1}^{M} e_{\left(1, i_{l}\right)} \mathbb{P}_{\left(1, i_{l}\right)}\right\} c^{M}(1-c)^{n_{1}-M}\right)^{2}\right\}
\end{aligned}
$$

onde

$$
\mathbb{P}_{(1,0)}=2^{M} \frac{e^{\alpha_{(1,0)}}}{Z(g)} \prod_{i=l}^{M} \cosh \left(\alpha_{\left(1, i_{l}\right)}-J\right),
$$

\footnotetext{
${ }^{2}$ veja [Ross,2002], pag. 355
} 


$$
\begin{aligned}
\mathbb{P}_{\left(1, i_{j}\right)} & =2^{M-1} \frac{e^{\alpha_{\left(1, i_{j}\right)}}}{Z(g)}\left(e^{\left(\alpha_{(1,0)}-J\right)} \prod_{\substack{i \neq 1 \\
l \neq j}}^{M} \cosh \left(\alpha_{\left(1, i_{l}\right)}-J\right)+\right. \\
& \left.+e^{\left(-\alpha_{(1,0)}+J\right)} \prod_{\substack{i=1 \\
l \neq j}}^{n_{1}} \cosh \left(\alpha_{\left(1, i_{l}\right)}+J\right)\right)
\end{aligned}
$$

$\mathrm{e}$

$$
\begin{aligned}
\mathbb{P}_{k, l}^{*} & =\frac{2^{n-3} e^{\alpha_{\left(1, i_{l}\right)}+\alpha_{\left(1, i_{k}\right)}}}{Z(g)} \times \\
& \times\left(e^{\alpha_{(1,0)}-2 J} \cdot \prod_{\substack{s=1 \\
s \neq k, l}}^{M} \cosh \left(\alpha_{\left(1, i_{l}\right)}-J\right)+e^{-\alpha_{(1,0)}+2 J} \prod_{\substack{s=1 \\
s \neq k, l}}^{M} \cosh \left(\alpha_{\left(1, i_{l}\right)}+J\right)\right)
\end{aligned}
$$

com

$$
Z(g)=2^{M}\left(e^{\alpha_{(1,0)}} \prod_{l=1}^{M} \cosh \left(\alpha_{\left(1, i_{l}\right)}-J\right)+e^{-\alpha_{(1,0)}} \prod_{l=1}^{M} \cosh \left(\alpha_{\left(1, i_{l}\right)}+J\right)\right),
$$

para $1 \leq j, k, l \leq M$

Novamente a partir deste ponto a expansão de taylor em torno de $J=0$ para as duas parcelas da decomposição obviamente se mostra viável para a análise das mesmas.

Finalmente o terceiro caminho natural para o prosseguimento deste trabalho seria a sua extensão para outras topologias de contágio . Os procedimentos utilizados no contexto de centro produtivo podem, sem dúvida alguma, ser repetidos para outros modelos conforme descrevemos no início deste capítulo. Como exemplo consideremos um caso particular de um cadeia produtiva com dois clusters dependentes, como mostra a figura abaixo, 




Neste caso, temos que a função geradora para um grafo g sorteado aleatoriamente é expressa como:

$$
\begin{aligned}
G_{g}(z)= & \frac{e^{z \theta} 2^{n+m-2}}{Z(g)} \prod_{p=1}^{r} \prod_{q=1}^{s} \cosh \gamma_{\left(1, i_{p}\right)}(z) \cosh \gamma_{\left(1, j_{q}\right)}(z) \times \\
\times & {\left[e^{\gamma_{(1,0)}(z)+\gamma_{(2,0)}(z)-\epsilon} \prod_{k=1}^{m-r} \cosh \left(\gamma_{\left(1, i_{k}\right)}(z)-J_{1}\right) \prod_{l=1}^{n-s} \cosh \left(\gamma_{\left(1, j_{l}\right)}(z)-J_{2}\right)\right.} \\
& +e^{\gamma_{(1,0)}(z)-\gamma_{(2,0)}(z)+\epsilon} \prod_{k=1}^{m-r} \cosh \left(\gamma_{\left(1, i_{k}\right)}(z)-J_{1}\right) \prod_{l=1}^{n-s} \cosh \left(\gamma_{\left(1, j_{l}\right)}(z)+J_{2}\right) \\
& +e^{-\gamma_{(1,0)}(z)+\gamma_{(2,0)}(z)+\epsilon} \prod_{k=1}^{m-r} \cosh \left(\gamma_{\left(1, i_{k}\right)}(z)+J_{1}\right) \prod_{l=1}^{n-s} \cosh \left(\gamma_{\left(1, j_{l}\right)}(z)-J_{2}\right) \\
& \left.+e^{-\gamma_{(1,0)}(z)-\gamma_{(2,0)}(z)-\epsilon} \prod_{k=1}^{m-r} \cosh \left(\gamma_{\left(1, i_{k}\right)}(z)+J_{1}\right) \prod_{l=1}^{n-s} \cosh \left(\gamma_{\left(1, j_{l}\right)}(z)+J_{2}\right)\right]
\end{aligned}
$$


onde

$$
\begin{aligned}
& Z(g)=\left[e^{\alpha_{(1,0)}+\alpha_{(2,0)}-\epsilon} \prod_{k=1}^{m-r} \cosh \left(\alpha_{\left(1, i_{k}\right)}-J_{1}\right) \prod_{l=1}^{n-s} \cosh \left(\alpha_{\left(1, j_{l}\right)}-J_{2}\right)\right. \\
& +e^{\alpha_{(1,0)}-\alpha_{(2,0)}+\epsilon} \prod_{k=1}^{m-r} \cosh \left(\alpha_{\left(1, i_{k}\right)}-J_{1}\right) \prod_{l=1}^{n-s} \cosh \left(\alpha_{\left(1, j_{l}\right)}+J_{2}\right) \\
& \left.+e^{-\alpha_{(1,0)}+\alpha_{(2,0)}+\epsilon} \prod_{k=1}^{m-r} \cosh \left(\alpha_{\left(1, i_{k}\right)}\right)+J_{1}\right) \prod_{l=1}^{n-s} \cosh \left(\alpha_{\left(1, j_{l}\right)}-J_{2}\right) \\
& \left.+e^{-\alpha_{(1,0)}-\alpha_{(2,0)}-\epsilon} \prod_{k=1}^{m-r} \cosh \left(\alpha_{\left(1, i_{k}\right)}+J_{1}\right) \prod_{l=1}^{n-s} \cosh \left(\alpha_{\left(1, j_{l}\right)}+J_{2}\right)\right]
\end{aligned}
$$

com $\gamma_{(1, i)}=\frac{e_{(1, i)}+2 \alpha_{(1, i)}}{2}$ para todo $i$.

Portanto, claramente, todos os procedimentos utilizados nos casos anteriores podem ser usados aqui de maneira similar. Acreditamos que outras estruturas básicas podem também ser investigadas com sucesso, pois apresentam o mesmo padrão de complexidade analítica.

Com isso podemos concluir que o modelo apresentado representa de fato um conjunto de ferramentas promissoras que certamente podem ser ainda mais desenvolvidas com o intuito de analizar portfólios de créditos sujeitos a cenários microeconômicos que são frequentemente observados no mundo econômico. 


\section{Referências Bibliográficas}

[Bielecki,2002] BIELECKI,T.R., E RUTKOWSKI, M., Credit Risk: Modeling, Valuation and Hedging. $1_{a}$ edição.Berlin, Springer-Verlag, 2002.

[Black,1973] BLACK,F. e SCHOLES,M. The pricing of options and corporate liabilities. J. Political Economy, 81:637-654, 1973.

[Black,1976] BLACK,F. e COX, J.C., Valuing corporate securities: Some effects of bond indenture provisions. J. Finance, 31:351-367, 1976.

[Bollobás,1998] BOLLOBÁS BÉLA, Modern graph theory, Graduate Texts in Mathematics, Springer Verlag, 1998.

[Christian,2000] BLUHN C., OVERBECK L. E WAGNER C., An Introduction to Credit Risk Modeling, Chapman \& Hall/CRC, Financial Mathematical Series, 2000.

[Credit Suisse,1997] CREDIT SUISSE, CreditRisk+: A Credit Risk Management Framework Credit Suisse Financial Products,1997.

[Brys,1997] BRYS, E. DE VERENNE, F., Valuing risk fixed rate debt: An extension. J. Financial end Quant. Anal., 32: 239-248,1997.

[Duffie, 1999] DUFFIE, D. e SINGLETON, K. Modeling term structures of defaultable bonds. Rev. Finan. Stud., 12:687-720,1999. 
[Duffie, 2003] DUFFIE, D. e SINGLETON, K. Credit Risk: Pricing, Management and Meansurement,Princiton University Press, 2003.

[Egloff, 2004] EGLOFF, D. E VANINE, P., A simple Model of Credit Contagion. 2004 EFA Maastricht Meetings Paper, 1142.

[Erdos,1959] ERDÖS,P. and RéNYI, A., On random grafs I., Publicationes Matematticae ,6, 290-297, 1959

[Frey,2002] FREY, R. and McNEIL, J., VaR and Expected Shortfall in Portfolios of Dependent Credit Risks: Conceptual and Practical Insights, Jornal of Banking and Finance, 26, 1317-1334, January, 23, 2002

[Giesecke,2003] Giesecke, K., Credit Contagion and Aggregate Losses., Jornal of Economics Dynamics \& Control 30:2006, 741-767.

[Giesecke,2004] GIESECKE,K. e WEBER, S., Cyclical Correlations, Credit Contagion, and portfolio Losses, Jornal of Banking and Finance, November, 2003.

[Frank,1967] FRANK HARARY, F., Graph Theory and Theoritical Physics, Academic Press London and New York, 1967.

[Jarrow,1995] JARROW R.A. e TURNBULL, S.M. Pricing derivatives on financial securities subject to credit risk. J.Finance, 50: 53-85,1995.

[Jarrow,1997] JARROW R.A., LANDO, D. e TURNBULL, S.M. A Markov model for estructure of credit risk spreads. Rev. Finan. Stud., 10: 481-523, 1997. 
[Jarrow,2001] JARROW R.A., F. YU, Counterparty risk and pricing of defaultable securities, Journal of Finance 56: 1765-1800, 2001

[Lando, 1994] LANDO, D., On Cox processes and credit-risky securities. Rev. Derivatives Res., 2:99-120, 1998.

[Morgan,1996] J.P.MORGAN \& CO. INCORPORATED. CreditMetrics - Tecnical Document,New York: Riskmetrics Group, 1996.

[Molins,2004] MOLINS JORDI., VIVES EDUARD., Long rang Ising model for credit risk modeling in homogeneous portfolios physics.cond-mat (physics.stat-mech)V1, 21 Jan. 2004.

[Kaj,2003] KAJ NYSTROM AND JIMM SKOGLUND., Essentials of credit portfolio management Swedbank, Group Financial Risk Control, S-105 34 Stockholm, Sweden, March 20, 2003.

[Merton, 1974] MERTON, R. C. On the pricing of corporate debt: The risk structure of interest rates. J. Finance, 29: 449-470, 1974.

[Neu,2004] NEU P. e KUHN, R. Credit risk enhancement in a network of interdependent firms, Physica A 342: 639-655.

[Hull,1997] HULL JOHN, Options, futures and other derivatives, Third Edition, Prentice Hall, 1997.

[Hatchett,2006] HATCHETT JPL., E KUHN, R. Effects of Economic Interactions on risk, Journal of Finance, Januar 20: 1-24, 2006.

[Diestel,2000] DIESTEL REINHARD, Graph theory Graduate Texts in Mathematics, Springer-Verlag, 2000. 
[Ross,2002] ROSS, S., A First Course in Probability, sixth edition, Prentice Hall, 2002 .

[Shimko,1993] SHIMKO, D.,TEJIMA, N. AN DEVENTER, D.R. The pricing of risky debt when interest rates stocastic. J. Fixed Income,3(2): 58-65,1993.

[Schonbucher,2000] SCHONBUCHER, P.J. Credit Risk Modelling and Credit Derivatives. Tese de Doutorado, universidade de Bonn, 2000.

[Uzunov,1992] USUNOV,D. Theory of Critical Phenomena; Mean, Flutuations and Renormalization, Word Scientific Pub Co Inc, 1992.

[Vasicek, 1984] VASICEK, O. Credit Valuation KMV Corporation, 1984.

[Yu,2002] YOU, F., Default correlation in reduced-form models, Working paper, UC-irvine, 2002.

[Yu,2003] YOU, F., Dependent Default in Intensity-Based Models., EFA 2003 Annual Conference Paper No. 332.

[Zhou, 1996] ZHOU, C. A jumb-difusion approach to modelling credit risk and valuing defautable securities . Federal Reserves Board, Washington, 1996.

[Welsh,1993] WELSH, A., Complexity: Knots, colourings and Counting, London Mathematical Society, Lecture Note Series, 186, Cabridge University Press, 1993.

[Wilson,1997a] WILSON,T.,Credit Risk Modeling: A New Approach. Nova York: McKinsey INC., 1997 
[Wilson,1997b] WILSOn,T., Portfolio Credit Risk (Parts I e II.)Risk Magazine, 1997. 